AML - K - $77-3558-1$

\title{
Eould Lahoratories
}

\section{GOULD}




\section{DISCLAIMER}

This report was prepared as an account of work sponsored by an agency of the United States Government. Neither the United States Government nor any agency Thereof, nor any of their employees, makes any warranty, express or implied, or assumes any legal liability or responsibility for the accuracy, completeness, or usefulness of any information, apparatus, product, or process disclosed, or represents that its use would not infringe privately owned rights. Reference herein to any specific commercial product, process, or service by trade name, trademark, manufacturer, or otherwise does not necessarily constitute or imply its endorsement, recommendation, or favoring by the United States Government or any agency thereof. The views and opinions of authors expressed herein do not necessarily state or reflect those of the United States Government or any agency thereof. 


\section{DISCLAIMER}

Portions of this document may be illegible in electronic image products. Images are produced from the best available original document. 


\section{Prepared For:}

Argonne National Laboratory

9700 South Cass Avenue

Argonne, Illinois 60439

Released: 15 February 1977

(N)

DEVELOP NICKEL - ZINC

BATTERY SUITABLE FOR

ELECTRIC VEHICLE PROPULSION

Task A - Design and Cost Study

Contract No. 31-109-38-3558

Gould Report No. 762-003-1

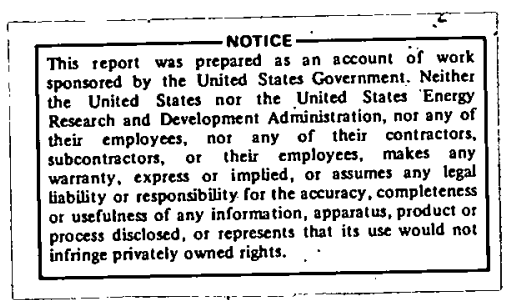

Gould Inc., New Business Division Nickel-Zinc Battery Project 30 Gould Center

Rolling Meadows, Illinois 60008 
I. BATTERY DESIGN AND PERFORMANCE 9

A. Analysis of Battery Configuration 9

B. Design Specifications of Proposed Batteries 11

C. Performance Specifications of Proposed Batteries 11

D. Analysis of Expected Performance in Vehicle Applications 11

II. CURRENT DEVELOPMENT PROBLEMS 58

A. Problems Related to System Performance Characteristics 58

1. Capacity Decline on Cycling 58

2. Electrolyte Maintenance 60

3. Thermal Characteristics 6

B. Component Problems 61

1. Separator Materials 61

2. Zinc Electrode $\quad 77$

3. Nickel Electrode 81

III. MANUFACTURING PROJECTIONS $\quad 83$

A. Projected Manufacturing Process 83

1. Electrode Manufacturing Processes 83

2. Separator Manufacturing Processes 91

3. Manufacturing Assembly Processes 91

4. Processes in Recycling of Used Batteries 94

B. Battery Manufacturing Cost Estimates 94

C. Capital Investment Estimates 98

D. Materials Requirements 98

E. Pilot Production Facility Layout 98

IV. PRELIMINARY TEST PROCEDURES, TEST STANDARDS, AND SAFETY

V. REFERENCES

APPENDIX A 


\section{LIST OF TABLES}

Page No.

Table I - 1 Gould Generation I

Electric Vehicle Cell Specifications

Table I - 2 Discharge Characteristics of Generation I

Electric Vehicle Nickel-Zinc Cell

Table I - 3 Low Energy Cell Description 15

Table I - 4 High Energy Cell Description 16

$\begin{array}{llll}\text { Table } 1 & -5 & \text { Design Specifications Of Proposed Cell } & 17\end{array}$

Table I $\quad-6 \quad$ Design Specifications Of Proposed 8-Cell Module 18

Table I - 7 Design Specifications Of Proposed 52-Volt Battery 19

Table I - - 8 Performance Specifications For Proposed Cell . 20

Table I - 9 Performance Specifications For Proposed Battery 21

Table I $\quad-10 \quad$ Comparative Battery Performance : 22

Table II $\quad-1 \quad$ Physical Properties Of Typical Separator Materials $\quad 76$

Table III - 1 Generation I Electric Vehicle Ni-Zn Cell 84

Table III - 2 Summary Of Manufacturing Cost Estimates . 99

Table III - 3 Manufacturing Cost Estimate $\quad 100$

Table III - 4 Manufacturing Cost Estimate . 101

Table III - 5 Manufacturing Cost Estimate 102.

$\begin{array}{llll}\text { Table III - 6a } & \text { Detailed Cost Breakdown } & 103\end{array}$

Table III - 6b Detailed Cost Breakdown 104

Table III - 6c Detailed Cost Breakdown . 105

Table III - 6d Detailed Cost Breakdown 106 
Table III - 7 Capital Investment Estimates

Table III $-8 \quad$ Capital Equipment Breakdown $\quad 108$

Table III - 9 Materials Requirement Per Year 109

Table III - $-10 \quad$ Battery Materials Requirement Vs. World Production $\quad 110$ 


\section{LIST OF ILLUSTRATIONS.}

Page No.

Figure I - 1 Typical Discharge Characteristics of Generation I

EV Ni-Zn Cell - Design A

Figure I - 2 Typical Discharge Characteristics of Generation I EV Ni-Zn Cell - Design B

Figure I - 3 Typical Discharge Characteristics of Generation I EV Ni-Zn Cell

Figure I - 4. Generation I Electric Vehicle Ni-Zn Cell -

Capacity As A Function Of Discharge Rate - Design A

Figure I - 5 Generation I Electric Vehicle Ni-Zn Cell -

Capacity As A Function Of Discharge Rate - Design B

Figure I - 6 Generation I Electric Vehicle Ni-Zn Cell

Comparison of Designs A and B

Figure I $\quad-7 \quad$ Specific Energy As a Function Of Capacity

Figure I $\quad-8$ Volumetric Energy As A Function Of Capacity 30

Figure I $\quad-9$ Battery Weight As A Function Of Capacity 31

Figure I $\quad-10 \quad$ Battery Volume As A Function Of Capacity . 32

Figure I $-11 \quad$ Battery Weight As A Function Of Capacity 33

Figure I $\quad-12$ Battery Volume As A Function Of Voltage 34

$\begin{array}{llll}\text { Figure I } & -13 & \text { Specific Energy As A Function Of Capacity } & 35\end{array}$

Figure I $-14 \quad$ Volumetric Energy As A Function Of Capacity 36

$\begin{array}{llll}\text { Figure I } & -15 & \text { Specific Energy As A Function Of Capacity } & 37\end{array}$

Figure I $\quad-16$ Volumetric Energy As A Function Of Capacity 38

Figure I -17 Specific Energy As A Function Of Discharge Rate 39

Figure I -18 Specific Energy As A Function Of Discharge Rate $\quad 40$

Figure I -19 Specific Energy As A Function Of Discharge Rate $\quad 41$ 
Figure I -20 Volumetric Energy As A Function Of Discharge Rate

Figure I $-21 \quad$ Volumetric Energy As A Function Of Discharge Rate

Figure I -22 Volumetric Energy As A Function Of Discharge Rate

Figure I -23 Specific Energy As A Function Of Cell Width

Figure I $-24 \quad$ Volumetric Energy As A Function Of Cell Width

Figure I $\quad-25$ Dimensions Of Proposed Cell

Figure I -26 Electrode Dimensions For Proposed Cell

Figure I . -27 Separator For Proposed Cell Configuration

Figure I $\quad-28$ Dimensions Of Proposed 8-Cell Battery Module

Figure I $\quad-29$ Dimensions Of Proposed 52-Volt Battery - Conf. A

Figure I - -30 Dimensions Of Proposed 52-Volt Battery - Conf. B.

Figure I -31 Dimensions Of Proposed 52-Volt Battery - Conf. C

Figure I - -32 Battery Voltage As A Function Of Charge Time At The 10 Hour Rate

Figure I -33 Charge - Discharge Efficiency As A Function Of Discharge Rate

Figure I $\quad-34 \quad$ Projected Cycle Life

Figure I $\quad-35$ Comparative Battery Performance

Figure II $\quad 1 \quad$ Cycle Life Of Generation I Cell

Figure II - 2 Cycle Life Of Ni- $\mathrm{Zn}$ Cells With Commercial Separators

Figure II - 3 Cycle Life Of Small Cells With Commercial Separators

Figure II - 4 Cycle Life Of Ni-Zn Cells With Commercial Separators

Figure II $\quad-5$ Cycle Life Of Generation I Cell With Nylon-Celgard Separation

Figure II - 6 Discharge Characteristics Of Generation I Cell With Nylon-Celgard Separation 
Figure II -7 Secondary Electron Images Of Cross-Sections Of Virgin Nylon Films

Figure II - 8 Secondary Electron Image Of Cross-Section Of Virgin Nylon Film

Figure II - 9 Secondary Electron Image Of Virgin Nylon Films

Figure II $\cdot 10 \mathrm{a}$ Secondary Electron Image Of Nylon Film After Cell Testing

Figure II -10b Elemental X-Ray Map of $\mathrm{Zn}$

Figure II -11a Secondary Electron Image Of Nylon Film After Cell Testing

Figure II -11b Elemental X-Ray Map of Zn

Figure II -11c Secondary Electron Image Of Nylon Film After Cell Testing

Figure II -12 Performance Of 40 Ah, 12-Volt Battery With Gould 1/O-Celgard Separation

Figure II $\quad-13 \quad 50$ Ah Cells

Figure II $-14 \quad 50$ Ah Cells With Original and Improved Zinc Electrodes

Figure III - 1 Manufacture Of NiO Electrode For Electric Vehicle Nickel-Zinc Battery - A.

Figure III - 2 Manufacture Of NiO Electrode For Electric Vehicle Nickel-Zinc Battery - B.

Figure III - 3 Manufacture Of NiO Electrode For Electric Vehicle Nickel-Zinc Battery - C.

Figure III -4 Manufacture Of NiO Electrode For Electric Vehicle Nickel-Zinc Battery - D.

Figure III - 5 Manufacture Of Zinc Electrode For Electric Vehicle Nickel-Zinc Battery - A.

Figure III - 6 Manufacture Of Zinc Electrode For Electric Vehicle Nickel-Zinc Battery - B. 
Figure III - 7. Manufacture Of Flexible Inorganic/Organic Composite Separator: $\quad 93$

Figure III - 8 Manufacturing Assembly Processes 95

Figure III - 9 Recycling Of Used Nickel-Zinc Batteries - A. 96

Figure III -10 Recycling Of Used Nickel-Zinc Batteries - B. 97

Figure III -11 Nickel-Zinc Battery Process Demonstration Facility 111 


\section{EXECUTIVE SUMMARY}

The following is a summary report of a three month design and cost study for the use of nickel-zinc batteries in electric vehicles. This document is not just the product of only three month's effort, but reflects the extensive experience of Gould in the following areas:

- 8 years direct experience in developing the nickel-zinc system costing Gould in excess of $\$ 1,000,000$.

- 20 years as one of the world's largest producers of nickel-cadmium and silverzinc batteries; these technologies and the manufacturing procedures are related to the nickel-zinc system.

- 8 years as the sole producer of high-quality nickel fuel cell electrodes (over 100,000 produced) for the Apollo lunar missions. Both the technology and manufacturing procedures are directly applicable to the nickel-zinc battery.

- 6 years as the largest supplier in the world of completely engineered propulsion systems (batteries, motors, and controllers) for on-road electric vehicles.

- 60 years as one of the world's major developer and manufacturer of both automobile and industrial lead-acid batteries. This commitment was responsible for the introduction of the maintenance-free battery.

- 60 years experience as one of the world's largest reclaimers of battery raw materials.

- 40 years as the largest U.S. powder metallurgy company. Both the nickel and zinc electrodes are powder metallurgy products. Mass production manufacturing procedures for the nickel-zinc battery are being developed from our OEM automotive experience.

- 16 years as one of the world's largest metal foil fabricators. These conductive foils are utilized for grid materials. This experience encompasses 
electroforming, perforating, and expanded metal forming techniques.

Because of the extensive present and prior commitment in both product and process development of nickel-zinc batteries, Gould's proprietary position is very strong and must be protected. As a result, this document was written in a manner not to divulge the key proprietary factors. However, if there is need for further amplification, Gould would be pleased to meet with the Project Officer on a confidential basis.

\section{DESIGN STUDY CONCLUSIONS}

\section{Verification of the State-of-the-Art}

The state-of-the-art is defined as cell or battery performance in tests representative of the electric vehicle application which is consistent with the design objectives provided by A.N.L. and has a high probability of being duplicated in volume production. This definition excludes, for example, the work by A.G.A. - Tudor in Sweden with vibrating electrodes because of its $\$ 100 / \mathrm{kWh}$ cost as estimated in Reference 1 .

In addition, promising component tests such as NASA's recent $1000+$ cycle separator tests (Reference 2) and Energy Research Corporation (ERC) tests (References 3 and 4) on nickel and zinc electrodes cannot be considered in a state-of-the-art analysis. NASA's tests were performed at shallow depth of discharge $(40-50 \%)$ and with cell sizes so small as to make impossible the prediction of large cell performance. For full discussion of the size effect, see Section II of the text. The ERC work on nickel electrodes, indicating 800 cycle capability, was performed in the nickel-hydrogen system. ERC even states in Reference 3 that performance of their non-sintered nickel electrode in the nickel-zinc system has a major problem with capacity loss after only 200 to 250 cycles. Similarly, ERC's zinc electrode work indicating 500 cycle performance was based upon 40 Ah cells, which is considered too small to extrapolate to the much larger electric vehicle size cells.

In an attempt to verify the state-of-the-art of nickel-zinc batteries for the electric vehicle application, Gould has conducted an extensive world-wide review for the last six months emphasizing personal contacts with industry, government, and academic leaders. The conclusion we have reached is that Gould's own 400 Ah cell tests, which have reached 270 cycles while maintaining $80 \%$ capacity, represents the known state-of-the-art. This performance, which is discussed in detail in Section I of this document, was limited by the chemical instability of the separator. With the solution of this problem, life will likely be limited to about 500 cycles 
by loss in capacity of the zinc electrodes due to shape change. Once this problem is resolved, life will then be limited by the sintered nickel electrode somewhere near 1000 cycles. This state-of-the-art analysis is depicted graphically in Exhibit 1. Included in this Exhibit is the rate of progress in separator development which leads to optimism in further improving the state-ofthe-art.

\section{EXHIBIT 1}

\section{FACTORS LIMITING NICKEL - ZINC PERFORMANCE AND RATE OF PROGRESS IN LMPROVING CYCLE LIFE}

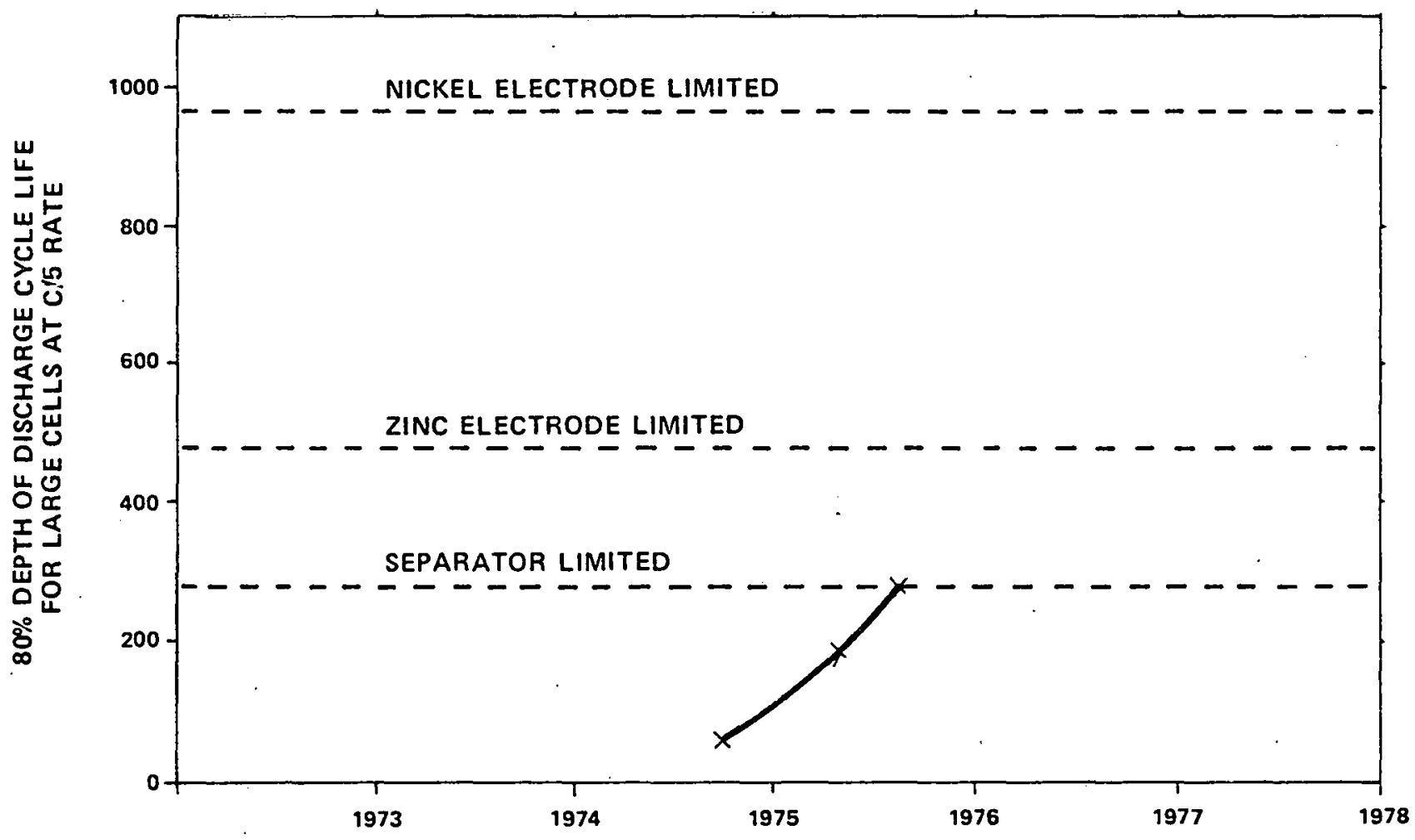

EXHIBIT 1: FACTORS LIMITING NICKEL-ZINC PERFORMANCE AND RATE OF PROGRESS IN IMPROVING CYCLE LIFE. 


\section{THREE YEAR RESEARCH \& DEVELOPMENT PLAN}

The proposed three year R\&D plan to achieve the target specific energy/power, cycle life, and cost goals is provided as a separate document, Vol II. Emphasis will be placed on the development of a separator to have less than 0.01 micron pore size, about a $10 \mathrm{ohm}-\mathrm{cm}$ resistivity, and with chemical stability for a 1000 cycle life. Section II, part B.1. of the text illustrates the promise that both organic and inorganic/organic concepts have for improving the state-of-the-art. It should be emphasized the work at Gould is only focused on materials and processes which have reasonable chances of meeting the cost target.

The next important $R \& D$ task will be work on minimizing zinc shape change. This effort will concentrate on further refining a proprietary geometric design which has pushed the current state-of-the-art to about 500 cycles. In addition, work on both organic and metallic additives to both reduce cost and to retain the shape of the zinc electrode is warranted. Preliminary work at Gould and at other companies has shown promise for this approach to push the state-of-the-art toward 1000 cycles without significantly increasing cost.

Work on the nickel electrode will not be emphasized until significant improvements in the separator and zinc electrodes are proven. Gould has had over 20 years of experience with its sintered nickel electrode and feels quite confident of its technological and economic superiority to the non-sintered version. The superior performance of the sintered version, especially at high discharge rates near the end of battery life, coupled with the possibility of reusing the electrode, are persuasive as long as its cost can be competitive. Our cost analysis, detailed in Section III B., shows that the $\$ 50 / \mathrm{kWh}$ target can be met with the sintered electrode approach even without an allowance for reusing or reclaiming the electrode materials. The sintering step itself, for example, will only cost approximately $\$ 0.10 /$ cell in large volume.

On the other hand, our analysis of the non-sintered approach is not as optimistic as that given in Reference 3 by ERC. Gould participated with ERC in 1972 in a program to evaluate the future production cost of their approach. The cost at that time was projected to be significantly more expensive than the sintered version. The need for a larger number of electrodes and grids to meet a specific capacity level is a major problem of the non-sintered approach. Furthermore, the recent cost analysis published by ERC (References 3 and 4) appears to be misleading. Their $\$ 10.60$ raw material projection for a 300 Ah cell equates to $\$ 0.022 / \mathrm{Wh}$. However, it must be noted that this figure represents "raw materials costs" and not the cost of materials which are required by the non-sintered process. For example, in Reference 3, nickel at $\$ 2.20 / \mathrm{Lb}$. is given 
as a raw materials cost, but it is known that nickel hydroxide must be used, with a known commercial cost in excess of $\$ 5.00 / \mathrm{Lb}$. Similarly, graphite is listed at $\$ 0.55 / \mathrm{Lb}$. when a powder form must be used and its commercial cost is at least $\$ 1.00 / \mathrm{Lb}$. The same is true for ERC's prices on steel, cobalt, and nickel plate, i.e., no conversion costs are allocated to put the various raw materials into a condition for use in the batteries. Thus, when comparing Gould's converted materials cost of $\$ 0.027 / \mathrm{Wh}$ (detailed in Section III B.) to ERC's cost of $\$ 0.022 / \mathrm{Wh}$, one is comparing apples and oranges. It is Gould's best estimate that the real materials cost for ERC is in excess of $\$ 0.03 / \mathrm{Wh}$ and, thus, the cost of materials for the non-sintered approach is probably more expensive than Gould's approach.

It is Gould's R \& D plan to not emphasize either the sintered or non-sintered nickel electrode approaches, but to concentrate on the much more important problems of separator and zinc electrode life.

\section{OPTIMUM CELL \& BATTERY MODULE SIZE}

Selection of optimum cell and battery size is complex and requires projections of the future vehicle systems designs. It is not sufficient to just consider the largest feasibie cell size, which would maximize energy density and minimize battery cost. A preliminary power system's tradeoff analysis is presented in Exhibit 2 which compares a 50-volt system, typical of the Gould/AM General postal vehicle, to that of a 100-volt system, assuming projected nickel-zinc battery costs.

EXHIBIT 2

\section{CELL SIZE TRADE-OFF ANALYSIS}

\begin{tabular}{|c|c|c|}
\hline & 50 Volt System: & 100 Volt System: \\
\hline Cell Size for $25 \mathrm{kWh}$ Application & $500 \mathrm{Ah}$ & $250 \mathrm{Ah}$ \\
\hline Installation Cost of Battery & $\$ 1,250$ & $\begin{array}{l}\$ 1,400 \\
(\$ 150 \text { extra per replacement })\end{array}$ \\
\hline Energy Density & $10 \%$ higher & Baseline \\
\hline Power System Efficiency & $\begin{array}{l}4 \% \text { lower than } \\
100 \text {-volt system }\end{array}$ & Baseline \\
\hline Power System Cost & $\begin{array}{l}\$ 100 \text { more than } \\
100 \text { - volt system }\end{array}$ & Baseline \\
\hline
\end{tabular}


The conclusion drawn from this analysis is that the lower cost (especially over lifetime when multiple battery replacements will occur) and high energy density of the 500 Ah cell is more attractive than the smaller capacity cell. This analysis will even further favor the larger capacity cell if power transistors successfully replace SCR's in controllers, which will reduce costs of controllers and be more compatible with low voltage systems. Recent private meetings with. both Cableform and Sevcon Ltd. in England, who are the largest electric vehicle controller manufacturers in Europe, have confirmed the above analysis of Gould and have unequivocally stated the availability of proven on-the-shelf controllers for this application.

The 500 Ah cell size Gould has selected as optimum from a preliminary design standpoint can be made to an almost infinite selection of dimensions and configurations. Cells selected for this study (see Figure I-25) weigh 25.1 pounds in a volume of 343 In. $^{3}$ with dimensions of 15.69 " high, 9.80 " long, and 2.23" wide. The full battery, for about $25 \mathrm{kWh}$ energy, will have 32 identical cells with a volume of $7.39 \mathrm{Ft}^{3}$ and 818 pound weight or a $32 \mathrm{Wh} / \mathrm{Lb}$. energy density. In this same volume and weight, a battery with an energy density of about $42 \mathrm{Wh} / \mathrm{Lb}$. is projected within the next three years.

Two versions of this cell can be considered, i.e., low and high energy designs. The low energy design is based upon concepts already well proven in large cells, while the high energy concept is less fully developed. The performance targets of these cells is summarized in Exhibit 3.

\section{EXHIBIT 3}

500 Ah CELL PERFORMANCE OBJECTIVES

\begin{tabular}{|c|c|c|c|c|}
\hline \multirow[b]{3}{*}{ Discharge Rate } & \multicolumn{2}{|c|}{ Low Energy Cell } & \multicolumn{2}{|c|}{ High Energy Cell } \\
\hline & (\% of Rated & & (\% of Rated) & \\
\hline & Capacity & Wh/Lb. & Capacity & Wh/Lb. \\
\hline $\mathrm{C} / 5$ & 100.0 & 32.2 & 100.0 & 42.0 \\
\hline $\mathrm{C} / 3$ & 99.9 & 31.4 & 99.0 & 40.2 \\
\hline $\mathrm{C} / 1$ & 94.0 & 25.9 & 86.5 & 30.0 \\
\hline
\end{tabular}


Results on 12 volt batteries tested by Gould indicated that constant current charging at the $\mathrm{C} / 10$ rate with a voltage limit will provide a suitable recharge. Most importantly, a reasonably simple charger design utilizing constant potential techniques appears possible because of the slope near the end of charging as shown in the text in Figure 1-32. Charge-discharge efficiency for 12-volt batteries has been slightly in excess of $90 \%$. This extremely good efficiency appears related to the superior conductivity of Gould's sintered nickel electrode design. For comparison, recent data by General Motors (Reference 5) indicates only about an $80 \%$ charge-discharge efficiency for their non-sintered electrode approach.

\section{MANUFACTURING COSTS \& CAPITAL INVESTMENT}

Projected manufacturing procedures for producing Gould's nickel-zinc battery are outlined in Section III of the text. The pilot plant needed to verify the product quality and volume manufacturing cost is also detailed. Of importance is that Gould has already started the first phase of the pilot plant by establishing a manufacturing facility for the electrode fabrication steps. Exhibit 4 summarizes manufacturing cost estimates and capital investment requirements as a function of volume.

\section{EXHIBIT 4}

PROJECTED MANUFACTURING COSTS \& CAPITAL INVESTMENTS FOR 500 Ah CELLS AS A FUNCTION OF ANNUAL VOLUME

\begin{tabular}{|c|c|c|c|c|c|}
\hline $\begin{array}{c}\text { Volume } \\
\text { Batteries/Yr. }\end{array}$ & $\begin{array}{c}\text { Capital } \\
\text { Investment } \\
\text { ( } \$ \text { million }) \\
\end{array}$ & $\begin{array}{c}\text { Labor \& } \\
\text { Conversion } \\
\text { Cost } / \text { Cell } \\
(\$) \\
\end{array}$ & $\begin{array}{c}\text { Material } \\
\text { Cost/Cell } \\
(\$) \\
\end{array}$ & $\begin{array}{c}\text { Total } \\
\text { Cost/Cell } \\
(\$) \\
\end{array}$ & $\begin{array}{c}\text { Total } \\
\text { Cost/kWh } \\
(\$) \\
\end{array}$ \\
\hline \multicolumn{6}{|l|}{ Pilot } \\
\hline 1,304 & 1.755 & 21.14 & 26.72 & 47.86 & 58.72 \\
\hline 6,520 & 2.554 & 11.08 & 26.18 & 37.26 & 45.71 \\
\hline \multicolumn{6}{|l|}{ Production } \\
\hline 13,040 & 5.320 & 6.24 & 21.64 & 27.88 & 34.20 \\
\hline 130,400 & not estimated & 6.00 & 21.64 & 27.64 & 33.91 \\
\hline
\end{tabular}


The pilot plant and first production stages have been studied in great detail. A general contractor and subcontractors have been consulted to develop sophisticated waste treatment techniques which would make the plants totally nonpolluting and, thus, have no adverse environmental impact. Approximately 12-18 months is required for each stage of the plant and equipment purchase and installation.

Raw materials to be utilized by each phase of production is given in the text in Table III-9. At this time, Gould does not believe that raw material supply will be a problem even at very large volume. Confidence for this statement comes from Gould's experience with lead recycling for lead-acid batteries. Today, about $93 \%$ of the lead is recovered which leads to a stable secondary lead market and helps conserve the supply of raw materials. Gould feels that significant use of nickel-zinc batteries will encourage a system for reusing nickel and zinc. This will lead to a lower cost $/ \mathrm{kWh}$ than that projected in Exhibit 4 and reduce cost pressures on raw materials. 


\section{BATTERY DESIGN AND PERFORMANCE}

\section{A. Analysis of Battery Configuration}

The optimum configuration and capacity size of a battery for electric vehicle applications cannot be defined without the input of electric vehicle designers. Futhermore, battery configuration and capacity size may vary with the type of vehicle and its intended mission. The intent of this analysis is to discuss characteristics of a number of batteries, each approximating $25 \mathrm{kWh}$ and ranging from 36 to 120 volts. This results in cells ranging in capacity from about $200 \mathrm{Ah}$ for the 120 volt batteries to about $700 \mathrm{Ah}$ for the 36 volt batteries. It is hoped that this preliminary data will provide general guidelines for the electric vehicle designers and give some direction on the preferred battery configuration (s) from the manufacturer's technical and economic point of view.

Predicted values of the various size cells given in this analysis are based on measured performance data from the 400 Ah size cell under development at Gould. Battery characteristics are shown at two levels - one for present state-of-the-art based on measured performance data from Gould's $400 \mathrm{Ah}$ size cell (low energy cell) and the other projected on the basis of limited, but demonstrated, performance data from Gould's more advanced 400 Ah cell (high energy cell). The specifications and performance characteristics of the present Gould $400 \mathrm{Ah}$ (Generation I Electric Vehicle) cells are shown in Tables $\mathrm{I}-1$ and $\mathrm{I}-2$ and Figures $\mathrm{I}-\mathrm{I}$ through $\mathrm{I}-6$. The performance is given for two configurations, designated as Design $A$ and Design B. The only differences in the two configurations is the number of electrodes - Design A contains $8 \mathrm{Ni}$ and $9 \mathrm{Zn}$, while Design $\mathrm{B}$ has $10 \mathrm{Ni}$ and $11 \mathrm{Zn}$.

Data on our analysis aimed at optimizing the battery configuration are summarized in Tables I-3 and I-4 and Figures I-7 through I-24. Within the parameters covered by this analysis, the following key deductions are reached:

- Energy density increases with cell capacity. For example, a $695 \mathrm{Ah}, 36$ volt (25 $\mathrm{kWh}$ ) battery can be as much as $31 \%$ and $35 \%$ higher in specific energy $(\mathrm{Wh} / \mathrm{lb})$ and volumetric energy $\left(\mathrm{Wh} / \mathrm{in}^{3}\right)$ than a $208 \mathrm{Ah}, 120$ volt $(25 \mathrm{kWh})$ battery (see Figures I-7 through I-8, respectively).

- Likewise, the weight and volume for a $25 \mathrm{kWh}$ battery decreases with increasing capacity (Figures $I-9$ and $\mathrm{I}-10$ ), or decreases with decreasing voltage (Figures I-11 and $\mathrm{I}-12$ ). 
- Increasing cell capacity increases energy density irrespective of the discharge rate (Figures I-13 through I-16, and I-17 through I-22).

- For each battery voltage group analyzed (e.g., 36, 48, 72, 96, and 120 volts) the cell with the thinnest width occupies the smallest space (area) and volume and yields the highest energy densities (Figures I-23 and I-24).

- Increasing the length and height of the cell decreases the $25 \mathrm{kWh}$ battery weight and volume more significantly than increasing the cell width (Figures I-11 and I-12).

From a battery cost standpoint, higher capacity, lower voltage designs are preferred. However, in the consideration of the total system performance, the electric vehicle designer may find it more efficient to go the oppoșite route. Thus, more interface between the battery developers and the electric vehicle designers is needed before drawing conclusions on an optimum capacity size. With regard to heat dissipation, cell configurations having thinner widths would seem more desirable. Decreasing the width of the cell (e.g., decreasing the number of electrodes) also results in higher utilization of cell volume as electrodes (e.g., decreased layers of separation).

With batteries in the 20 to $30 \mathrm{kWh}$ range, analysis of the various cell configurations suggests that a 36 -volt, 700 Ah battery could optimize energy density. However, a 50 -volt system is generally considered the lower limit by most electric vehicle designers. On this basis, a preferred cell configuration suitable for a 50 -volt system is:

$$
\begin{array}{llr}
\text { Height } & \ldots & 15.69 \text { in. } \\
\text { Length } & \ldots & 9.80 \text { in. } \\
\text { Width } & \ldots & 2.23 \text { in. }
\end{array}
$$

A battery consisting of 31 such cells would have a capacity of 500 Ah and $25.3 \mathrm{kWh}$ at 50.5 volts for the present state-of-the-art (based on measured performance of Gould's 400 Ah cell), i.e., low energy cell design. For the high energy cell design, a capacity of $658 \mathrm{Ah}$ is projected in this cell configuration (equivalent to $33 \mathrm{kWh}$ at 50.5 volts). 


\section{B. Design Specifications of Proposed Battery}

The design specifications for the suggested cell configuration are summarized in Table I-5. Drawings of the dimensions of the cell, electrodes and separator are shown in Figures I-25 through I-27.

The proposed battery configurations are based on 8-cell modules. The design specifications for the battery module are summarized in Table I-6 and illustrated in Figure I-28.

Four modules can be arranged in three different ways to provide a 52-volt battery. These are sketched in Figures I-29 through I-31. Table I-7 summarizes the design specification of the proposed 52-volt battery.

\section{Performance Specifications of Proposed Battery}

Performance data for the proposed cells and 52-volt batteries of low and high energy designs are given in Tables I-8 and I-9, respectively.

The battery charge voltage profile is shown in Figure 1-32 for the 52-volt battery. The data is based on measured performance of an 8-cell battery. The voltage rise at the end of the charge suggests that constant potential charging methods may be possible. This method would be preferred over a constant current charge from a cost standpoint as well as technical (e.g., easier control of overcharge). Furthermore, at higher charge rates, the voltage rise at end of charge would be more pronounced, thus making high rate charging more feasible.

Figure I-33 illustrates the charge - discharge efficiency of our nickel-zinc battery. Efficiencies up to $90 \%$ have been demonstrated and further improvements are probable.

Based on data presented elsewhere in this report, the projected cycle life shown in Figure I-34 is, in our opinion, achievable within the next three years.

\section{Analysis of Expected Performance in Vehicle Applications}

Driving range as a function of battery weight was estimated (Figure 1-35) using the performance characteristics computed for the high energy cell ( $43 \mathrm{Wh} / \mathrm{Lb}$.). The SAE Metropolitan Area Driving Cycle was used for calculating the range. This cycle implies an average speed of $24 \mathrm{mph}$, and one stop per mile. Thus, for a vehicle weighing approximately 3,240 lb. (including $450 \mathrm{lb}$. payload), the proposed 52-volt battery with an energy of $34 \mathrm{kWh}(815 \mathrm{lb}$.) would propel this vehicle for about 105 miles, compared to about 28 miles (26\%) for a $13 \mathrm{Wh} / \mathrm{Lb}$. (6 hour rate) lead-acid battery. 
The range on level ground at a steady speed of $30 \mathrm{mph}$ would be about 270 miles. A smaller battery (e.g., $\sim 25 \mathrm{kWh}, 580 \mathrm{Lb}$.) at the same specific energy $(43 \mathrm{Wh} / \mathrm{Lb}$.) would have a range of about 75 miles under the SAE driving cycle. In contrast, the lead-acid battery at $13 \mathrm{Wh} / \mathrm{Lb}$. (6 hour rate) and weighing $580 \mathrm{lb}$. would be limited to a range of about 16 miles ( $21 \%$ of that delivered by the $\mathrm{Ni}-\mathrm{Zn}$ battery).

The expected performance in the electric postal vehicle, compared to the commercial lead-acid battery, is tabulated in Table I-10 for batteries of equal weights $(1,300 \mathrm{lb}$.). As can be seen, the high energy Ni-Zn battery would provide a range of up to 66,600 miles at a cycle life of 500 (134 miles/cycle), compared to 18,000 miles at 600 cycles $(30 \mathrm{miles} / \mathrm{cycle}$ ) for the commerical lead-acid battery. These ranges, at the specified number of cycles, assume essentially full discharges each cycle. In the case of the $\mathrm{Ni}-\mathrm{Zn}$ system, however, the depth-of-discharge would be considerably less than $100 \%$ on the average in this application. At $50 \%$ DOD or about 67 miles per mission, the number of cycles would be doubled $(1,000)$. 


\section{GOULD GENERATION I \\ ELECTRIC VEHICLE CELL SPECIFICATIONS}

NOMINAL VOLTAGE

1.63

CAPACITY AT 6 HOURS

Ah

400

Wh

650

ENERGY DENSITY, Wh/Lb AT 6 HOURS

$30 \cdot 35$

ENERGY/UNIT VOLUME, WH/In. ${ }^{3}$ AT 6 HOURS

$\sim 2.2$

WEIGHT, POUNDS

$19 \cdot 21$

ELECTRODE SIZE

POSITIVE

$11.7 \ln . \mathrm{H} \times 05.5 \ln . \mathrm{L}$

NEGATIVE

$12.0 \ln . H \times 05.8 \ln . \mathrm{L}$

No. ELECTRODES/CELL

POSITIVE

8 or 10

NEGATIVE

9 or 11

OVERALL DIMENSIONS INCLUDING TERMINALS

CELL DIMENSIONS

$15.39 \mathrm{In} . \mathrm{H} \times 06.70 \mathrm{ln} . \mathrm{L} \times 02.95 \mathrm{In} . \mathrm{W}$

$14.27 \mathrm{In} . \mathrm{H} \times 06.70 \mathrm{In} . \mathrm{L} \times 02.95 \mathrm{In} . \mathrm{W}$ 
TABLE $\quad 1-2$

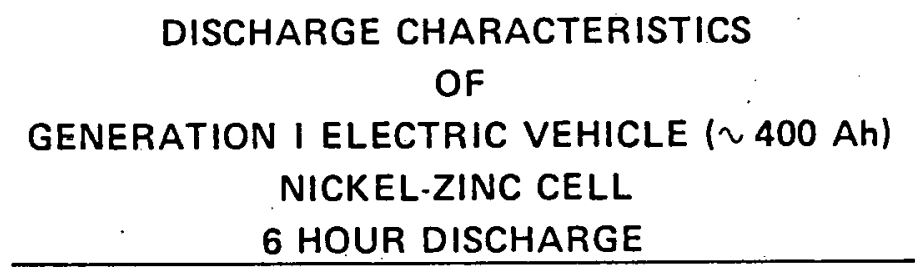

DISCHARGE, $\%$ OF RATED CAPACITY

DISCHARGE VOLTAGES FOR VARIOUS CYCLES

5
20
40
60
80

20

100

250

1.77

1.77

1.75

1.66

1.67

1.65

1.65

1.65

1.60

1.63

1,62

1.59

1.60

1.58

1.51 
TABLE $\quad 1.3$

LOW ENERGY CELL DESCRIPTION

(BASED ON MEASURED PERFORMANCE OF GOULD 400 Ah CELL)

CELL CAPACITY

(Ah at 6 HOUR RATE)

210
210
210

261

262

253

348

346

338

$415^{*}$

520

521

548

700

697

675
11.09

15.39

15.39

13.39

15.39

15.39

15.39

17.89

15.39

15.39

22.59

15.39

15.39
CELL DIMENSIONS (In.)

LENGTH WIDTH

6.70

2.950

2.950

2.950

6.70

6.70

4.85

6.70

2.950

2.950

1.991

6.70

2.950

5.80

2.950

6.70

2.471

6.70

2.950

6.70

7.80

2.950

$6.70 \quad 3.695$

6.70

2.950

9.80

2.950

6.70

4.388

\footnotetext{
* Gould 400 Ah Cell
} 
HIGH ENERGY CELL DESCRIPTION

(BASED ON LIMITED MEASURED PERFORMANCE OF GOULD 400 Ah CELL)

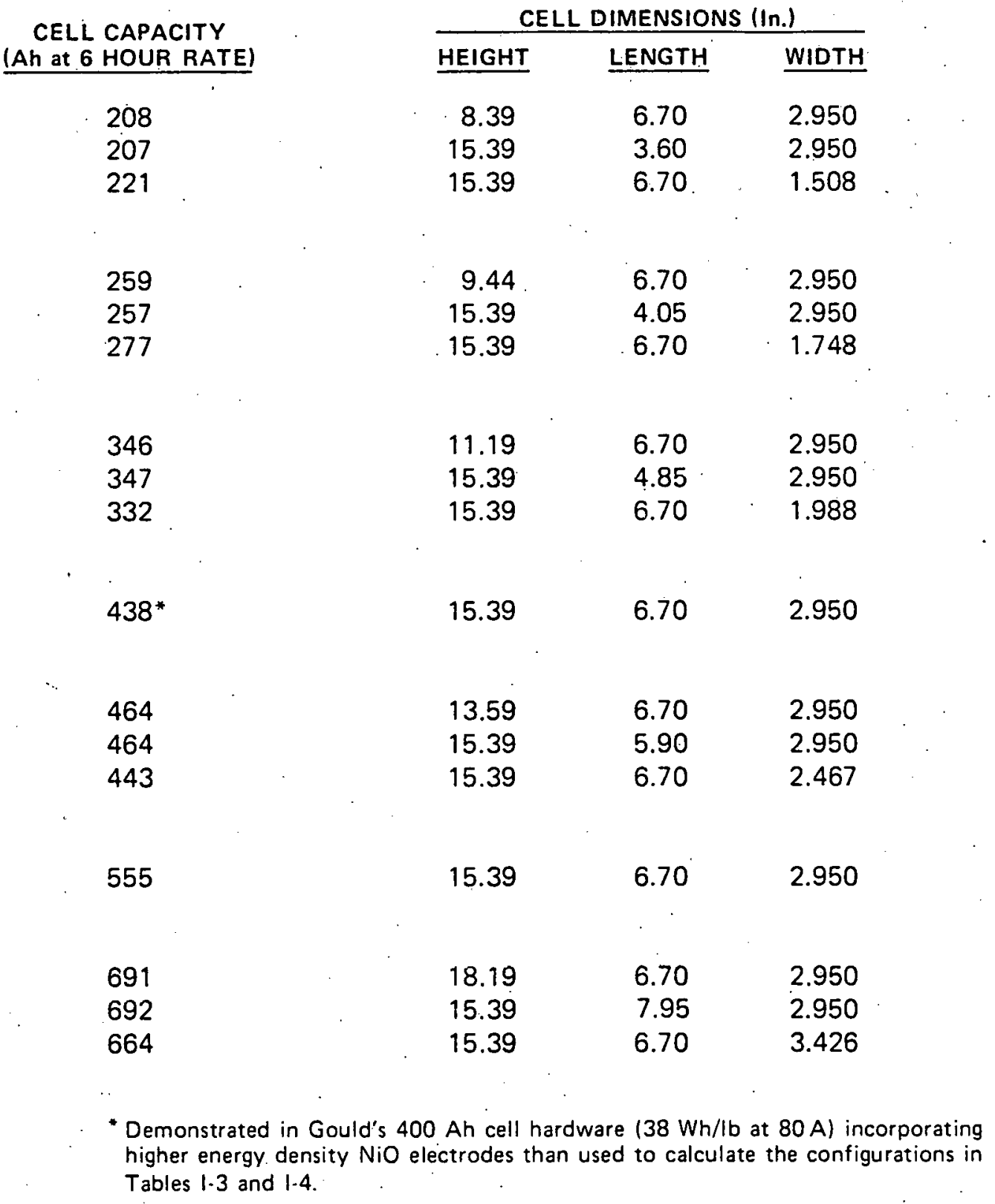


TABLE 1.5

\section{DESIGN SPECIFICATIONS OF PROPOSED CELL}

CAPACITY AT 6 HOUR RATE:

Ah

VOLUME (In. $\left.{ }^{3}\right)$

WEIGHT (Lb.)

ELECTRODE SIZE (In.):

POSITIVE

NEGATIVE (INNER)

NEGATIVE (OUTER)

No. ELECTRODES PER CELL:

POSITIVE

NEGATIVE (INNER)

NEGATIVE (OUTER)

OVERALL DIMENSIONS (In.)

SEPARATOR:

DIMENSIONS (In./LAYER)

No. LAYERS PER CELL

TERMINAL POSTS DIAMETER (|n.)

(Ni Plated $\mathrm{Cu}$ )
LOW ENERGY CELL
500

815

343

25.1
HIGH ENERGY CELL

$12.0 \mathrm{H} \times 8.60 \mathrm{~L} \times 0.095 \mathrm{~W}$ $12.3 \mathrm{H} \times 8.90 \mathrm{~L} \times 0.095 \mathrm{~W}$ $12.3 \mathrm{H} \times 8.90 \mathrm{~L} \times 0.054 \mathrm{~W}$

$12.0 \mathrm{H} \times 8.60 \mathrm{~L} \times 0.130 \mathrm{~W}$ $12.3 \mathrm{H} \times 8.90 \mathrm{~L} \times 0.060 \mathrm{~W}$ $12.3 \mathrm{H} \times 8.90 \mathrm{~L} \times 0.035 \mathrm{~W}$
658

1066

343

25.0 
TABLE $1-6$

DESIGN SPECIFICATIONS OF PROPOSED 8-CELL MODULE

LOW ENERGY CELL

CAPACITY AT 6 HOUR RATE:

Ah

$k W h$

No. OF CELLS

VOLTAGE

DIMENSIONS (ln.):

8.CELL OVERALL

VOLUME (Ft. $\left.{ }^{3}\right)$ :

8.CELL

OVERALL

WEIGHT (Lb.):

8.CELL

OVERALL
$15.69 \mathrm{H} \times 17.85 \mathrm{~L} \times 9.80 \mathrm{~W}$ $15.69 \mathrm{H} \times 18.85 \mathrm{~L} \times 10.80 \mathrm{~W}$

1.59

1.85

1.59

1.85

201

205
HIGH ENERGY CELL

$$
658
$$

8.53

8

13 $15.69 \mathrm{H} \times 18.85 \mathrm{~L} \times 10.80 \mathrm{~W}$ 
TABLE $\quad 1.7$

\section{DESIGN SPECIFICATIONS OF PROPOSED 52 - VOLT BATTERY}

LOW ENERGY CELL

CAPACITY AT 6 HOUR RATE:

$$
\begin{aligned}
& A h \\
& k W h
\end{aligned}
$$

NO. OF MODULES

No. OF CELLS

VOLTAGE

OVERALL DIMENSIONS (In.):

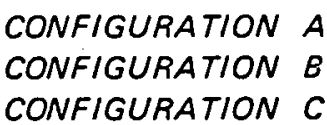

VOLUME (Ft. $\left.{ }^{3}\right)$

WEIGHT (Lb.)
500

26.1

4

32

52.16

$15.69 \mathrm{H} \times 75.39 \mathrm{~L} \times 10.80 \mathrm{~W}$ $15.69 \mathrm{H} \times 43.20 \mathrm{~L} \times 18.85 \mathrm{~W}$

$15.69 \mathrm{H} \times 37.70 \mathrm{~L} \times 21.60 \mathrm{~W}$

7.39

818
HIGH ENERGY CELL

\section{8}

34.1

4

32

51.84
$15.69 \mathrm{H} \times 75.39 \mathrm{~L} \times 10.80 \mathrm{~W}$ $15.69 \mathrm{H} \times 43.20 \mathrm{~L} \times 18.85 \mathrm{~W}$ $15.69 \mathrm{H} \times 37.70 \mathrm{~L} \times 21.60 \mathrm{~W}$

7.39

815 
TABLE 1.8

\section{PERFORMANCE SPECIFICATIONS FOR PROPOSED CELL}

\section{(1.15 VOLT CUT.OFF)}

\section{CELL ENERGY}

LOW ENERGY CELLS

\begin{tabular}{|c|c|c|c|c|}
\hline & & \multirow{3}{*}{$\begin{array}{c}\text { MID } \\
\text { VOLTAGE }\end{array}$} & \multirow{2}{*}{\multicolumn{2}{|c|}{ CAPACITY }} \\
\hline \multirow[b]{2}{*}{ Hr. RATE } & \multirow[b]{2}{*}{ AMPS } & & & \\
\hline & & & Ah & Wh \\
\hline 6 & 80 & 1.63 & 501 & 817 \\
\hline 5 & 100 & 1.615 & 500 & 808 \\
\hline 3 & 165 & 1.575 & 500 & 788 \\
\hline 1 & 500 & 1.38 & 471 & 650 \\
\hline
\end{tabular}

HIGH ENERGY CELL

\begin{tabular}{|c|c|c|c|}
\hline \multirow[b]{2}{*}{ AMPS } & \multirow{2}{*}{$\begin{array}{c}\text { MID } \\
\text { VOLTAGE }\end{array}$} & \multicolumn{2}{|c|}{ CAPACITY } \\
\hline & & $A h$ & Wh \\
\hline 110 & 1.62 & 658 & 1066 \\
\hline 130 & 1.595 & 658 & 1050 \\
\hline 220 & 1.545 & 651 & 1006 \\
\hline 650 & 1.32 & 569 & 751 \\
\hline
\end{tabular}

\section{ENERGY DENSITY}

\begin{tabular}{|c|c|c|}
\hline \multirow[b]{2}{*}{ Hr. RATE } & \multicolumn{2}{|c|}{ LOW ENERGY CELLS } \\
\hline & Wh/Lb. & $W h / \ln .^{3}$ \\
\hline 6 & 32.5 & 2.38 \\
\hline 5 & 32.2 & 2.36 \\
\hline 3 & 31.4 & 2.30 \\
\hline 1 & 25.9 & 1.90 \\
\hline
\end{tabular}

\begin{tabular}{cc}
\multicolumn{2}{c}{ HIGH ENERGY CELLS } \\
\hline Wh/Lb. & $\frac{\text { Wh/ln. }}{3}$ \\
\hline 42.6 & 3.11 \\
42.0 & 3.06 \\
40.2 & 2.93 \\
30.0 & 2.19
\end{tabular}

SPECIFIC POWER (1)

\section{Hr. RATE.}

6
5
3
1
0.5
0.33

(1) Based on Midpoint Voltages at the Specified Rates. (2) Estimated

\section{LOW ENERGY CELLS}

\begin{tabular}{|c|c|}
\hline AMPS & W/Lb. \\
\hline 80 & 5.2 \\
\hline 100 & 6.4 \\
\hline 165 & 10.4 \\
\hline 500 & 27.5 \\
\hline 1000 & $49.0^{(2)}$ \\
\hline 1500 & $69.3^{(2)}$ \\
\hline
\end{tabular}

\section{HIGH ENERGY CELLS}

\begin{tabular}{cc} 
AMPS & W/Lb. \\
\cline { 3 - 3 } 110 & 7.1 \\
130 & 8.3 \\
220 & 13.6 \\
650 & 34.3 \\
1300 & $61.4^{(2)}$ \\
1950 & $89.7^{(2)}$
\end{tabular}


TABLE 1.9

\section{PERFORMANCE SPECIFICATIONS FOR PROPOSED BATTERY}

(1.15 VOLT / CELL CUT.OFF)

ENERGY

LOW ENERGY CELLS

\begin{tabular}{|c|c|c|c|c|}
\hline \multirow[b]{2}{*}{ Hr. RATE } & \multirow[b]{2}{*}{ AMPS } & \multirow{2}{*}{$\begin{array}{c}\text { MID } \\
\text { VOLTAGE } \\
\end{array}$} & \multicolumn{2}{|c|}{ CAPACITY } \\
\hline & & & Ah & kWh \\
\hline 6 & 80 & 52.16 & 501 & 26.13 \\
\hline 5 & 100 & 51.68 & 500 & 25.84 \\
\hline 3 & 165 & 50.40 & 500 & 25.20 \\
\hline 1 & 500 & 44.16 & 471 & 20.80 \\
\hline
\end{tabular}

HIGH ENERGY CELLS

\begin{tabular}{|c|c|c|c|}
\hline \multirow[b]{2}{*}{ AMPS } & \multirow{2}{*}{$\begin{array}{c}\text { MID } \\
\text { VOLTAGE } \\
\end{array}$} & \multicolumn{2}{|c|}{ CAPACITY } \\
\hline & & Ah & $\mathrm{kWh}$ \\
\hline .110 & 51.84 & 658 & 34.11 \\
\hline 130 & 51.04 & 658 & 33.58 \\
\hline 220 & 49.44 & 651 & 32.19 \\
\hline 650 & 42.24 & 569 & 24.03 \\
\hline
\end{tabular}

ENERGY DENSITY

\section{LOW ENERGY CELLS}

Hr. RATE

6

5

3

1
Wh/Lb.

31.9

31.6

30.8

25.4
Wh/ln. ${ }^{3}$

2.05

2.02

1.97

1.63
HIGH ENERGY CELLS

Wh/Lb.

Wh/ln. ${ }^{3}$

41.9

41.2

2.63

39.5

2.52

29.5

SPECIFIC POWER ${ }^{(1)}$

\begin{tabular}{c} 
Hr. RATE \\
\hline 6 \\
5 \\
3 \\
1 \\
0.5 \\
0.33
\end{tabular}

6

5

3

0.5

0.33
LOW ENERGY CELLS

\begin{tabular}{c} 
AMPS \\
\hline 80 \\
100 \\
165 \\
500 \\
$1000^{(2)}$ \\
$1500^{(2)}$
\end{tabular}

W/Lb.

5.1

6.3

10.0

27.0

48.1

68.1
HIGH ENERGY CELLS

$\begin{array}{cr}\text { AMPS } & \text { W/Lb. } \\ 110 & 7.0 \\ 130 & 8.1 \\ 220 & 13.3 \\ 650 & 33.7 \\ 1300^{(2)} & 60.2 \\ 1950^{(2)} & 88.0\end{array}$

(1) Based on Midpoint Voltages at the Specified Rates.

(2) Estimated 


\section{COMPARATIVE BATTERY PERFORMANCE}

\section{PROJECTED PERFORMANCE IN POST OFFICE VEHICLE EQUIVALENT WEIGHT BATTERIES}

\section{CYCLE LIFE}

BATTERY Wt., Lb.

CAPACITY, kWh@ $125 \mathrm{~A}$

BATTERY VOLTAGE @ 125 A

RELATIVE USEFUL RANGE

RELATIVE USEFUL LIFE

(Cycles $x$ Useful Range Factor)

POST OFFICE MISSION

(Mileage at 30 Miles Per Mission)

\begin{tabular}{|c|c|}
\hline $\begin{array}{c}\text { CURRENT } \\
\text { LEAD.ACID(1) }\end{array}$ & $\begin{array}{c}1979 \\
\text { NICKEL-ZINC (2) } \\
\end{array}$ \\
\hline $600^{(3)}$ & $500^{(4)}$ \\
\hline 1,300 & 1,300 \\
\hline 12.6 & 55.9 \\
\hline 50.4 & 52.2 \\
\hline 1.0 & 4.44 \\
\hline 600 & 2,220 \\
\hline 18,000 & $\quad 66,600$ \\
\hline
\end{tabular}

(1) Gould E.V. Battery, IBD 66-E11.

(2) High Energy Cell at 43 Wh/Lb.

(3) Projected

(4) Minimum Goal 
\% OF RATED CAPACITY

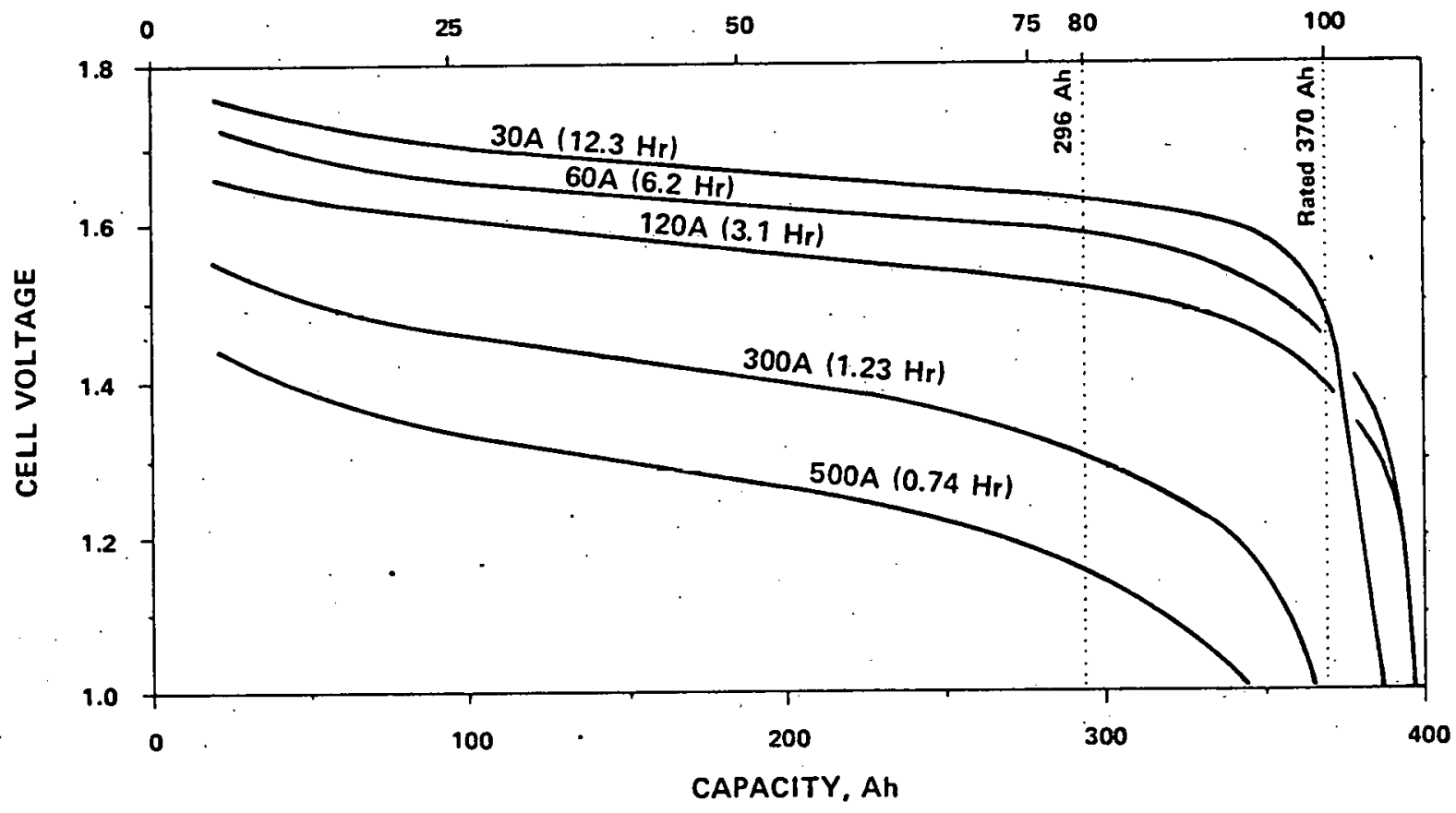

FIGURE 1-1:

TYPICAL DISCHARGE CHARACTERISTICS OF GENERATION I EV Ni-Zn CELL. Design A / Rated Capacity - 370 Ah at 3 to 6 Hour Rates (95\% of Actual 390 Ah to 1.3 V). 
$\%$ OF RATED CAPACITY

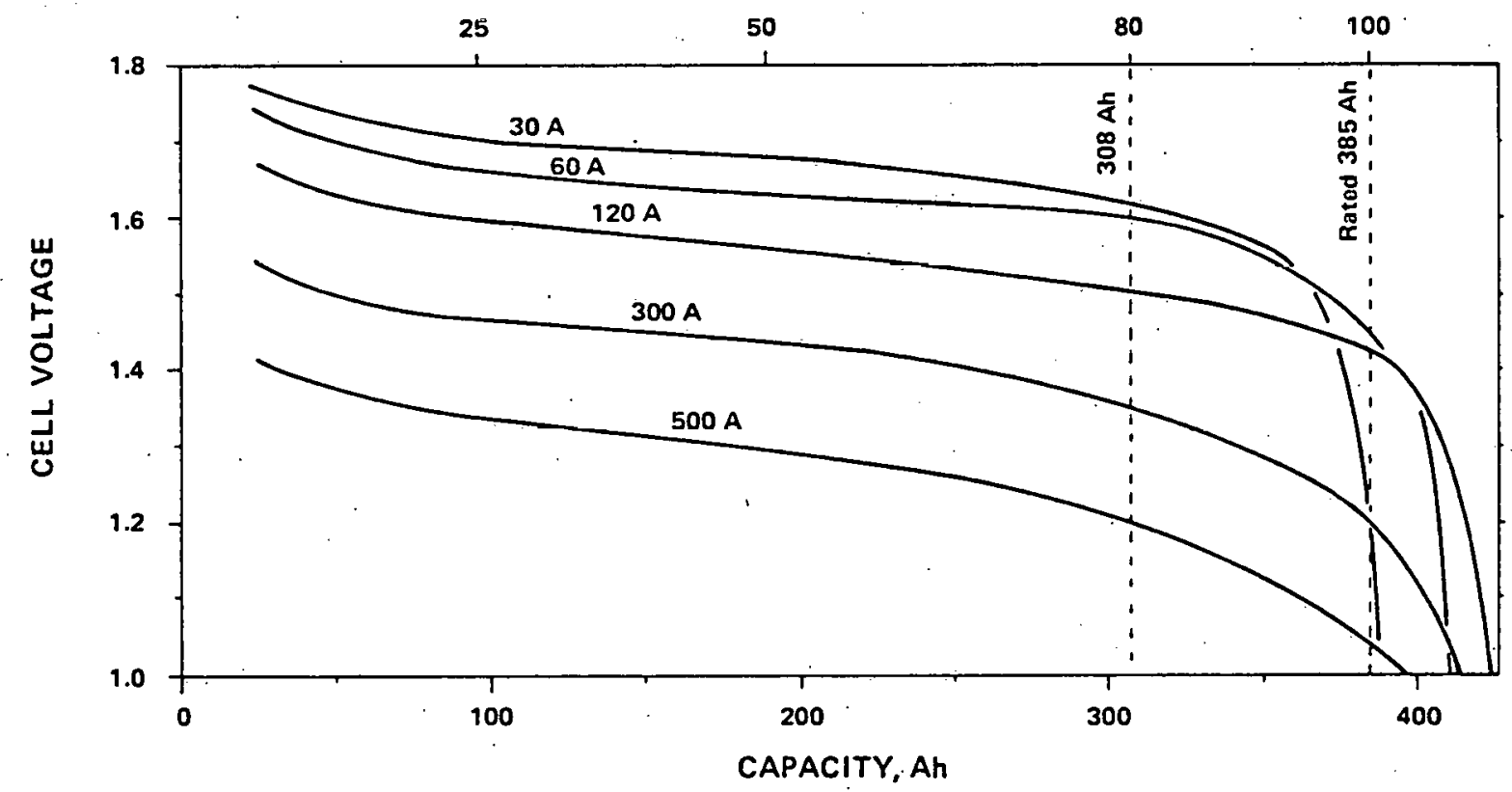

FIGURE 1-2:

TYPICAL DISCHARGE CHARACTERISTICS OF GENERATION I EV Ni-Zn CELL.

Design B / Rated Capacity - 385 Ah at 3 to 6 Hour Rates (95\% of Actual 406 Ah to 1.3 V). 


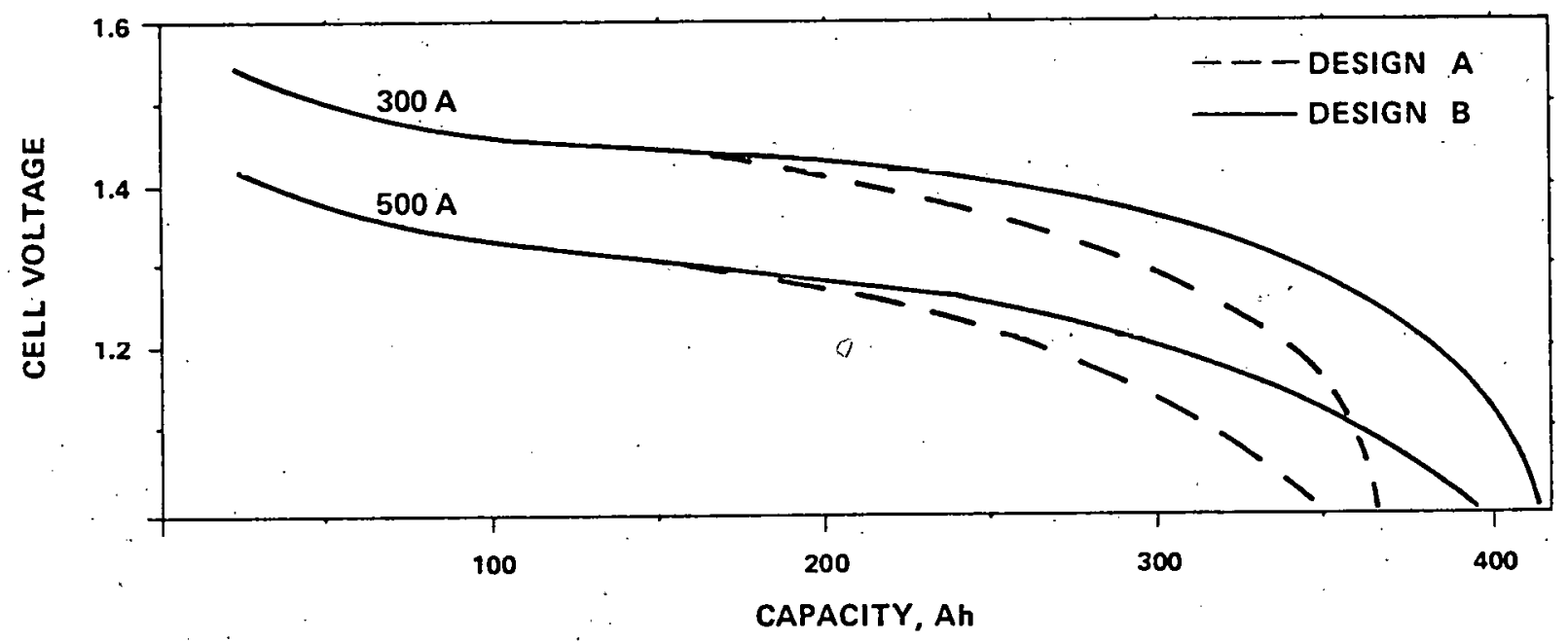

FIGURE 1-3:

TYPICAL DISCHARGE CHARACTERISTICS OF GENERATION I EV Ni-Zn CELL. 


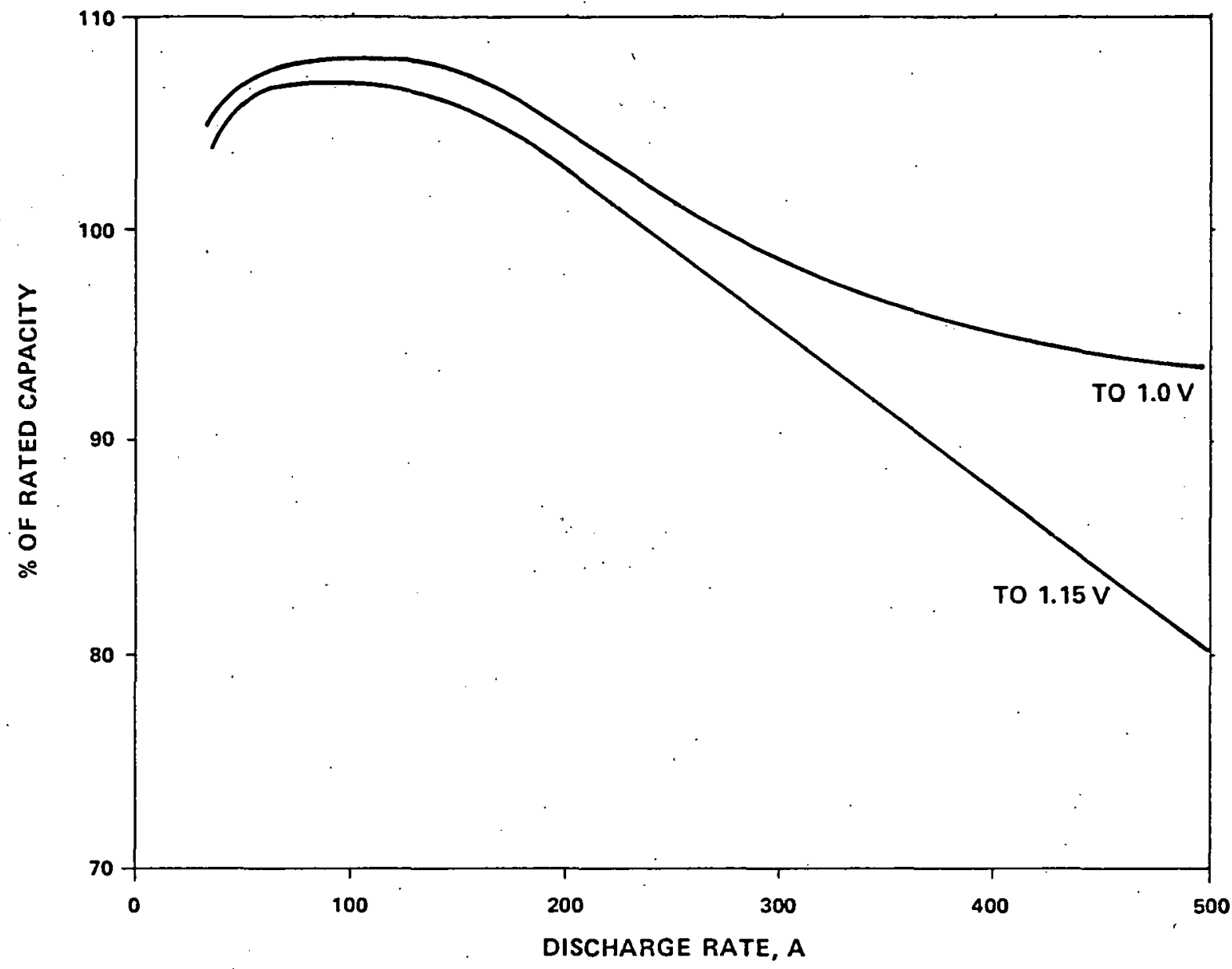

FIGURE 1.4:

GENERATION I ELECTRIC VEHICLE Ni-Zn CELL - CAPACITY AS A FUNCTION OF DISCHARGE RATE.

Design A / Rated Capacity - 370 Ah at 3 to 6 Hour Rates. 


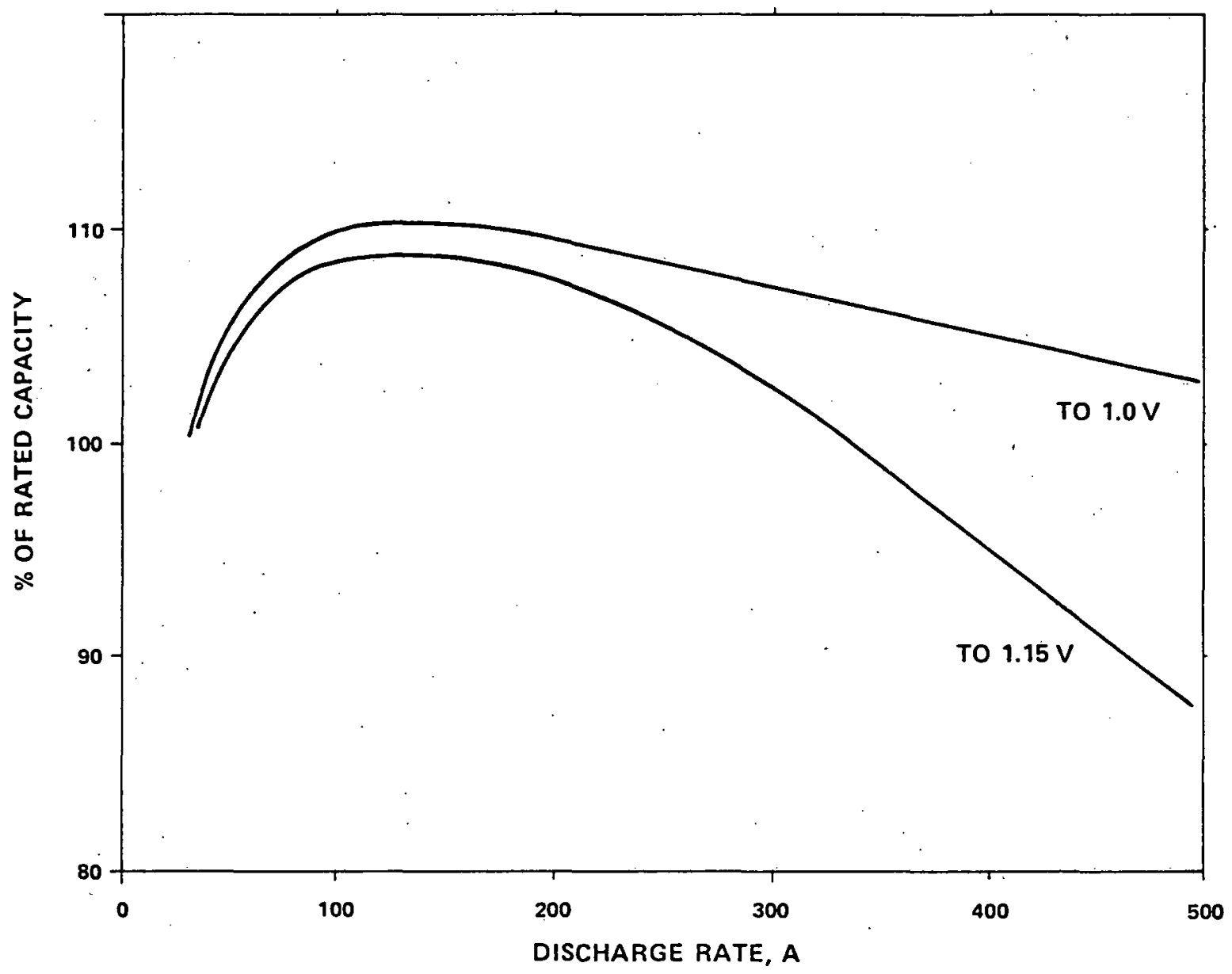

FIGURE 1-5:

GENERATION I ELECTRIC VEHICLE Ni-Zn CELL - CAPACITY AS A FUNCTION OF DISCHARGE RATE.

Design B / Rated Capacity - 385 Ah at 3 to 6 Hour Rates. 


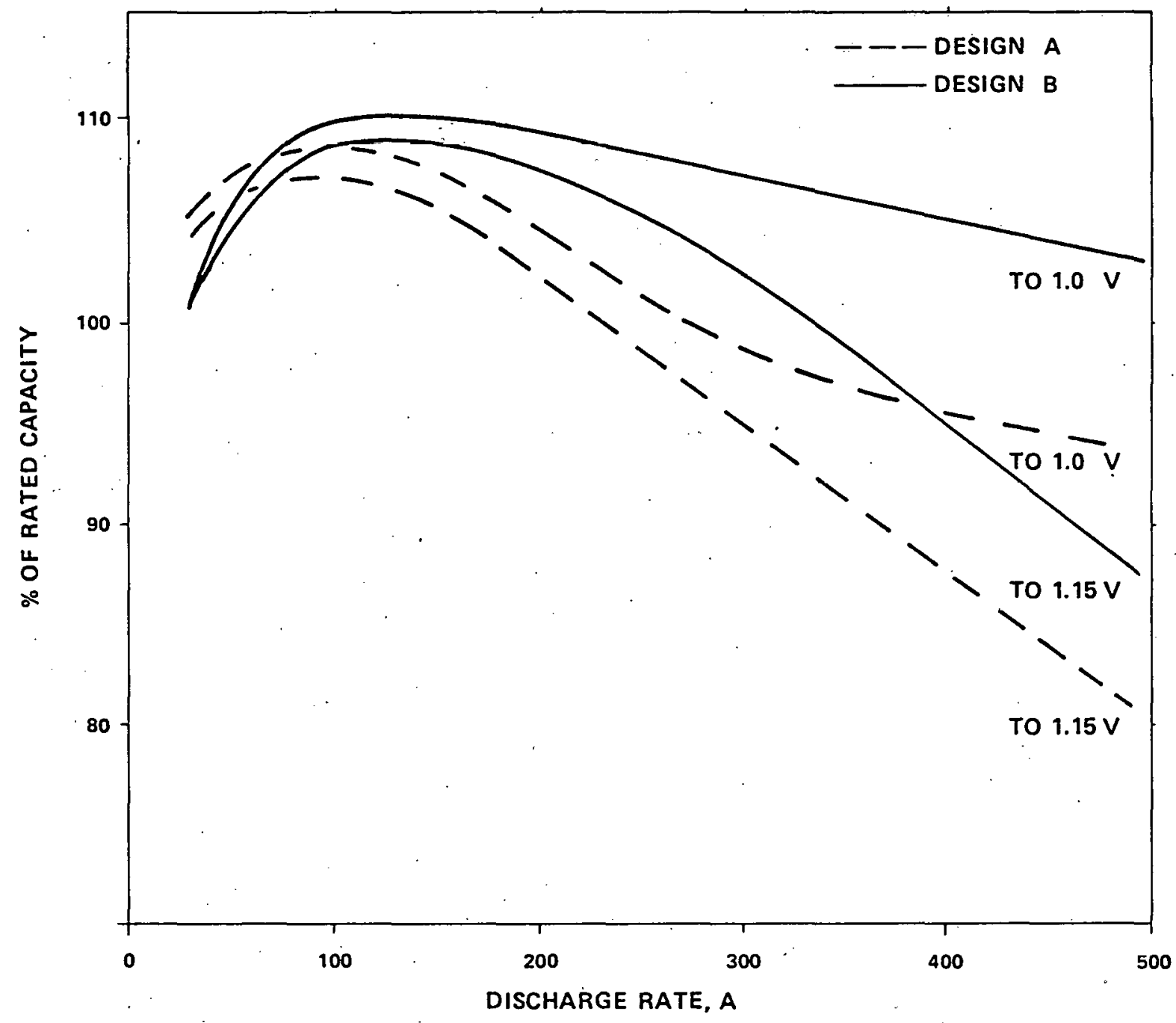

FIGURE 1.6:

GENERATION I ELECTRIC VEHICLE Ni-Zn CELL.

Comparison of Designs $A$ and $B$. 


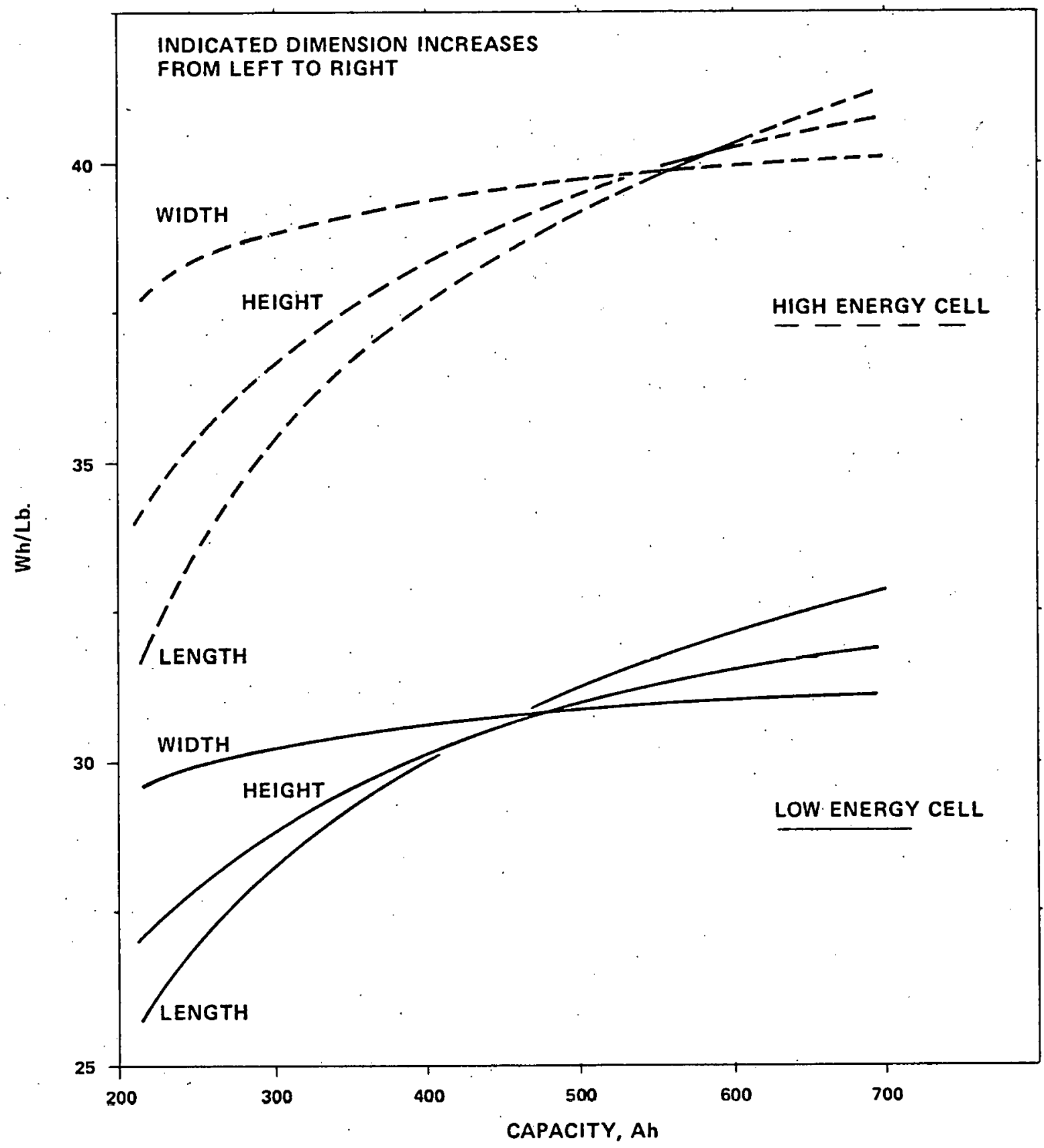

FIGURE 1-7:

SPECIFIC ENERGY AS A FUNCTION OF CAPACITY. 6 Hour Rate. 


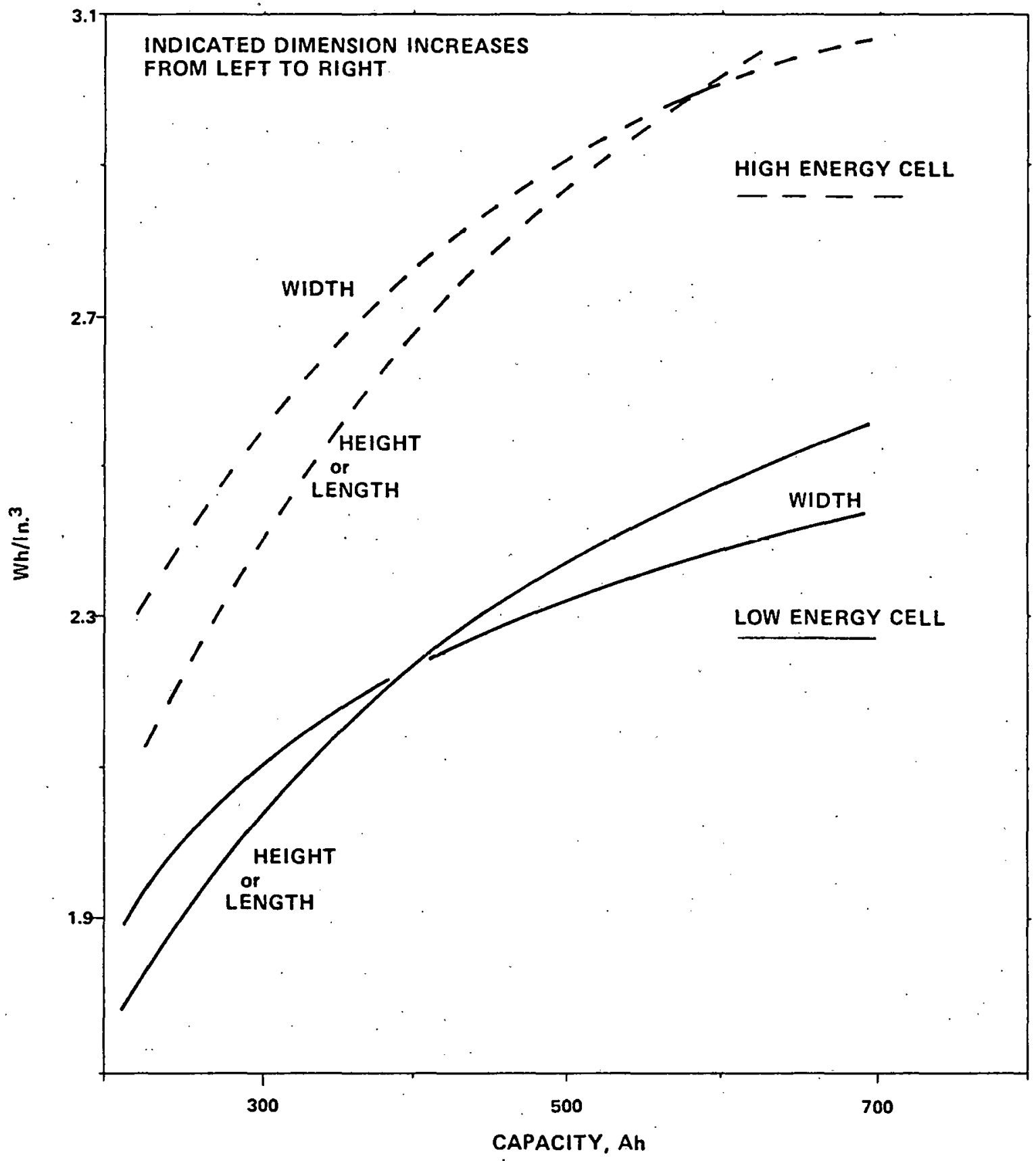

FIGURE 1-8:

VOLUMETRIC ENERGY AS A FUNCTION OF CAPACITY.

6. Hour Rate. 


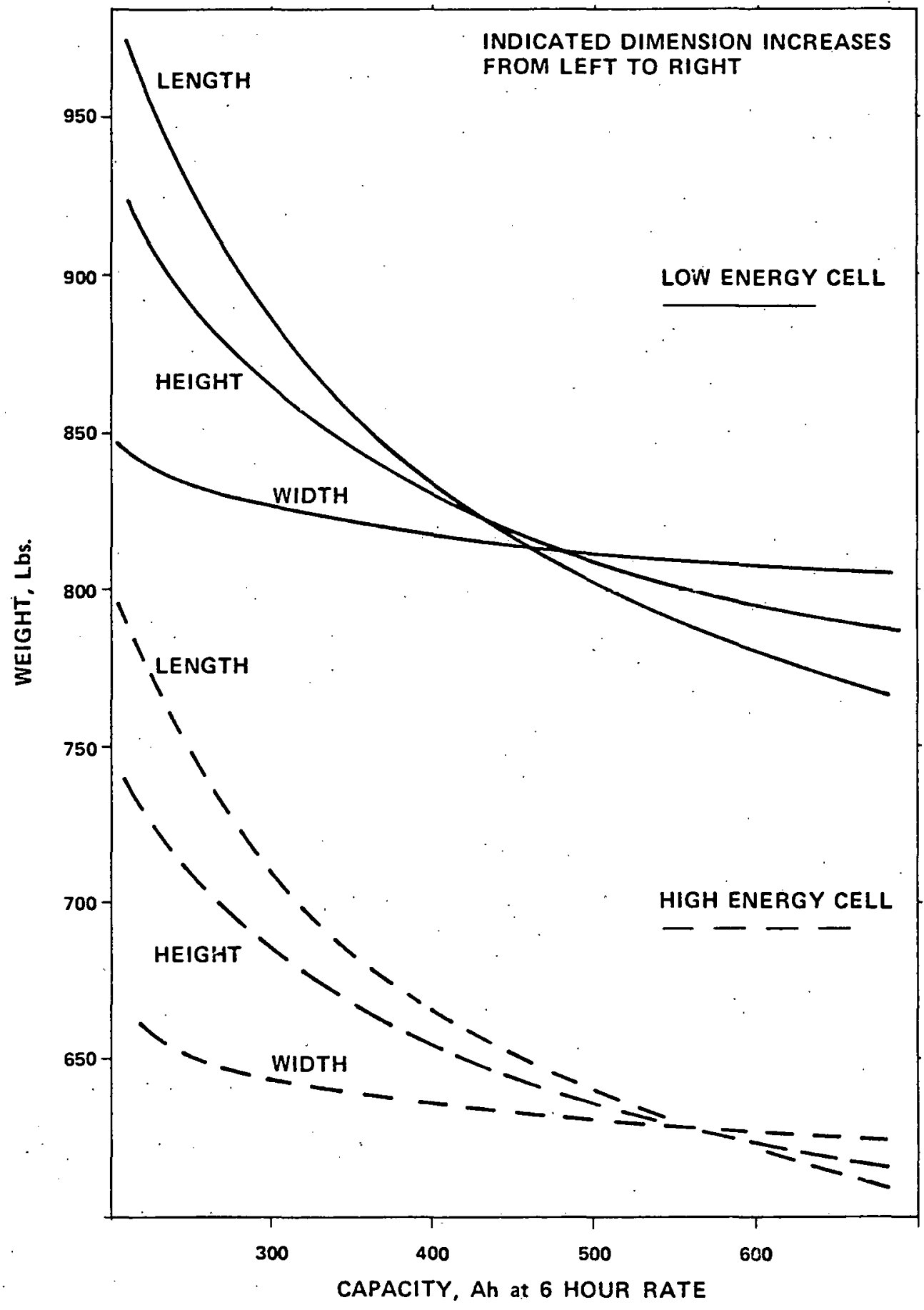

FIGURE 1-9:

BATTERY WEIGHT AS A FUNCTION OF CAPACITY.

25 Kwh Batteries / Excludes Battery Packaging. 


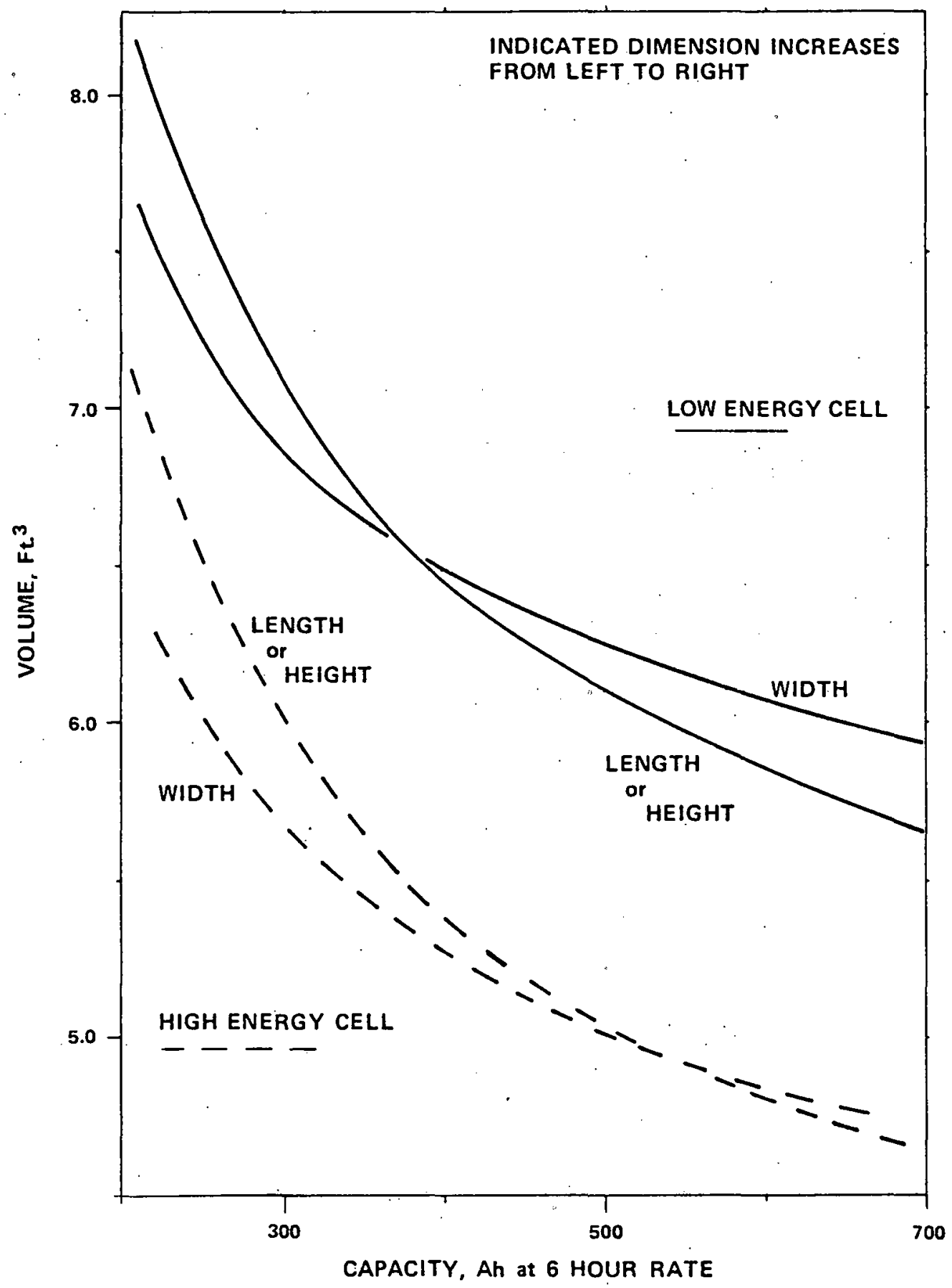

FIGURE 1.10:

BATTERY VOLUME AS A FUNCTION OF CAPACITY.

$25 \mathrm{kWh}$ Batteries / Excludes Battery Packaging. 


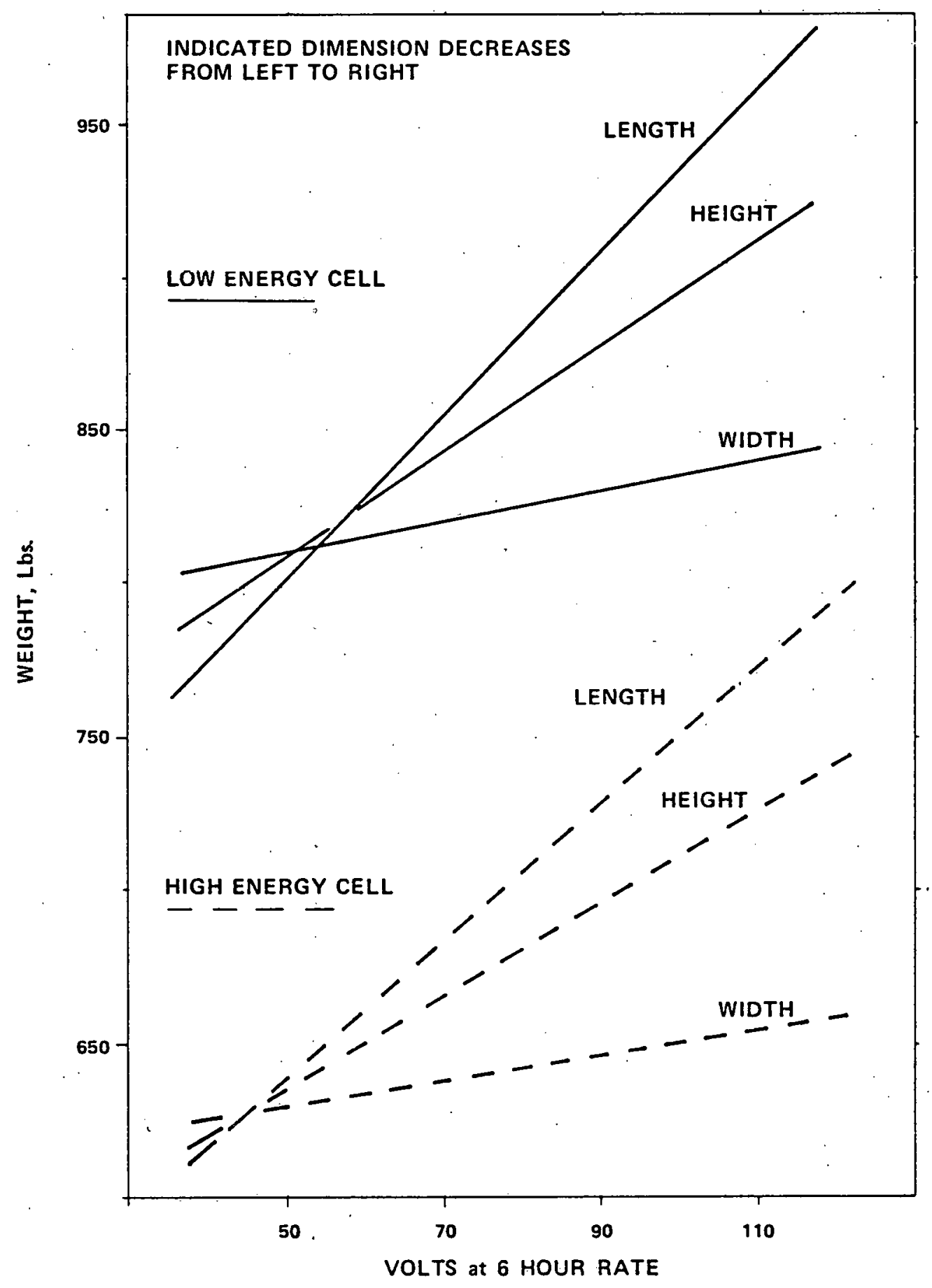

FIGURE 1.11.

BATTERY WEIGHT AS A FUNCTION OF VOLTAGE.

$25 \mathrm{kWh}$ Batteries / Excludes Battery Packaging. 


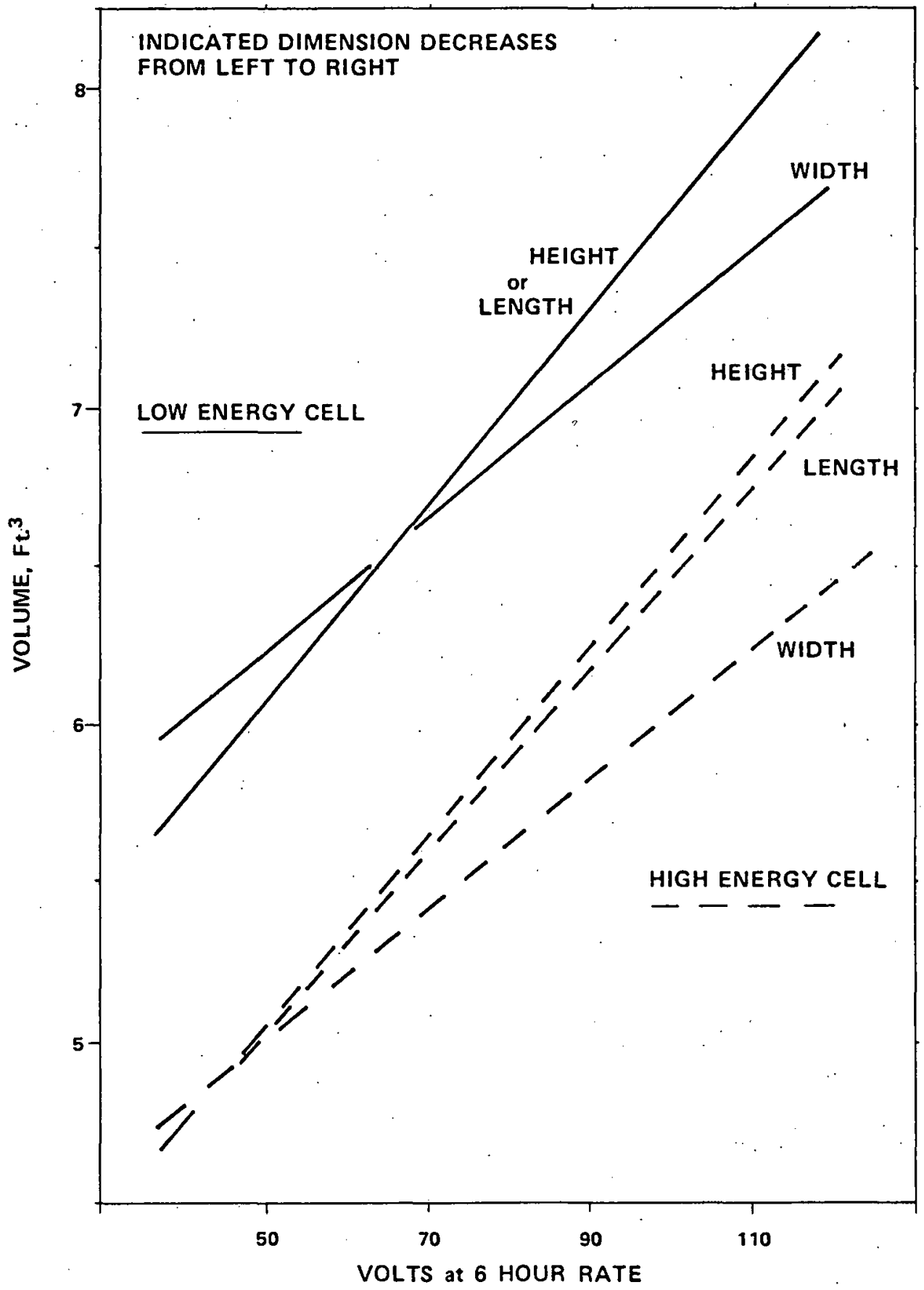

FIGURE 1-12:

BATTERY VOLUME AS A FUNCTION OF VOLTAGE.

25 kWh Batteries / Excludes Battery Packaging. 


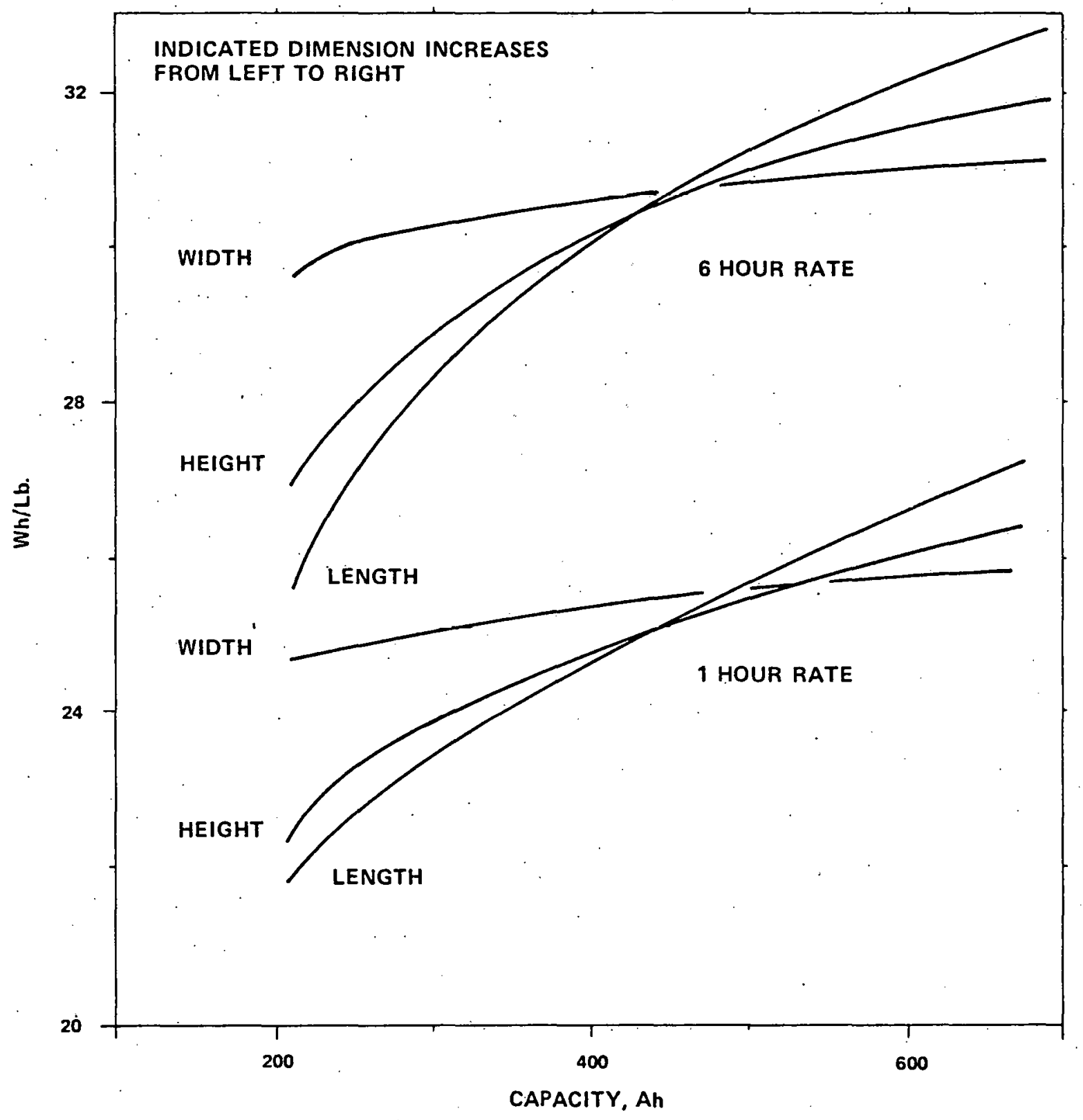

FIGURE 1.13:

SPECIFIC ENERGY AS A FUNCTION OF CAPACITY. Low Energy Cells. 


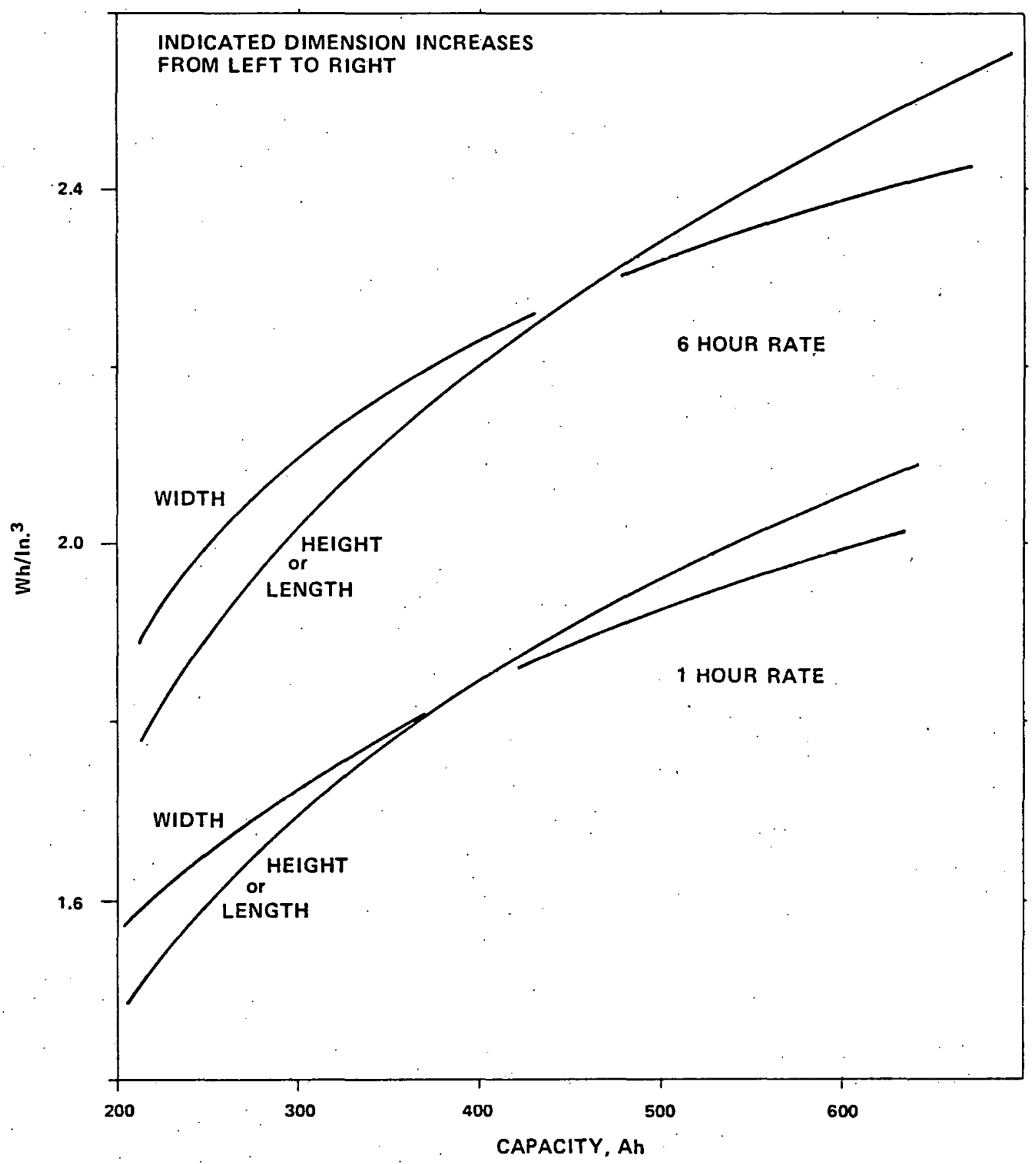

FIGURE 1-14:

VOLUMETRIC ENERGY AS A FUNCTION OF CAPACITY. Low Energy Cells. 


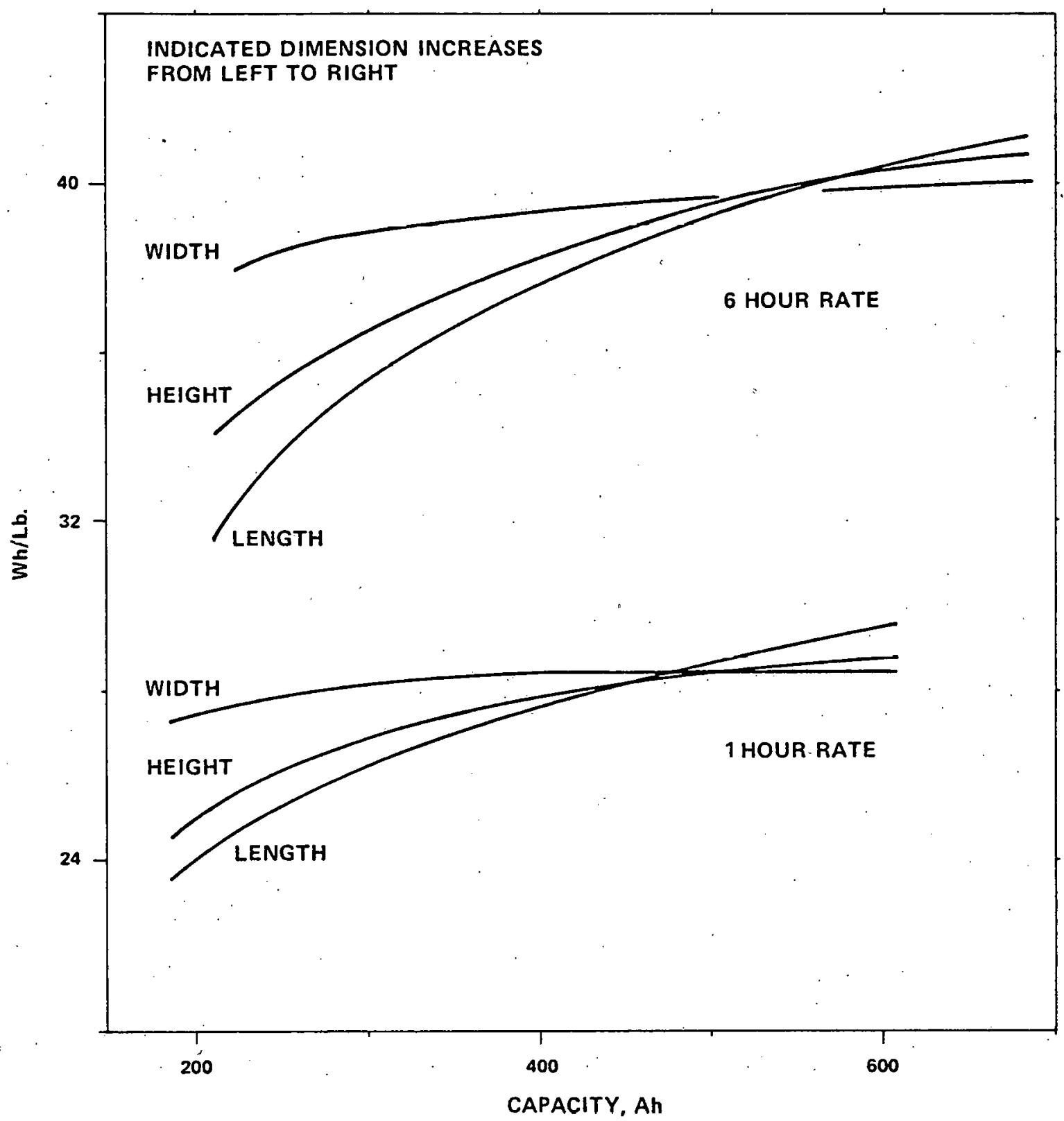

FIGURE 1.15:

SPECIFIC ENERGY AS A FUNCTION OF CAPACITY.

High Energy Cells. 


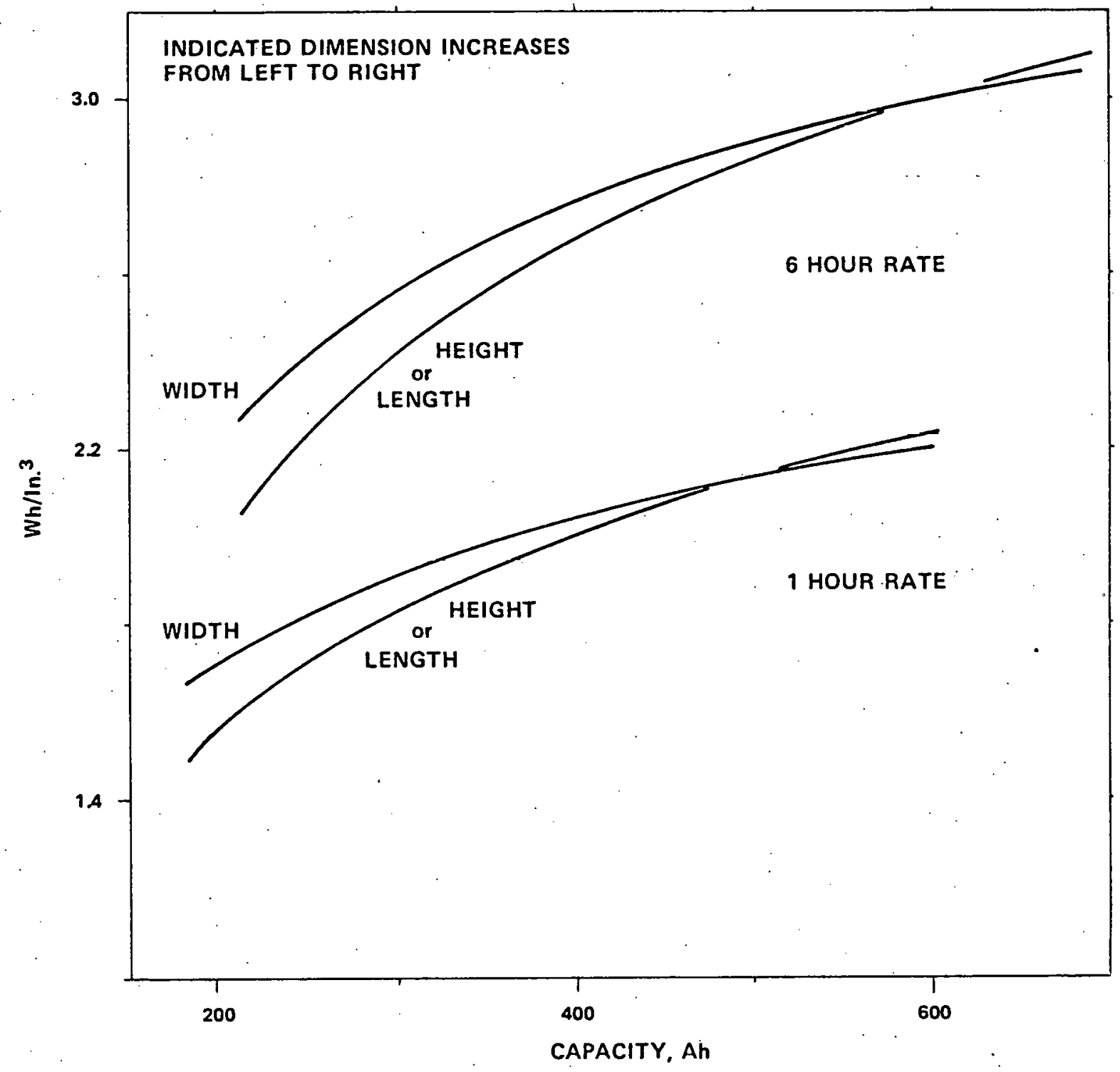

FIGURE 1-16:

VOLUMETRIC ENERGY AS A FUNCTION OF CAPACITY.

High Energy Cells. 


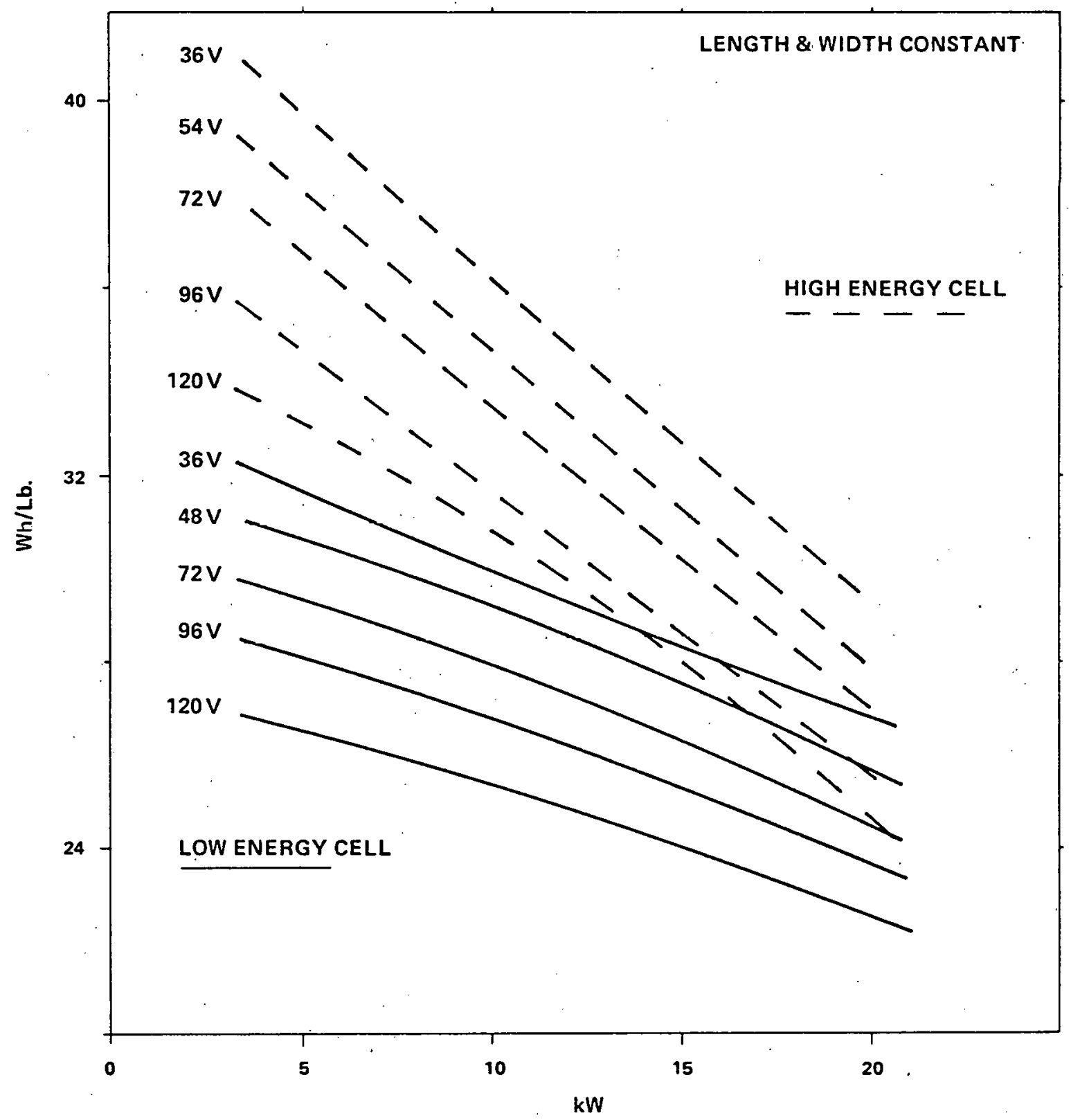

FIGURE 1.17:

SPECIFIC ENERGY AS A FUNCTION OF DISCHARGE RATE. 


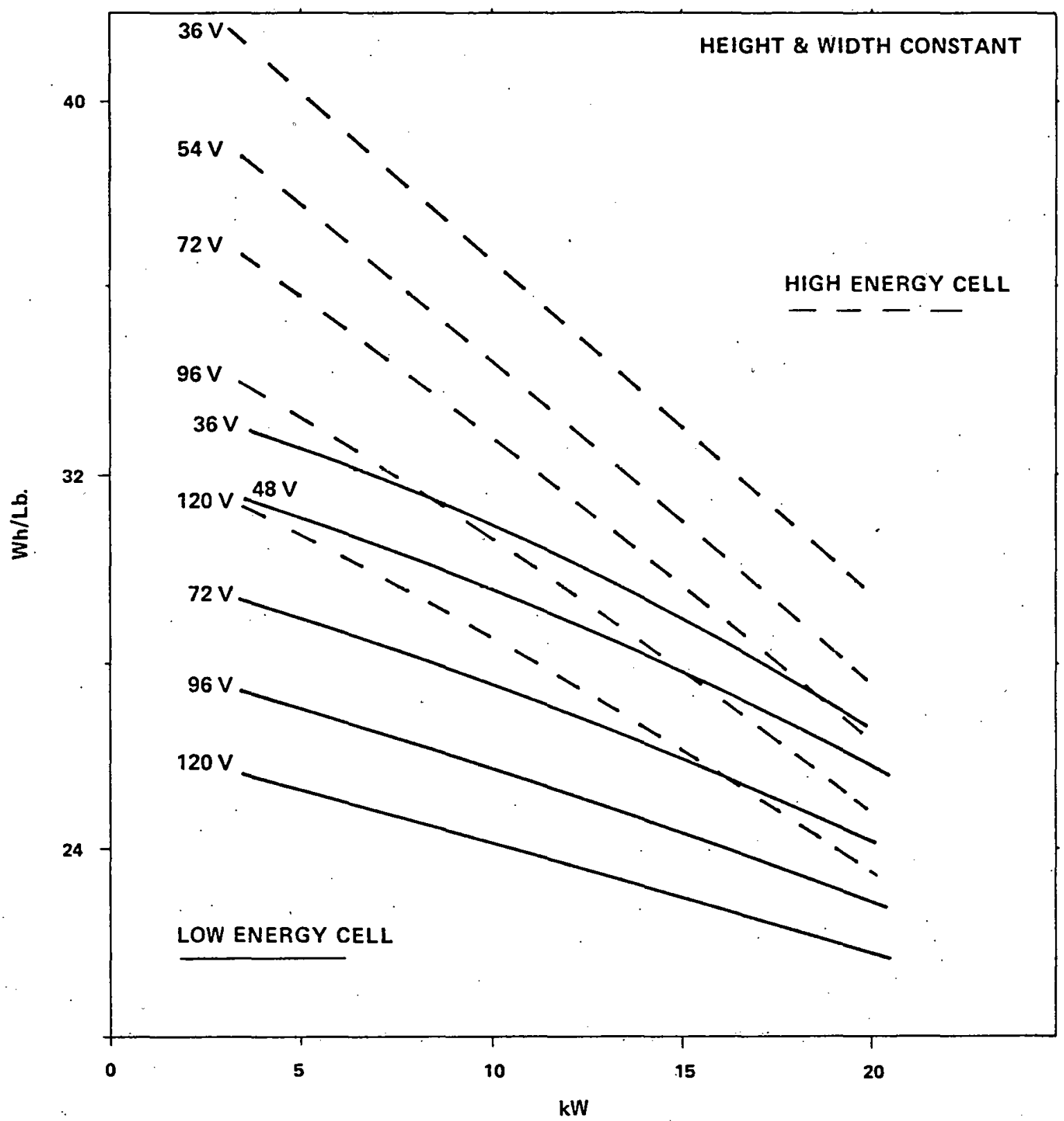

FIGURE 1.18:

SPECIFIC ENERGY AS A FUNCTION OF DISCHARGE RATE. 


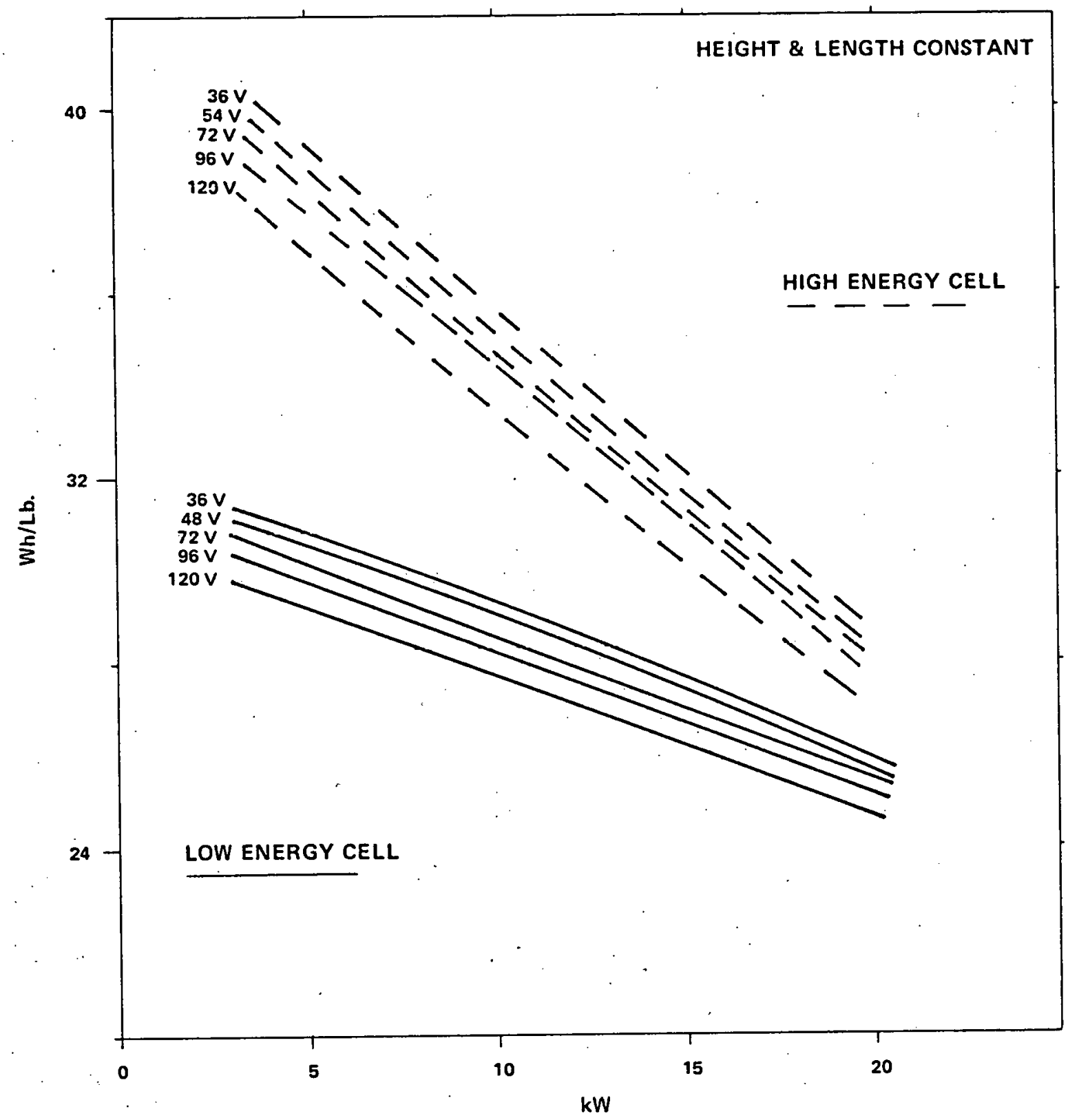

FIGURE 1-19:

SPECIFIC ENERGY AS A FUNCTION OF DISCHARGE RATE. 


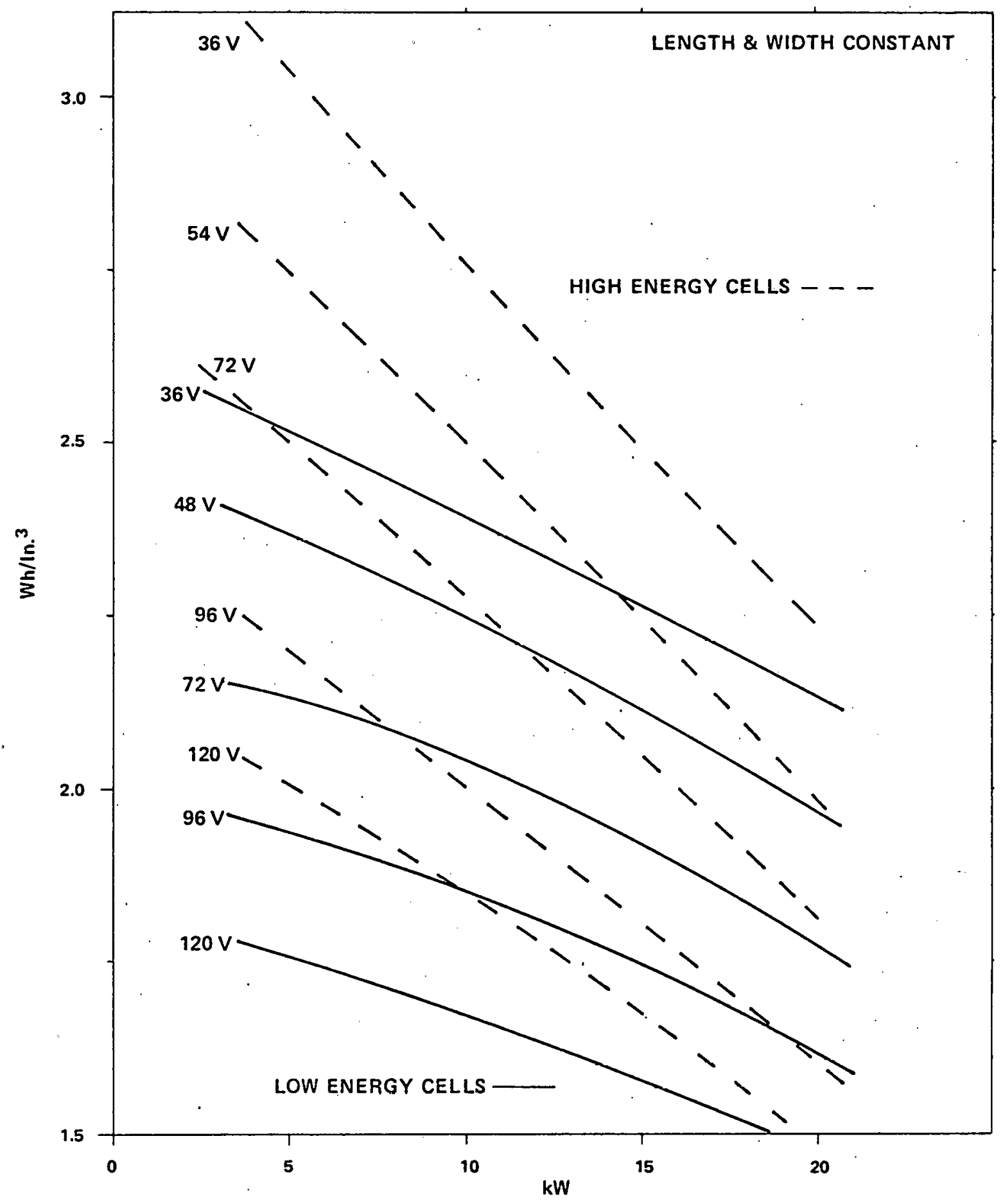

FIGURE 1.20:

VOLUMETRIC ENERGY AS A FUNCTION OF DISCHARGE RATE. 


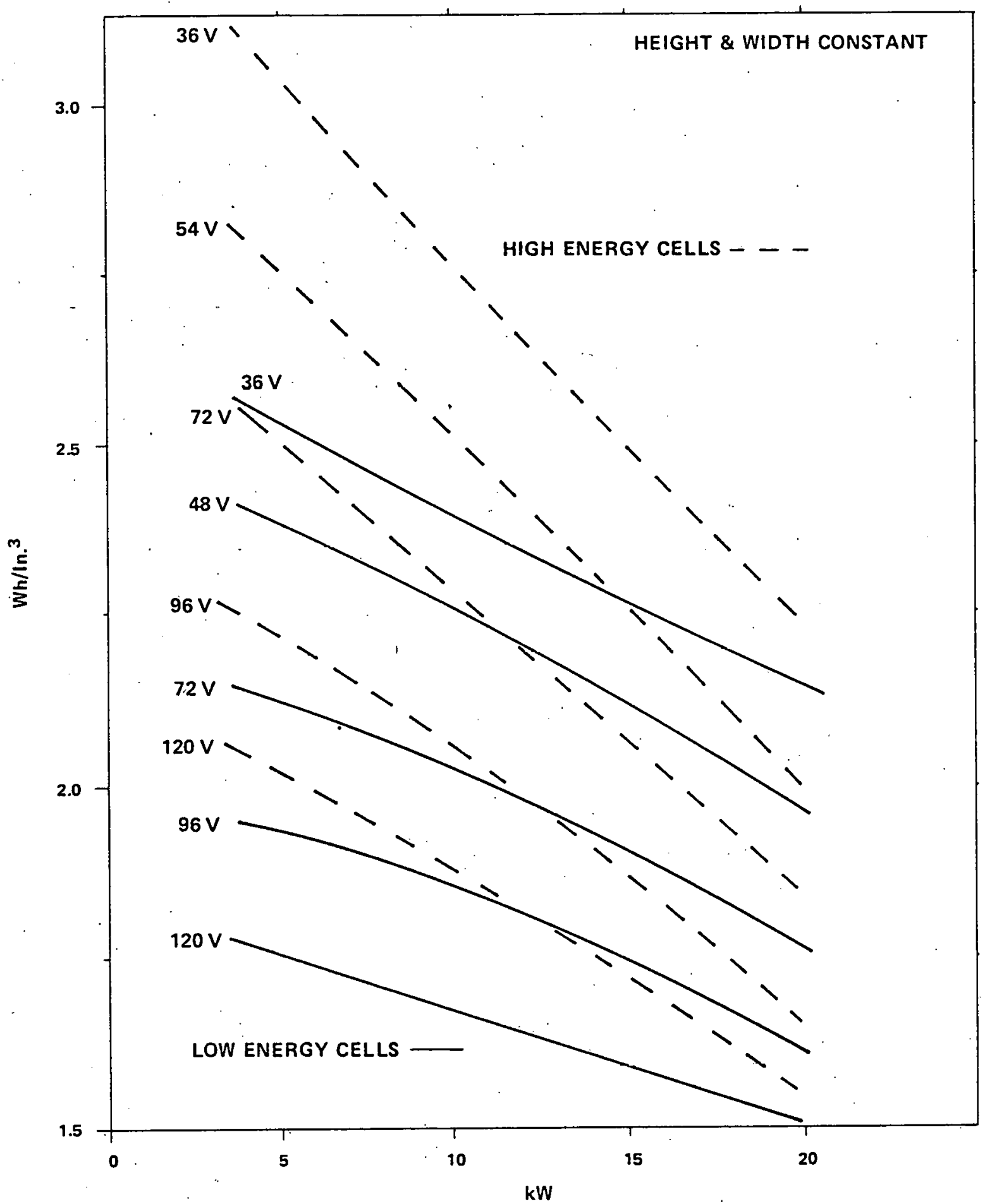

FIGURE 1.21:

VOLUMETRIC ENERGY AS A FUNCTION OF DISCHARGE RATE. 


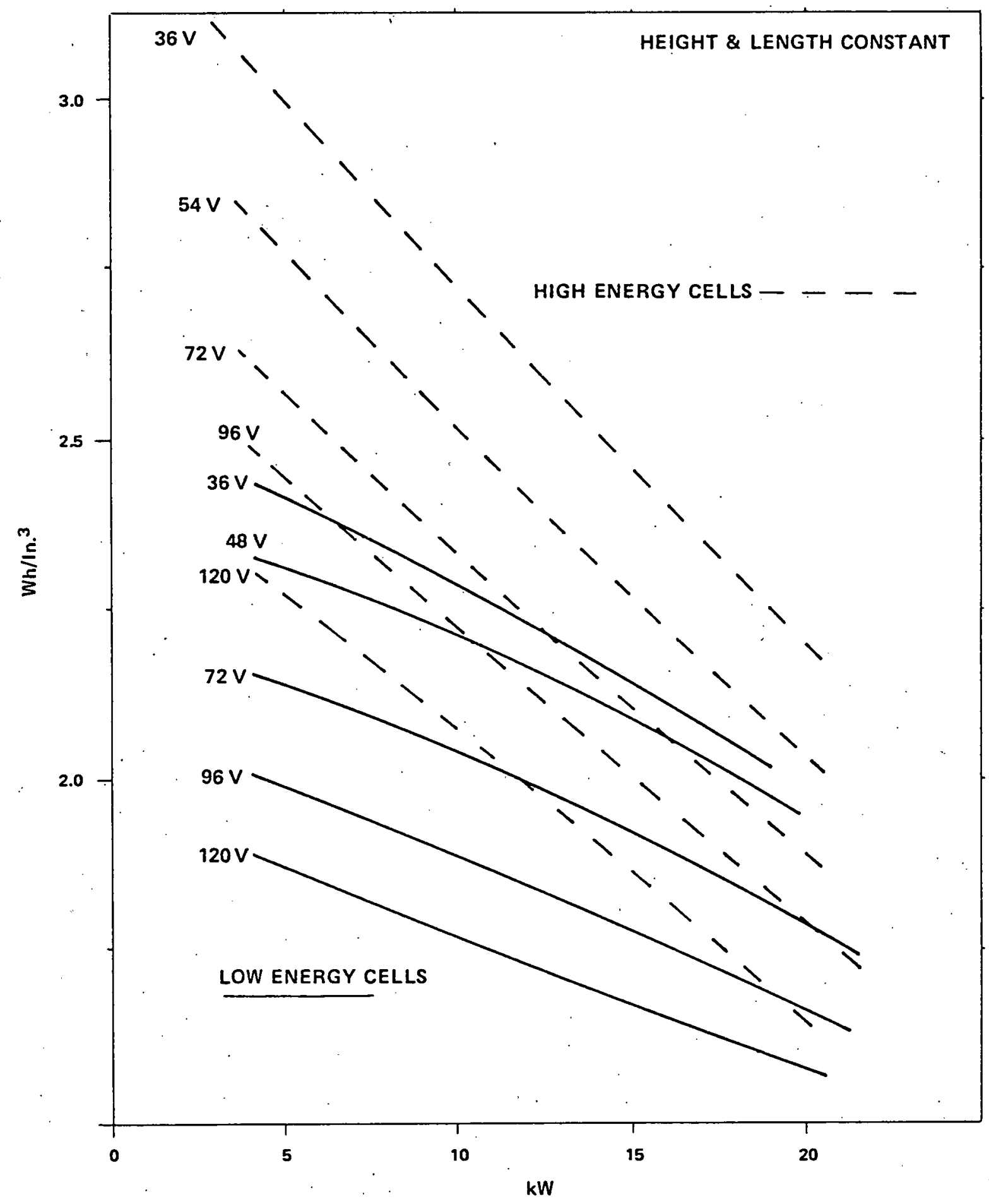

FIGURE 1-22:

VOLUMETRIC ENERGY AS A FUNCTION OF DISCHARGE RATE. 


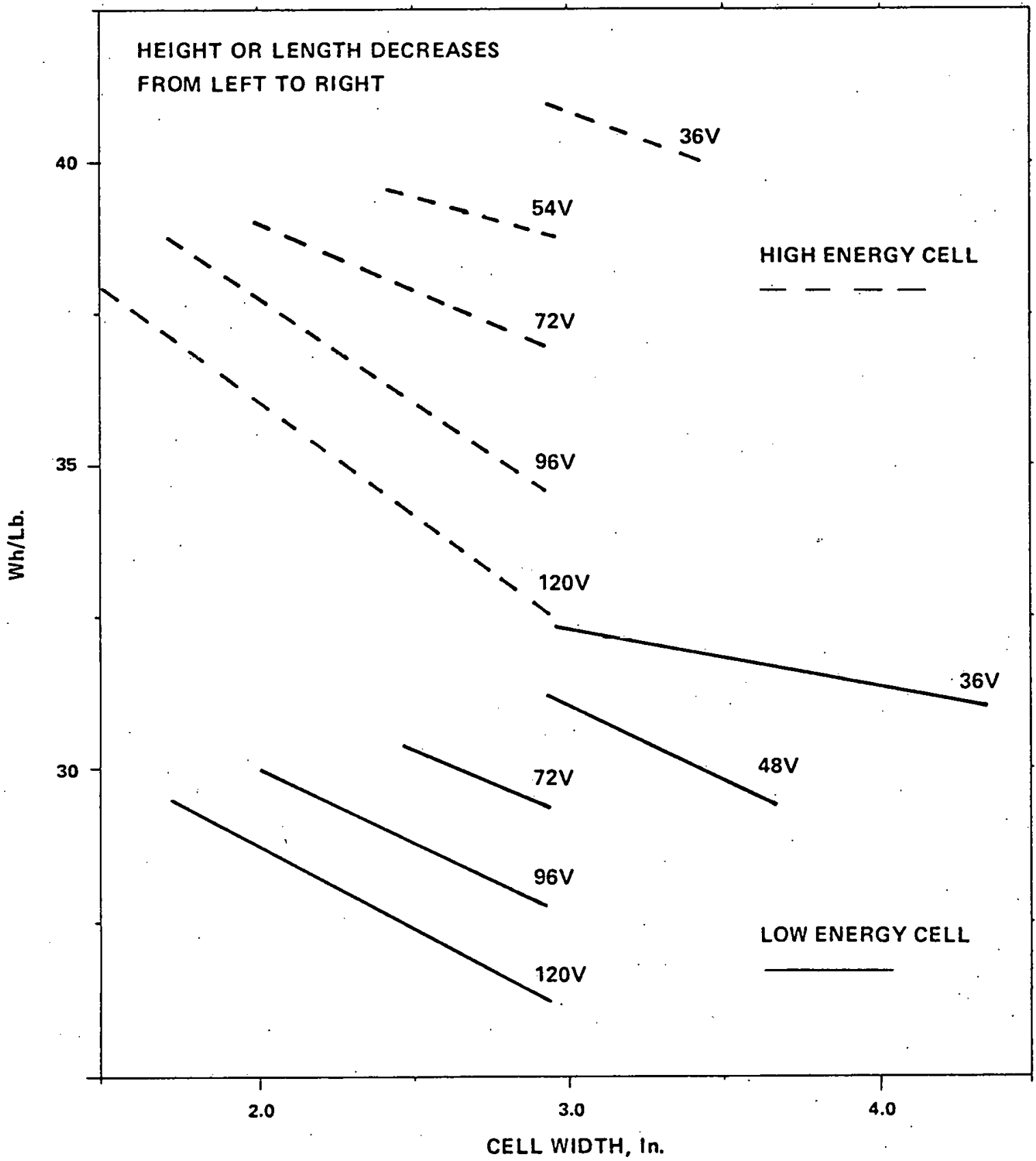

FIGURE 1-23:

SPECIFIC ENERGY AS A FUNCTION OF CELL WIDTH. 


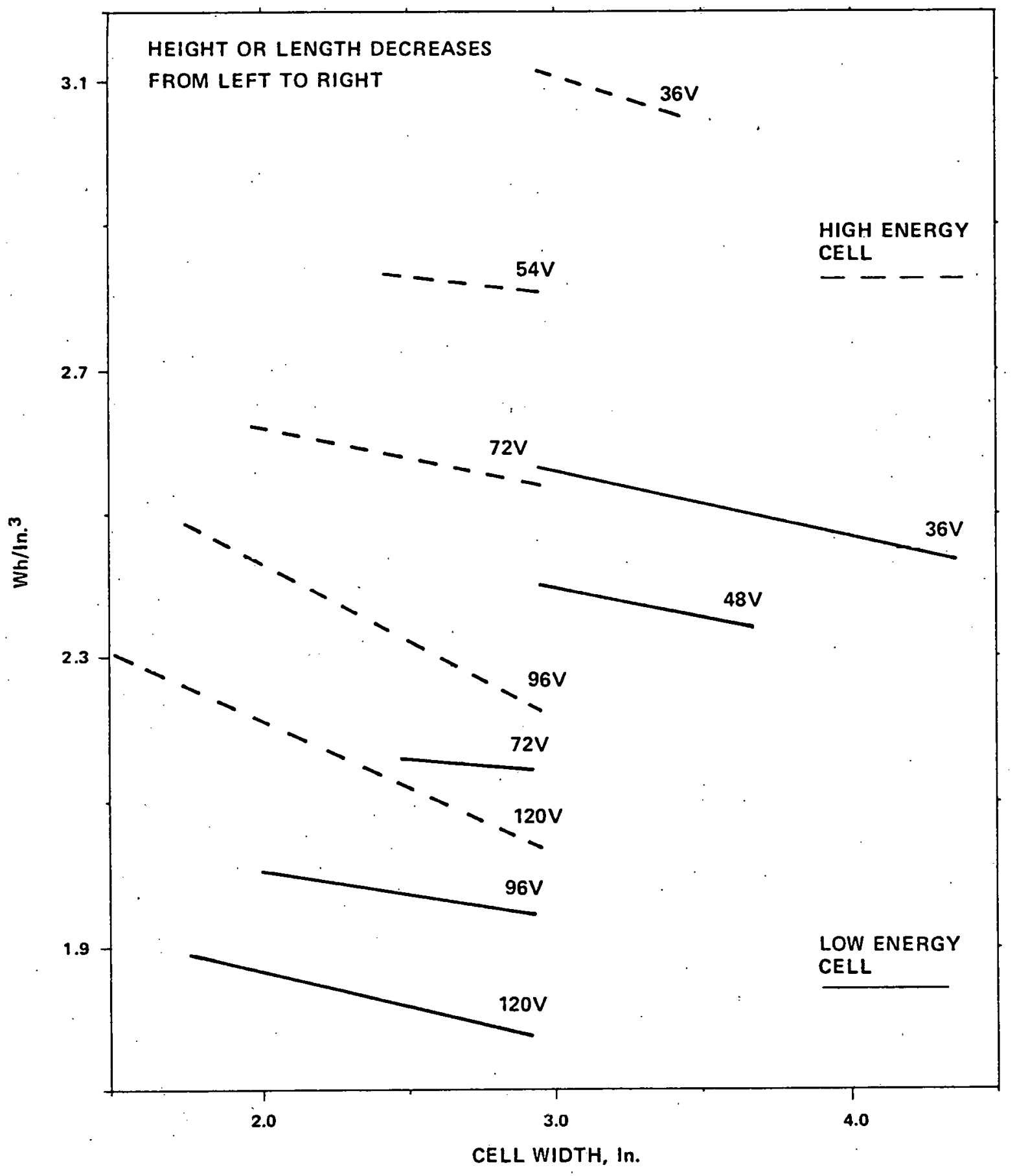

FIGURE 1.24:

VOLUMETRIC ENERGY AS A FUNCTION OF CELL WIDTH. 

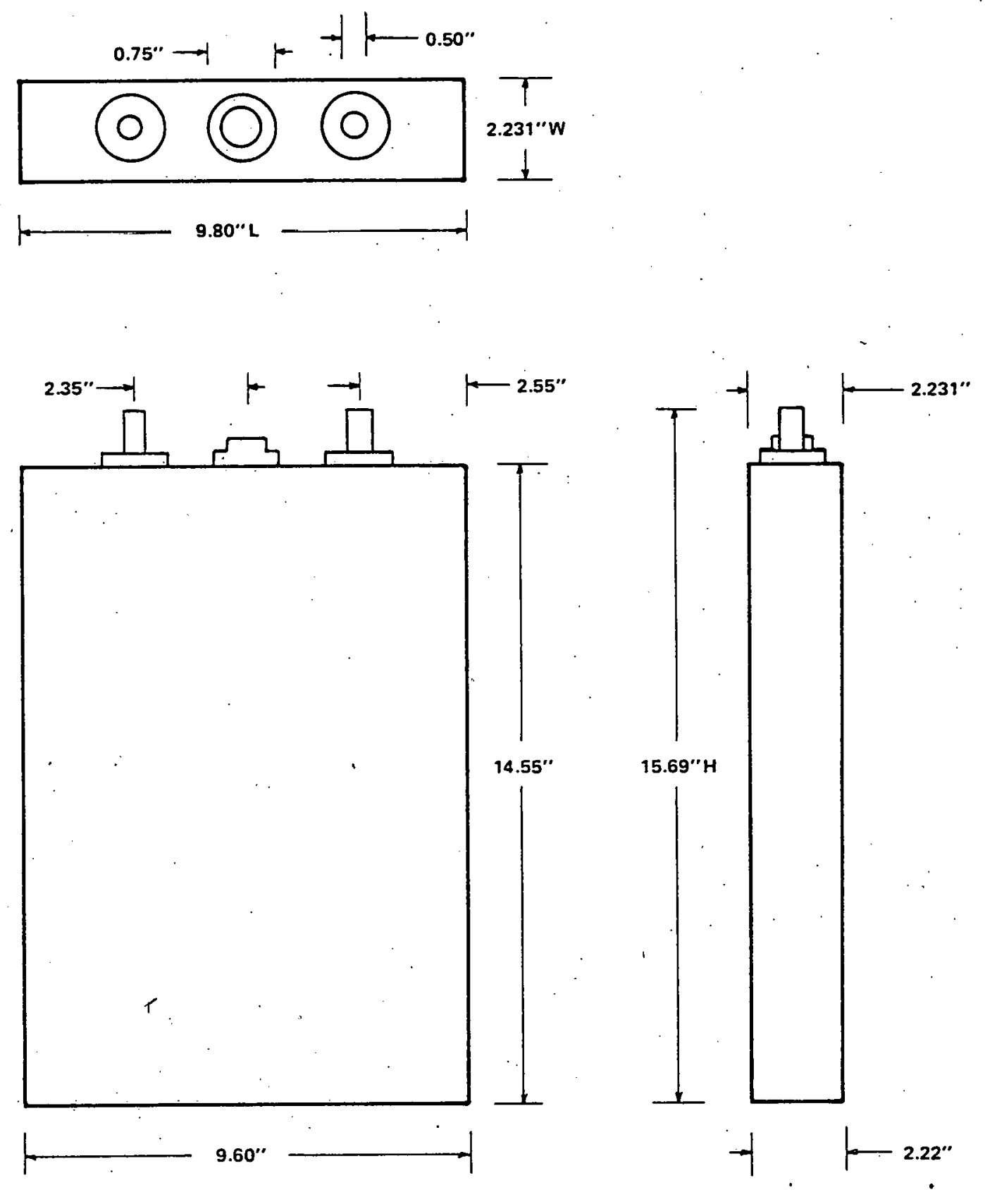

FIGURE 1-25:

DIMENSIONS OF PROPOSED CELL. 


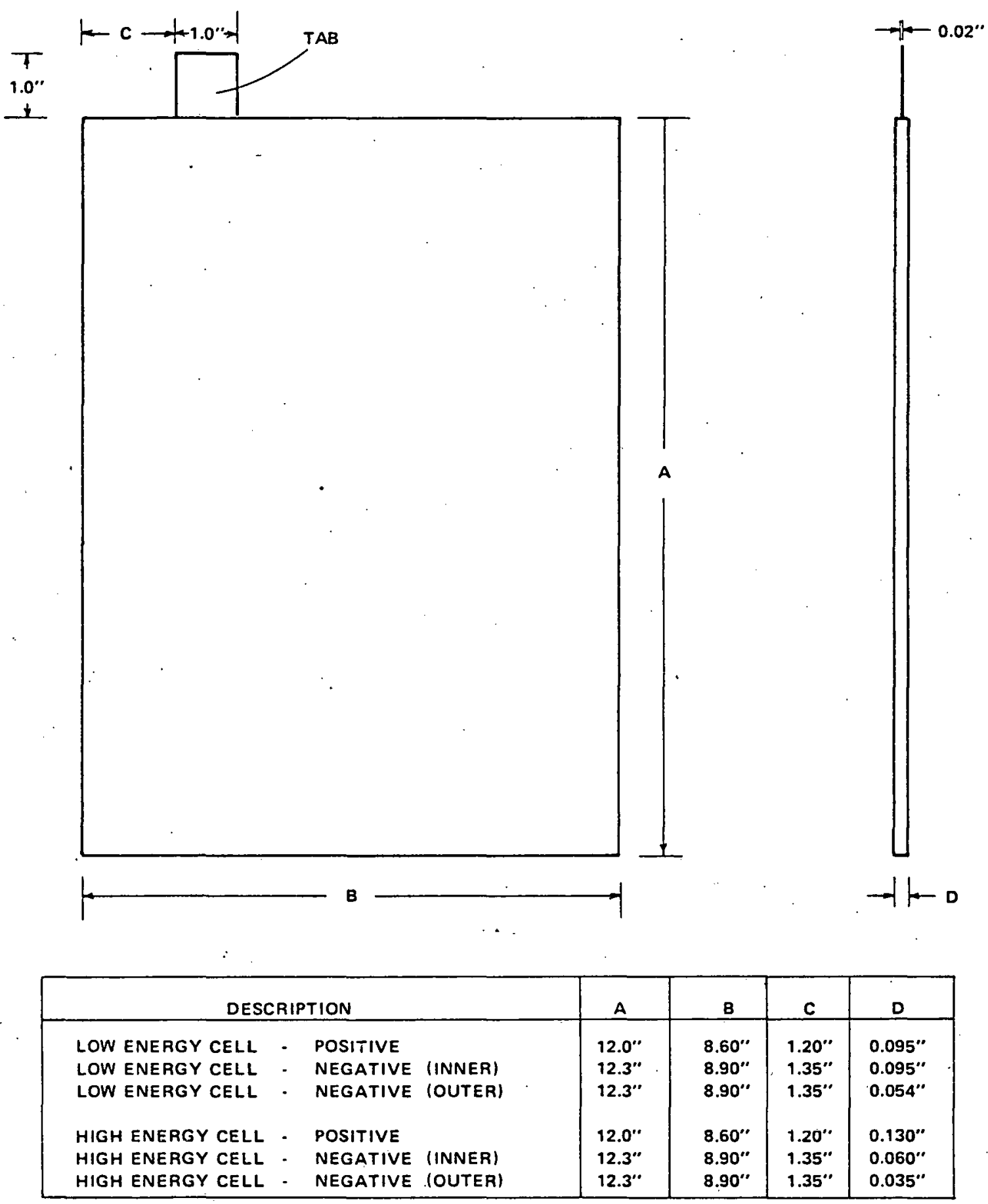

FIGURE 1.26:

ELECTRODE DIMENSIONS FOR PROPOSED CELL. 


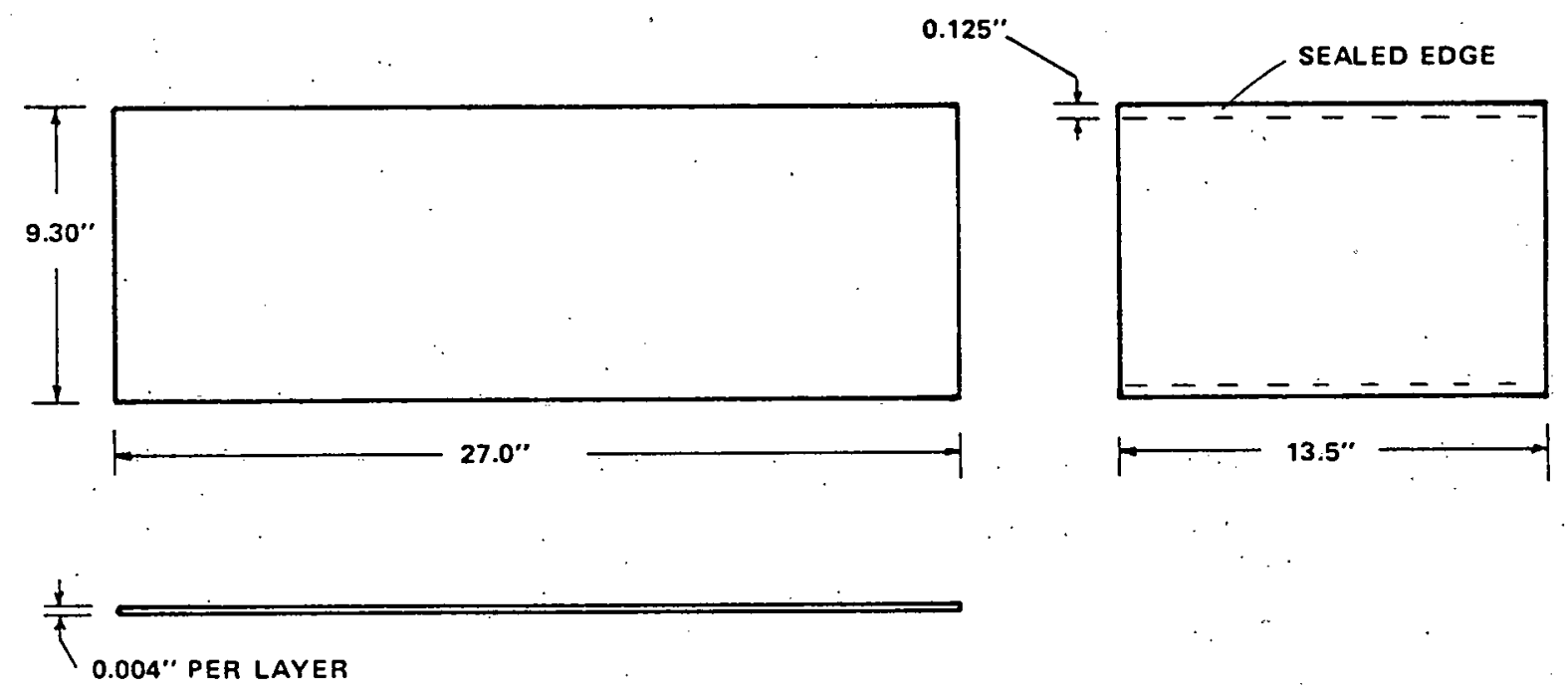

FIGURE 1.27:

SEPARATOR FOR PROPOSED CELL CONFIGURATION. 


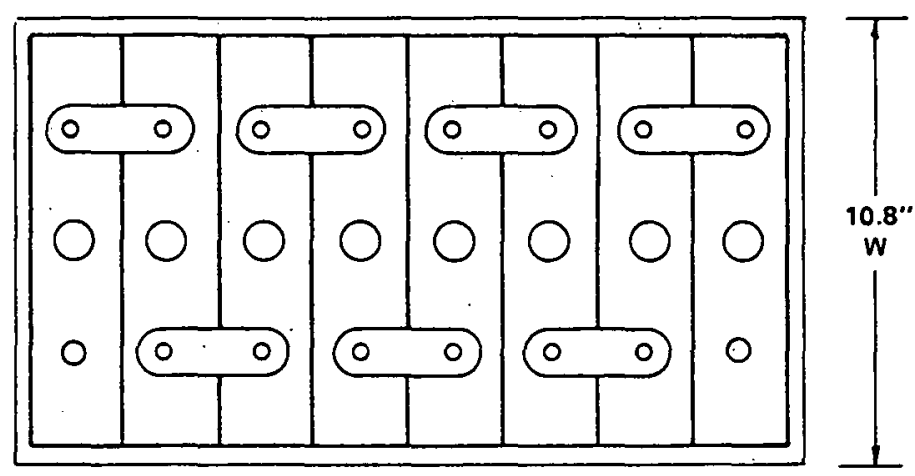

$\rightarrow|2.231 \cdot|$
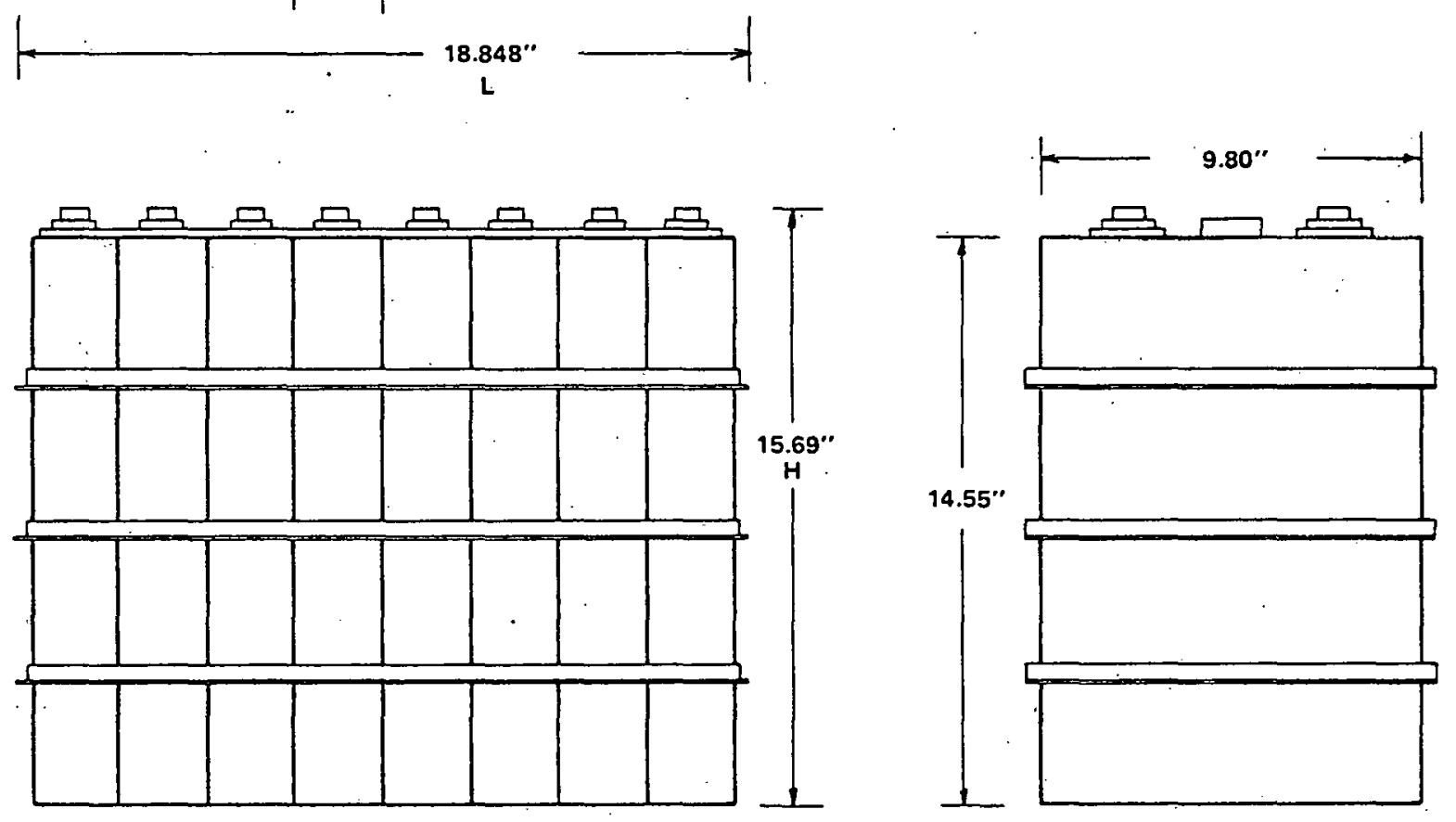

FIGURE 1.28:

DIMENSIONS OF PROPOSED 8 - CELL BATTERY MODULE. 

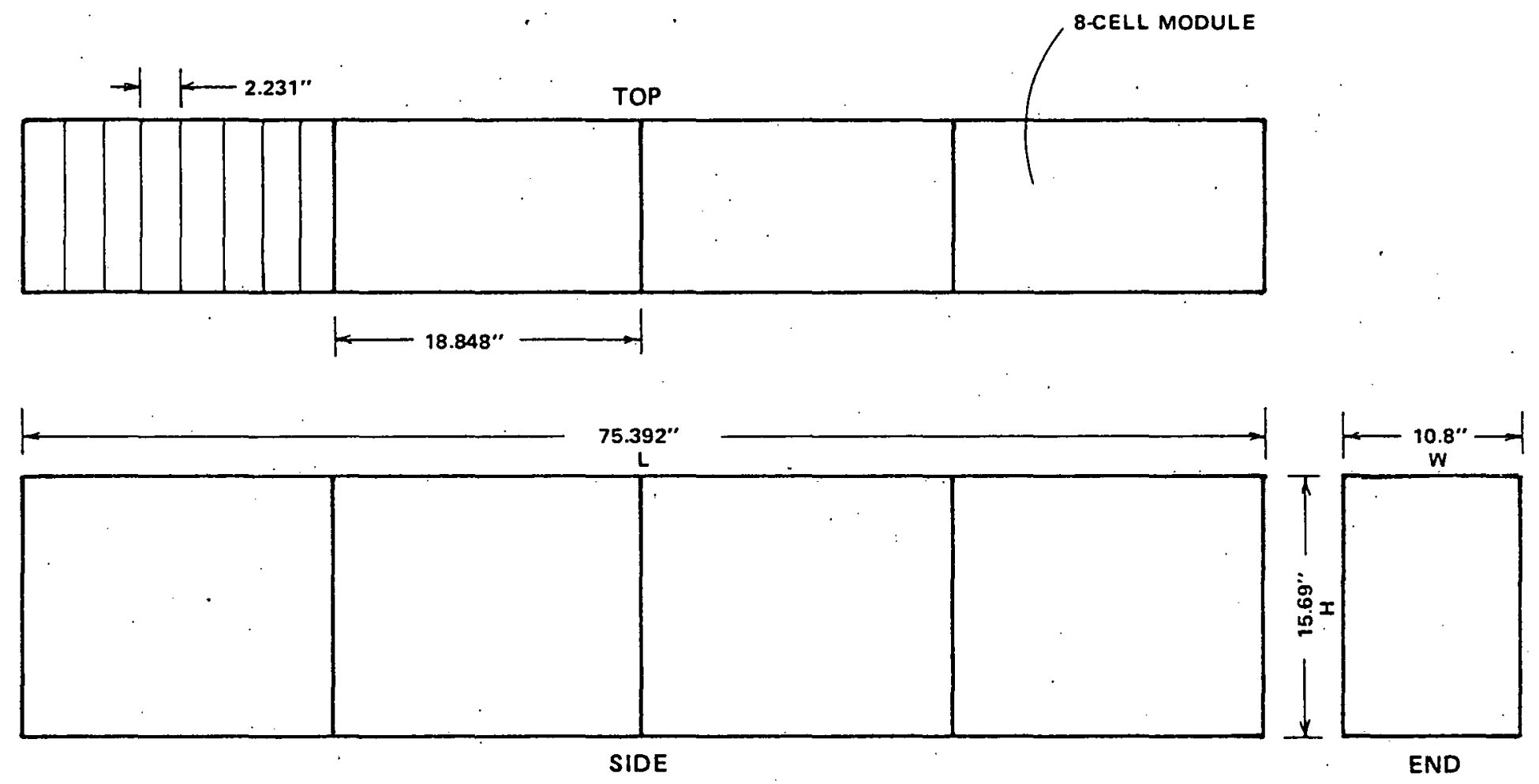

FIGURE 1-29:

DIMENSIONS OF PROPOSED 52 - VOLT BATTERY.

Configuration $\mathbf{A}$. 

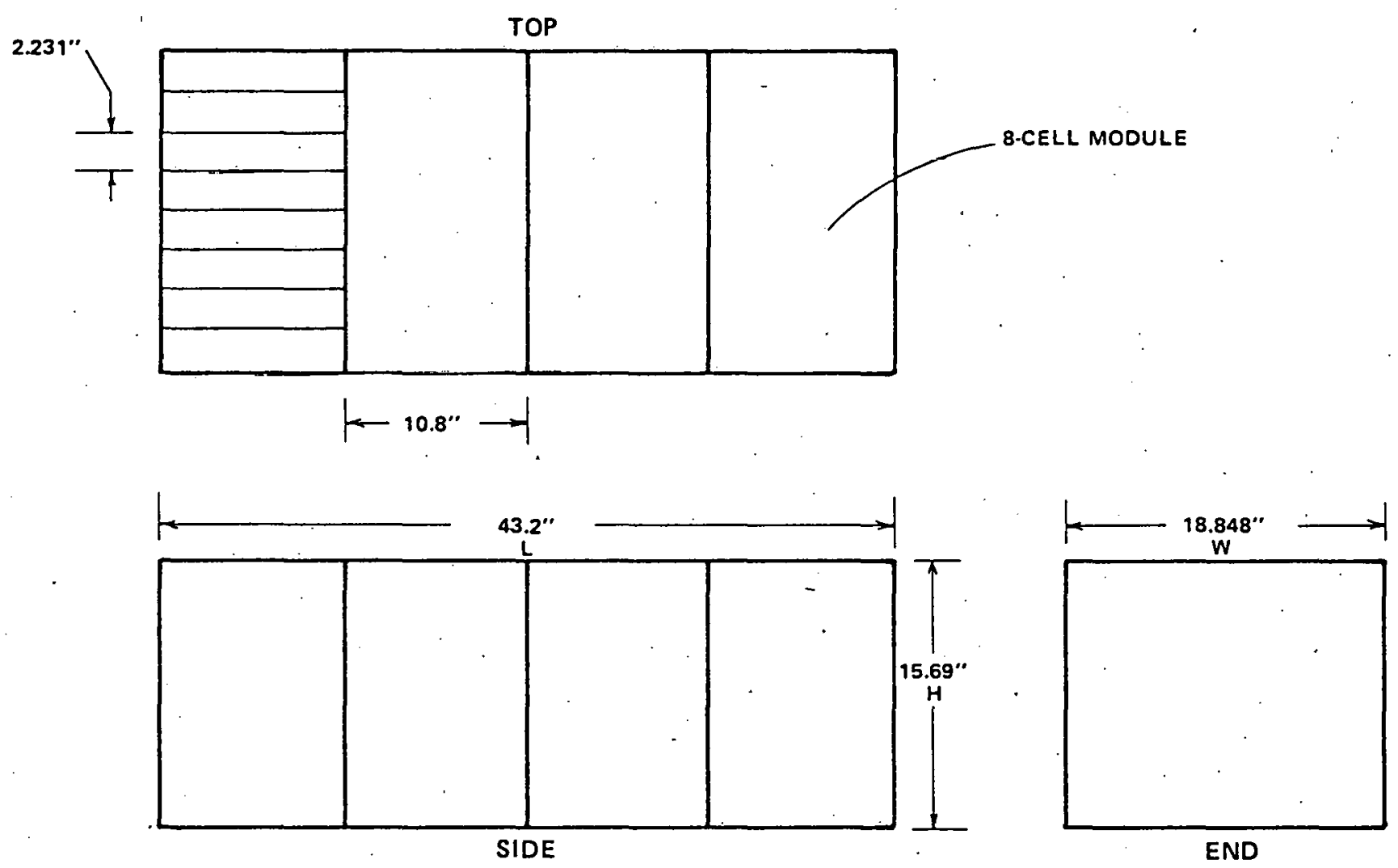

FIGURE 1-30:

DIMENSIONS OF PROPOSED 52 - VOLT BATTERY.

Configuration $\mathrm{B}$. 

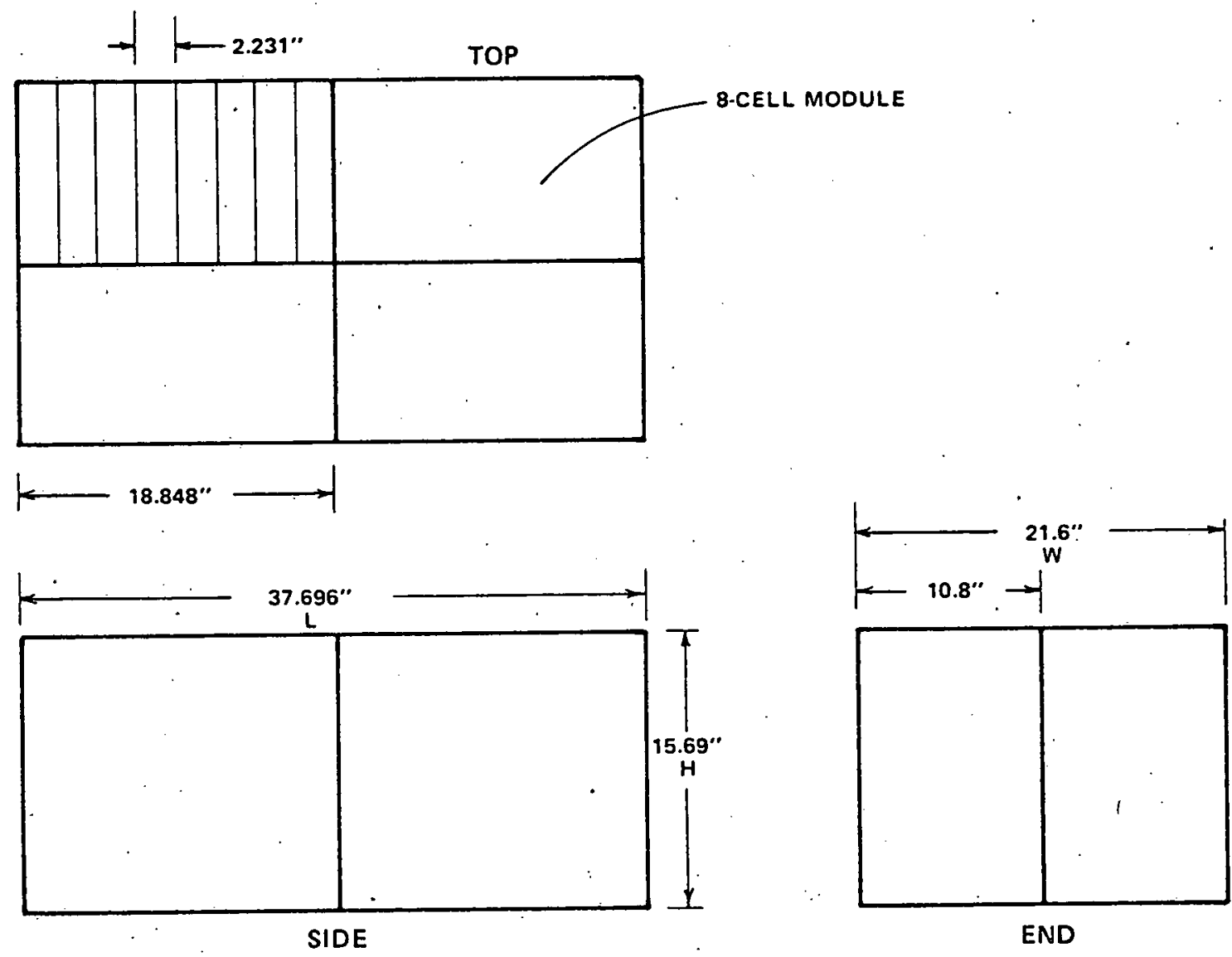

FIGURE 1-31:

DIMENSIONS OF PROPOSED 52 - VOLT BATTERY.

Configuration $\mathrm{C}$. 


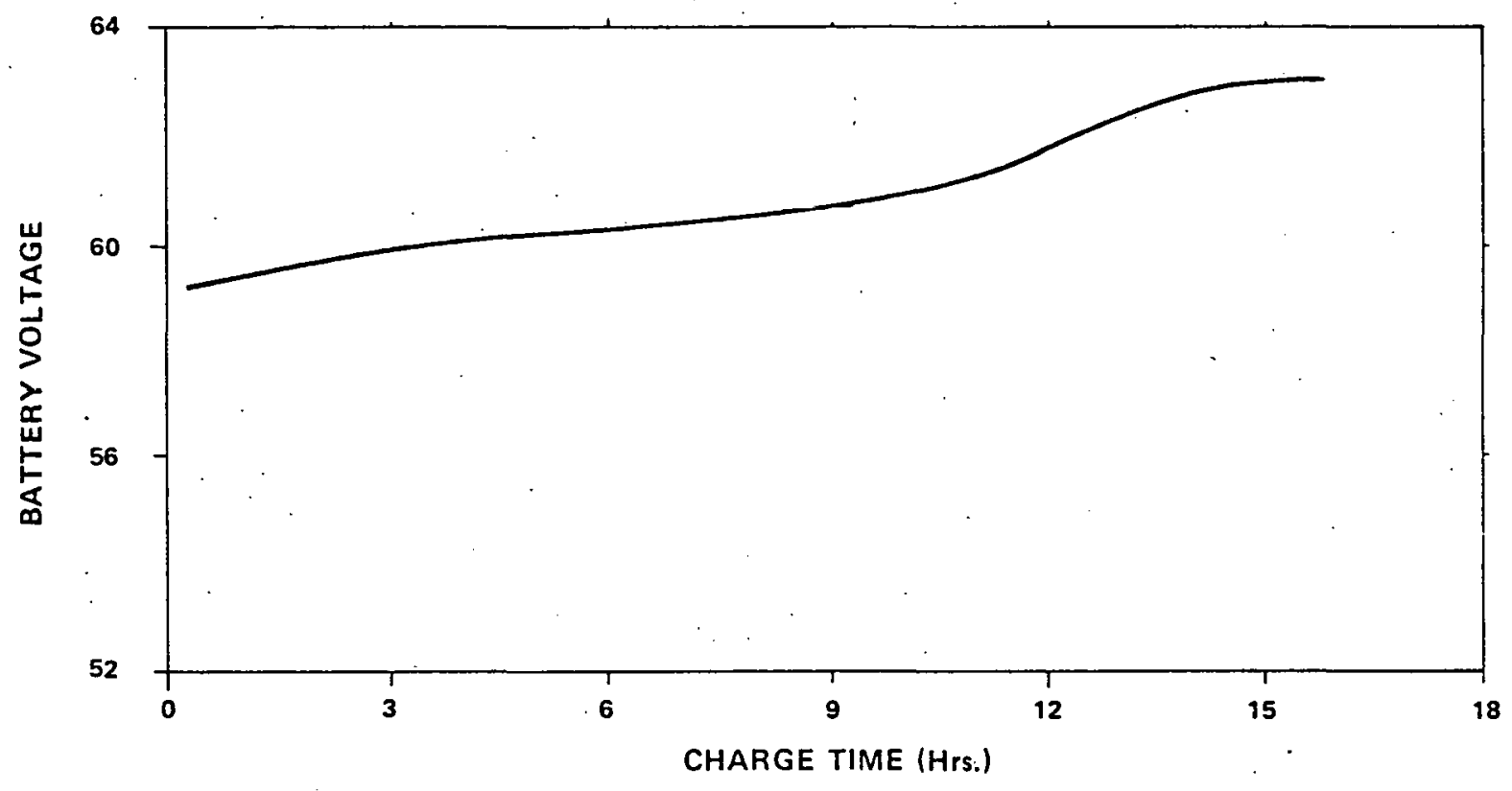

FIGURE 1-32:

BATTERY VOLTAGE AS A FUNCTION OF CHARGE TIME AT THE 10 HOUR RATE. 


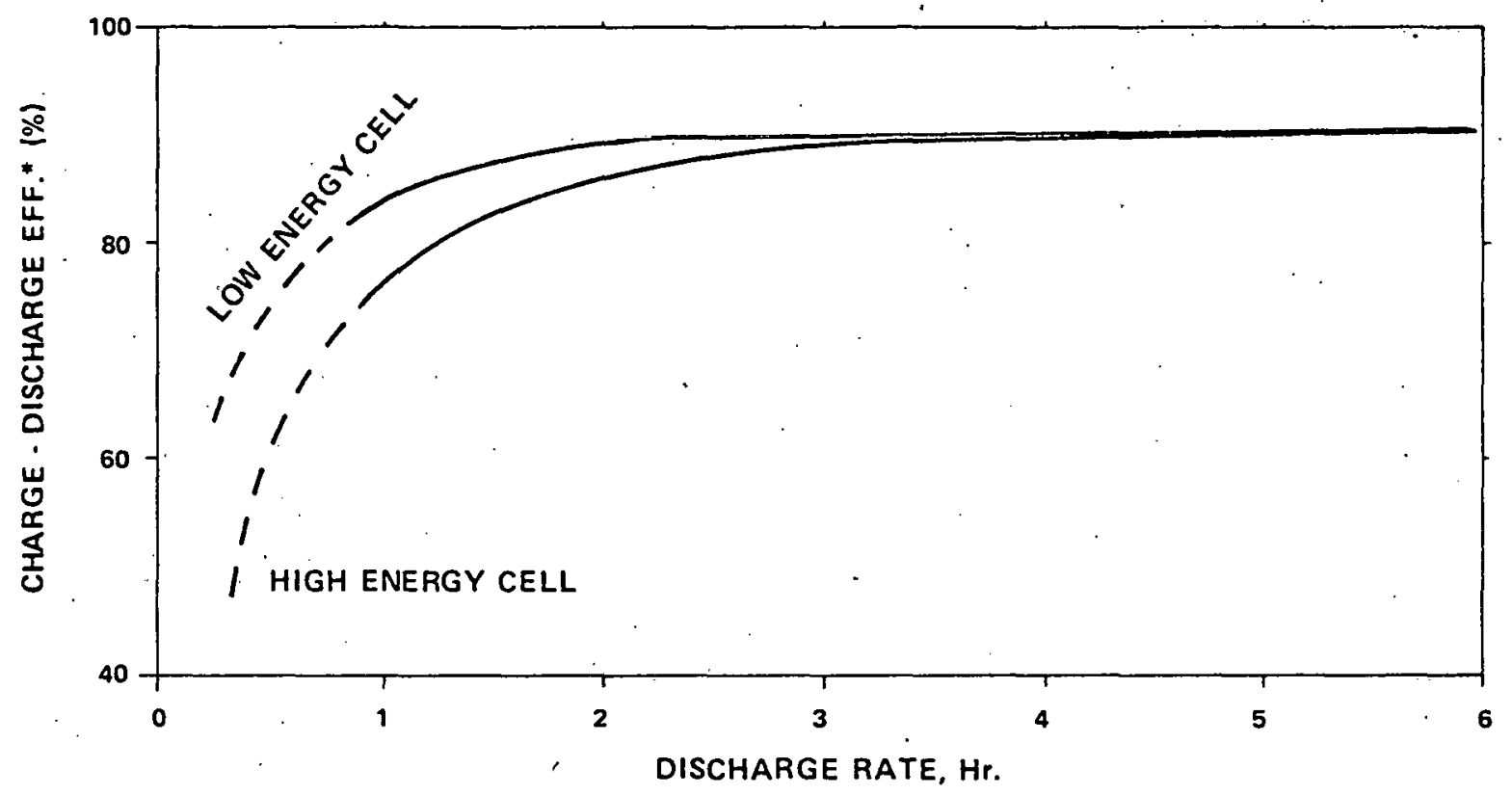

* Assumes Charge Input of $100 \%$ at 12.5 Hour Rate.

FIGURE 1-33:

CHARGE - DISCHARGE EFFICIENCY AS A FUNCTION OF DISCHARGE RATE. 


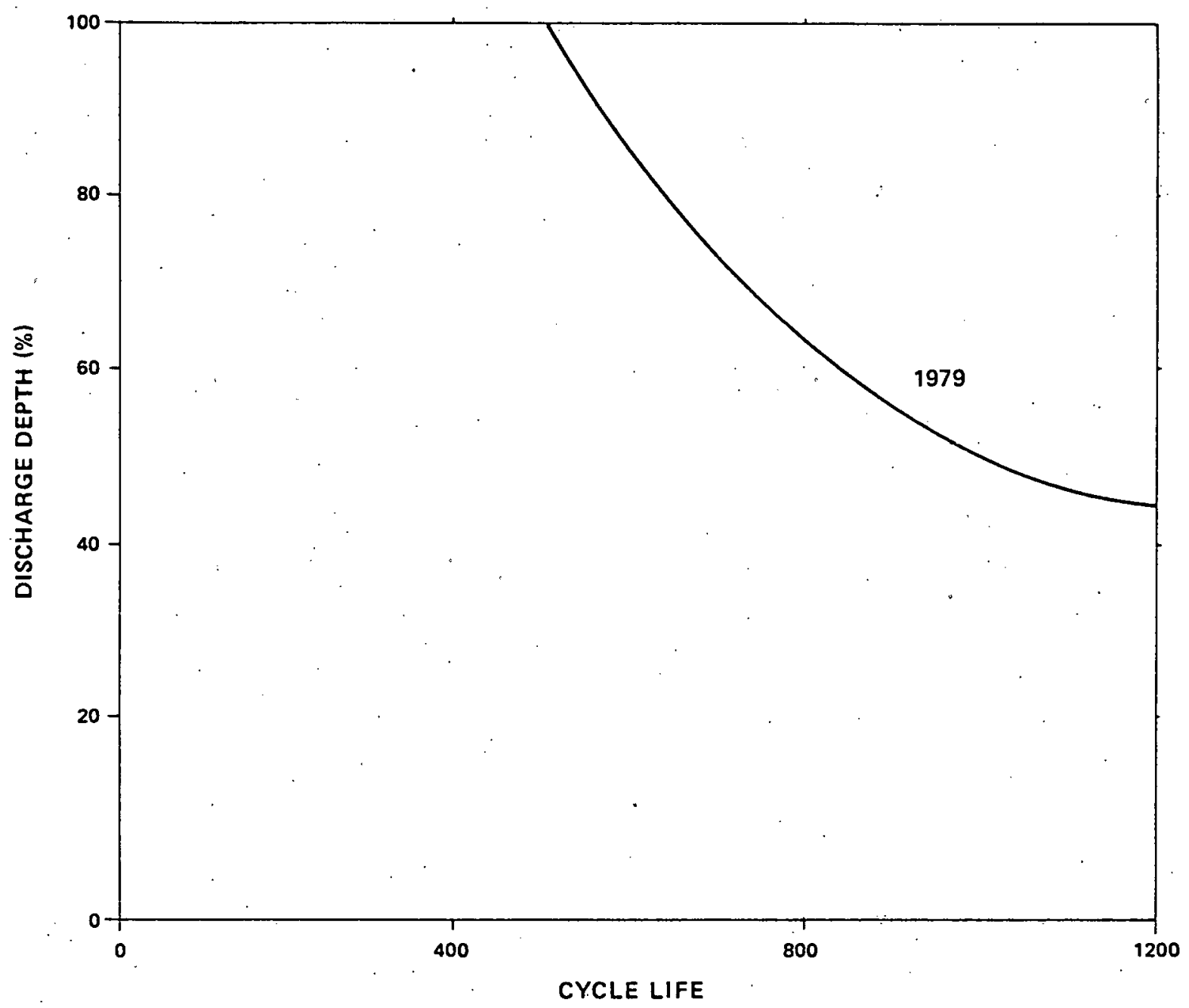

FIGURE 1.34:

PROJECTED CYCLE LIFE (To $70 \%$ Of Original Capacity). 


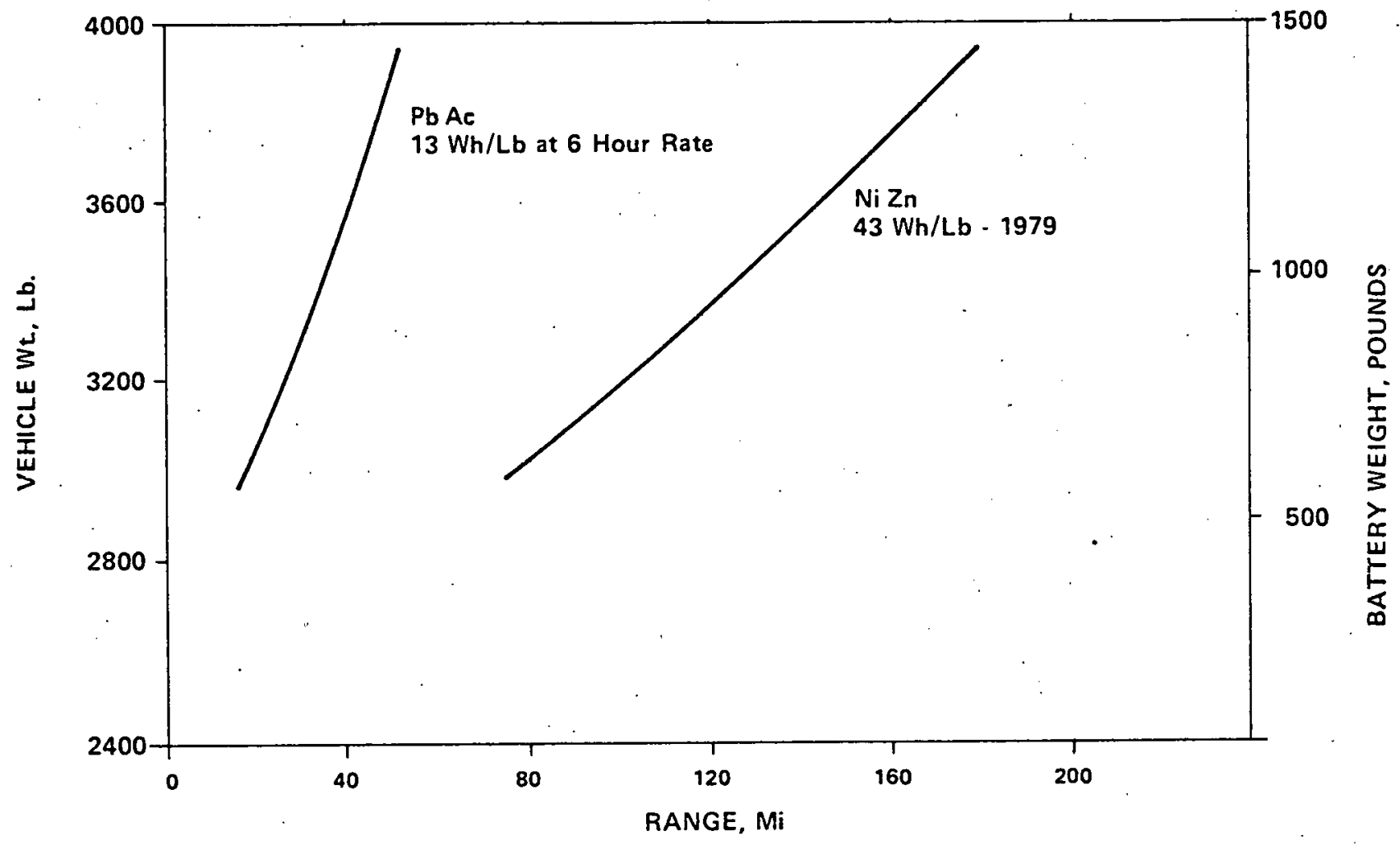

FIGURE 1.35 :

COMPARATIVE BATTERY PERFORMANCE.

Projected Urban Driving Range (SAE Metropolitan Area Driving Cysles) for Compact Cars. $\mathbf{4 5 0}$ Lb. Payload Included. 


\section{CURRENT. DEVELOPMENT PROBLEMS}

\section{A. Problems Related to System Performance Characteristics}

\section{Capacity Decline on Cycling}

As for deep-discharge lead-acid batteries, a feature of the nickel-zinc system's performance which is of greatest concern for on-road electric vehicle applications is the decline in the capacity of a cell (especially high rate capacity) as its cycle life progresses. In actual service, partial rather than complete discharge will be the normal mode of operation and this will retard the actual rate of decline in capacity, particularly at low depths of discharge (e.g., less than 50\%). Such cycling conditions are frequently used by some workers which result in steady capacity over an extended cycle life. It has also been demonstrated that, barring separator failure, the capacity will decline to and level at about $50 \%$ of original capacity. However, vehicle designers are likely to be influenced by performance above 50\% depth of discharge and the decline in capacity from 100 to about $50 \%$ on cycling is a problem that needs further attention if large number of cycles (e.g., $500-1000)$ are to be attained at high energy densities $(35 \mathrm{Wh} / \mathrm{lb}$ or greater).

Cycle life in excess of 500 and even 1000.cycles has been reported in the literature in small nickel-zinc cells, often in cell constructions not always translatable to large electric vehicle cells of higher energy densities. At Gould, it was shown that the scaling up to large battery, as is contemplated for use in certain electric vehicle applications, can cause a substantial reduction in cycle life performance. Consequently, one must exercise extreme caution in projecting cycle life of large cells from small cell technology.

Progress made at Gould on the development of large nickel-zinc cells ( $\sim 400 \mathrm{Ah}$ ) has been encouraging. A cycle life of up to 270 has been demonstrated in first generation cells at an energy density of $30-35 \mathrm{Wh} / \mathrm{lb}$ at the $\mathrm{C} / 5$ rate and approximately $80 \%$ depth of discharge. Although some shape change in the zinc electrode occurred, the primary cause of failure was shorting caused by separator degradation. However, as indicated above, longer cycle life is possible in small cells or even in large electrode tests (e.g., tri-electrode cell configuration using the 400 Ah size electrodes) using the same separator materials. Figure II-1 illustrates the achievements accomplished toward the development of large ( $400 \mathrm{Ah}$ ) cells. In small cells, a cycle life in excess of 700 to about $50 \%$ of original capacity was demonstrated years ago even with cellulosic separator materials (cellophaneVisking) and early type electrodes; but in large (400 Ah) size cells, less than 50 useful cycles were achieved with this type of separation. Based on the improvements made in the overall development of the $400 \mathrm{Ah}$. cell in the last two years, and particularly on the zinc electrode and separator, it is highly probable that the nickel-zinc system can be developed over the next few years to generate large electric vehicle batteries with cycle life in the 500-1000 range at energy densities of 30 to 40 


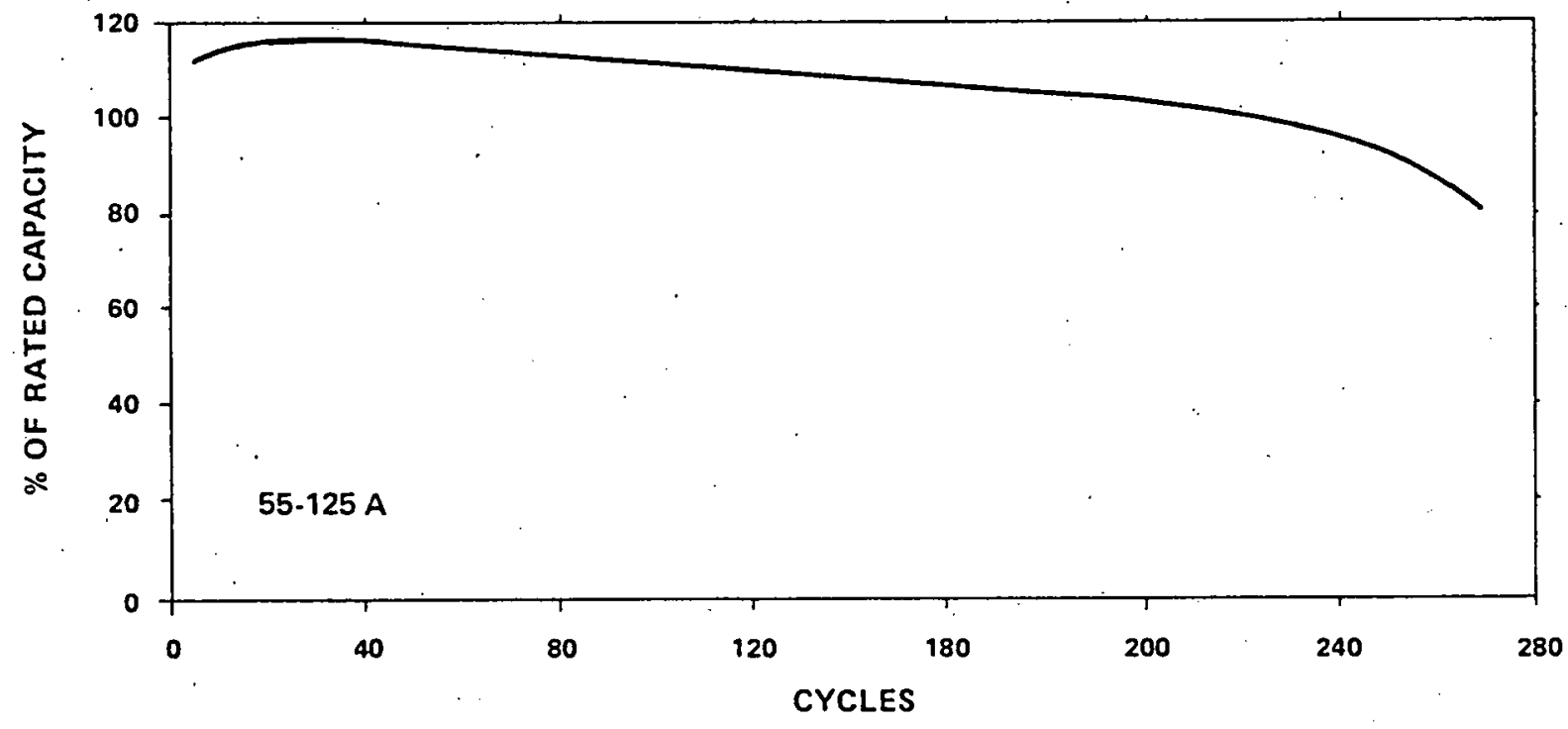

FIGURE |1-1:

CYCLE LIFE OF GENERATION I ( 400 Ah) CELL. 
Wh/lb. It is our opinion that the cycling ability of the large nickel-zinc system is the most important current problem hindering its acceptance in the on-road electric vehicle market.

\section{Electrolyte Maintenance}

Maintenance may also present some problems to users. Like lead-acid batteries, the vented nickel-zinc system will require periodic addition of water to make up losses caused by electrolysis. Any drying out of the battery could present possibilities of fire hazards because of combustion of the zinc in the presence of an oxygen atmosphere in the cell. These problems, if existant, could possibly be eliminated with the development of maintenance-free or low maintenance battery designs. Mechanical means, e.g., automated filling devices, are also available that would simplify the addition of water, thus, making the servicing of vented systems a relatively simple matter.

It should also be noted that shallow discharge regimes, which as previously noted are likely in actual service, promote conditions of cell overcharge and thus increase the frequency of the requirements for water addition. The development of optimum charging techniques should help to minimize overcharging of the cell.

\section{Thermal Characteristics}

A performance feature of the large nickel-zinc system on which insufficient work has yet been carried out is its thermal characteristics. Considerable heat is evolved during continuous high rate discharge, e.g., $500 \mathrm{~A}$. Heat evolution in a large battery, particularly one containing several cells, can cause a substantial rise in internal temperature which can accelerate the degradation of the sep. arators. While in actual electric vehicle service, continuous high rate discharges are unlikely; there can be certain operating applications whereby a significant fraction of the discharge can be at high rates. Consequently, the system must be designed to minimize the heat problem.

Lead-acid traction cells are normally encapsulated in a box. Similar procedures or close packing of cells with little or no allowance for heat dissipation have been used for nickel-zinc by some designers. Encapsulation or similar methods can aggravate any problems deriving from excessive heat evolution within the cell. Gould has given consideration to the heat problem from the inception of the large cell development to its mounting in a battery configuration. Consequently, we do not feel at present that the problem of heat dissipation from a battery pack will be any greater than that in single cells, for which further optimization is likely that could reduce the temperature rise in a cell at high discharge rates. Furthermore, with the development of more stable separators, the effect of temperature on the life of the system should be minimal. 


\section{B. Component Problems}

Our system's decline in capacity as its cycle life progresses are associated largely with such life limiting processes as separator degradation and zinc electrode shape changes. This does not imply, however, that the other major components of the cell, e.g., the nickel electrode, present no problem. On the contrary, it can, depending on the type used, create serious enough problems to warrant some concern. These three problem areas are discussed in greater detail in the paragraphs that follow and their importance assessed.

\section{Separator Materials}

Of all the technical difficulties facing the nickel-zinc system, the life limitation due to the degradation of the separation is the most important problem. Separator materials with the proper physical properties, which are low cost, and possessing sufficient stability in the battery environment, while capable of 500 - 1000 cycles in large electric vehicle batteries, are not commercially available.

As was noted in the previous section on current problems, it is possible to achieve reasonable life in small cells, even with such well known commercial materials as cellophane and Visking sleeve - a long time combination used in the rechargeable silver-zinc cell. This is illustrated in Figure II-2. As can be seen, about 160 useful cycles to $70 \%$ of capacity were demonstrated with cellulosic membranes in early exploratory work at Gould. However, in large electric vehicle cells, the relatively rapid degradation of cellulosic membranes resulted in a cycle life of less than 50 cycles. This is also exemplified by the data shown in Figure II-2.

It was also demonstrated in early exploratory work that the cycle life of small size cells could be significantly increased by the use of a separation made up of commercial materials such as cellophane and polymeric membranes of greater stability than cellulosic types. This is illustrated in Figure II-3.

We have also found that another known commercial membrane separator (Permion by RAI) developed for intended use in the silver-zinc system did not perform satisfactorily in large nickelzinc cells (50 and $400 \mathrm{Ah}$ sizes). On the other hand, another commercial material, Celgard polypropylene film, well known to battery users, was found to perform better than most commercial materials evaluated. Performance of theșe two materials in the nickel-zinc cell is shown in Figure II-4.

Although such a material as the Celgard polypropylene film was shown to perform reasonably well, and although it is probably adequate for a planned cycle life of up to 200 cycles, it seems to lack certain physical parameters necessary for long cycle life (e.g., very fine pores to prevent dendritic growths) in addition to being expensive. A further note should be added with respect to the 


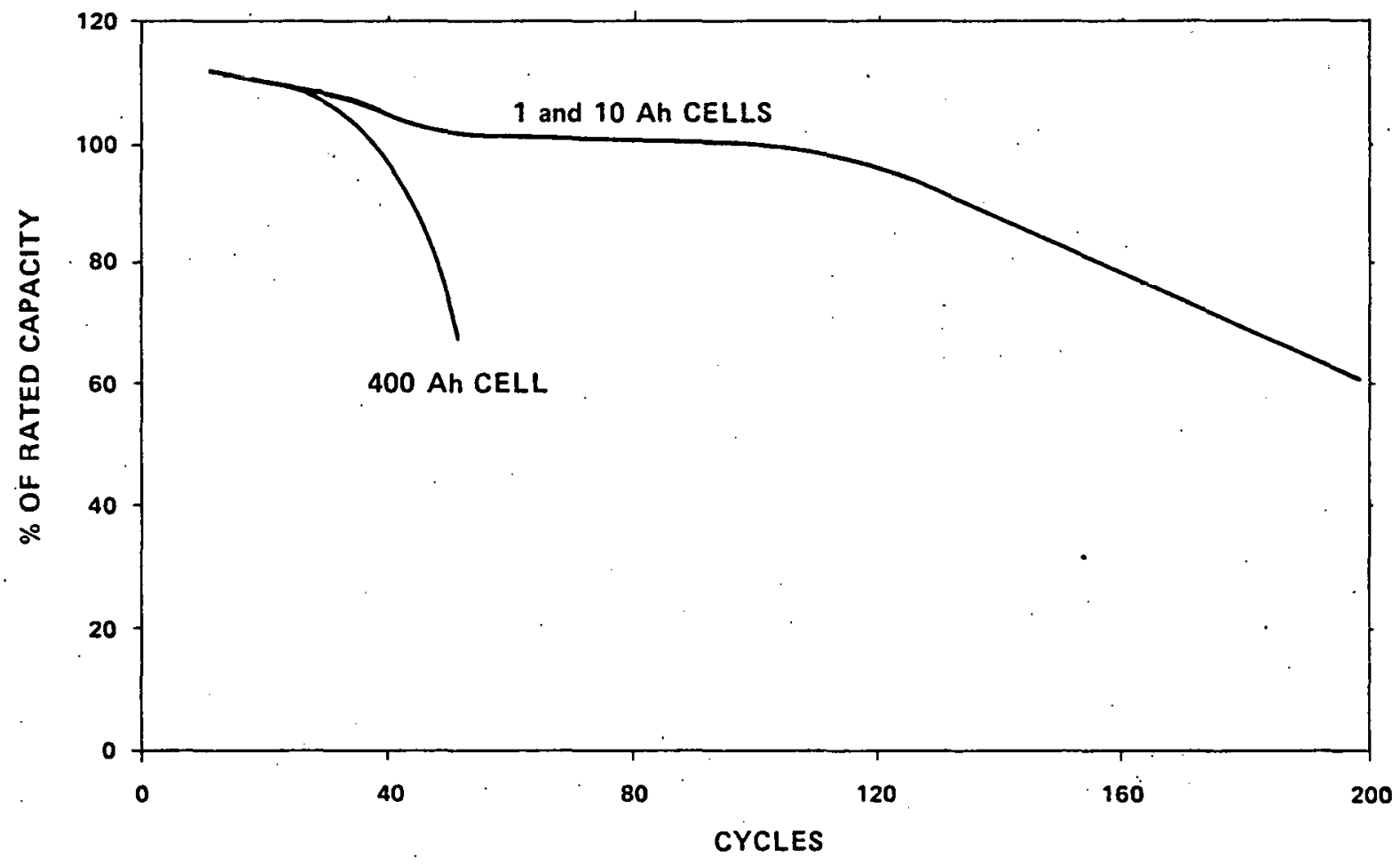

FIGURE 11-2:

CYCLE LIFE OF Ni-Zn CELLS WITH COMMERCIAL SEPARATORS.

Cellulosic (Cellophane - Visking). 


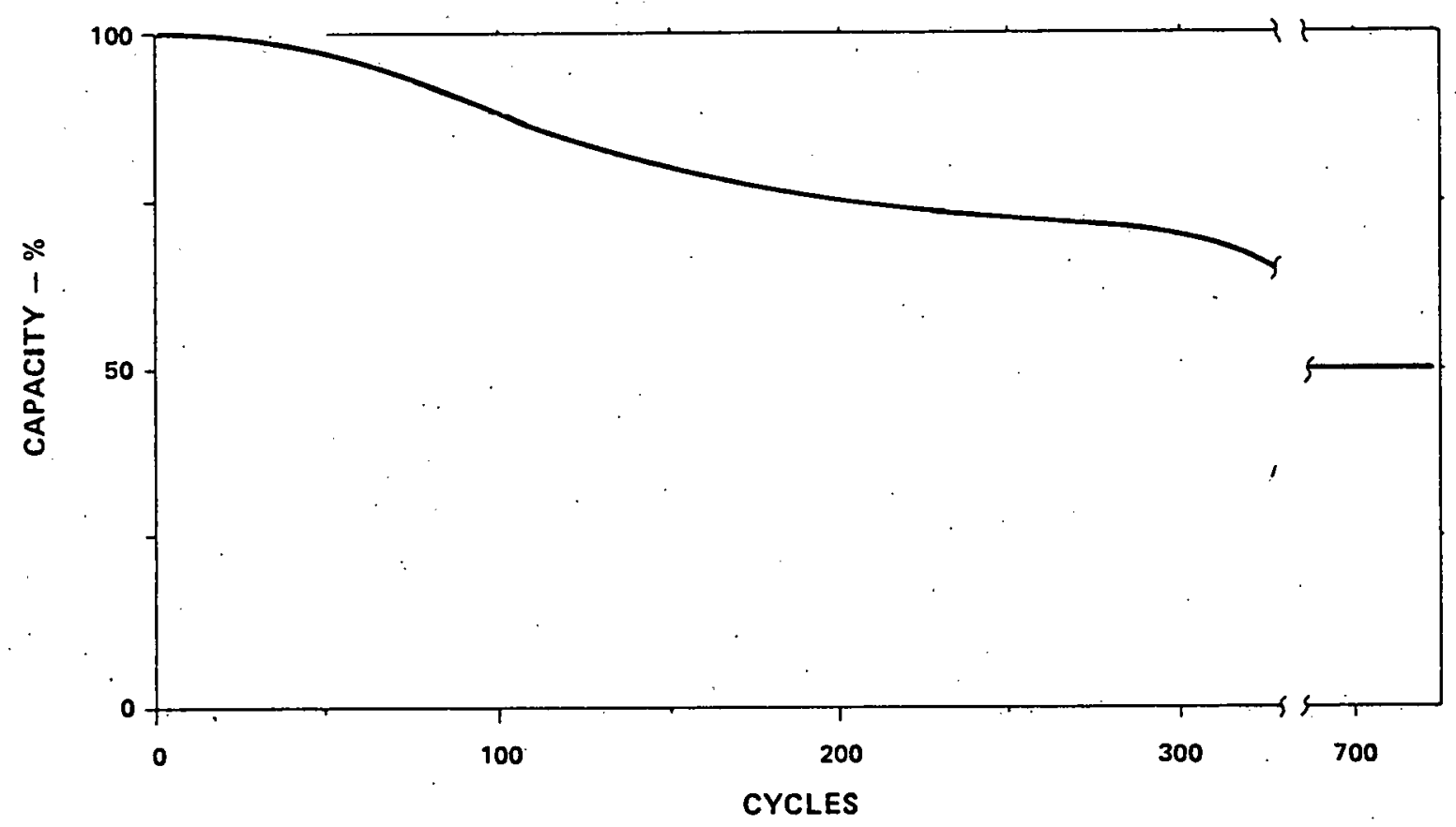

FIGURE II-3:

CYCLE LIFE OF SMALL CELLS WITH COMMERCIAL SEPARATORS. Cellophane - Polymeric Film. 


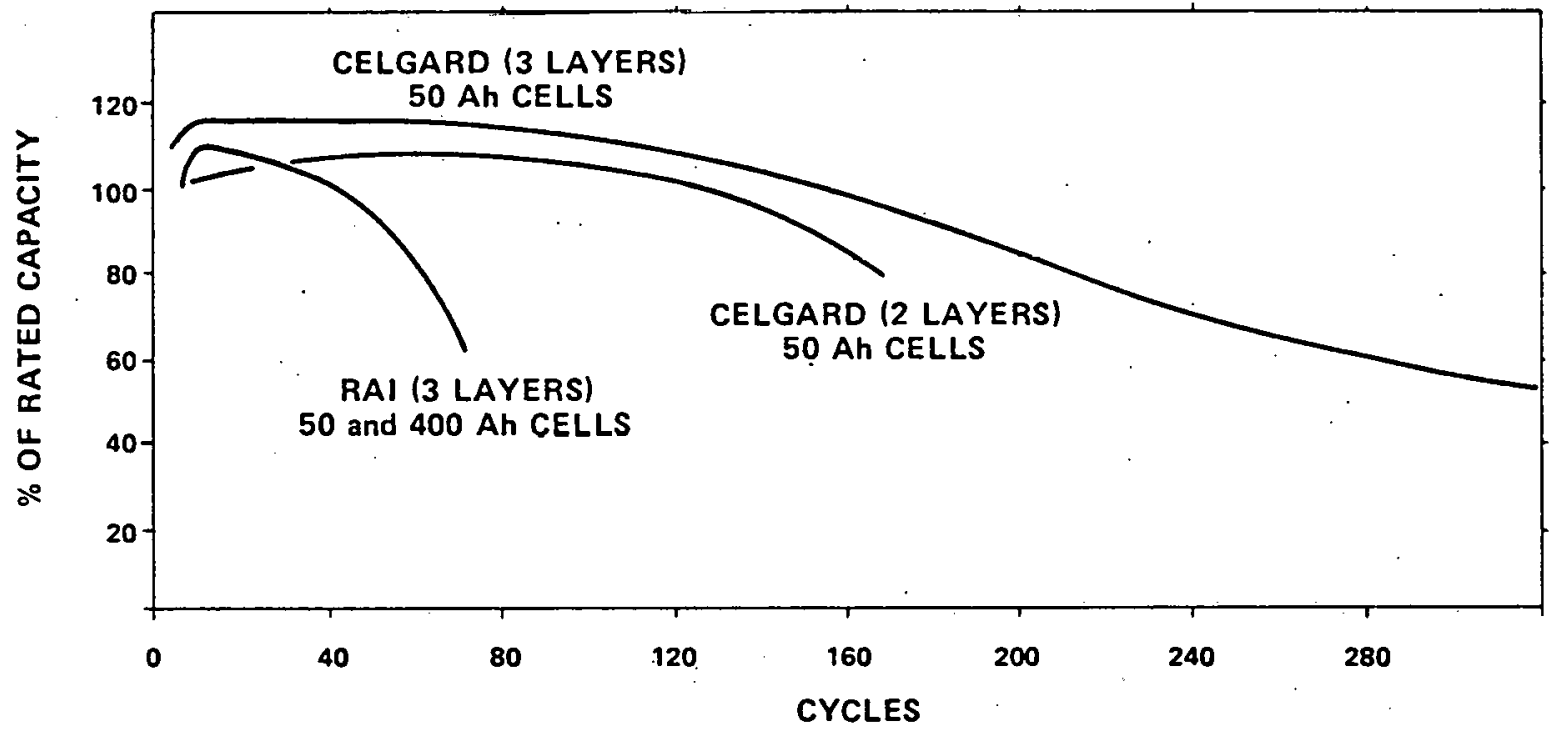

FIGURE |I:4:

CYCLE LIFE OF Ni-Zn CELLS. WITH COMMERCIAL SEPARATORS.

Permion Films (RAI) / Celgard Polypropylene Film (Celanese Plastics Co.). 
use of polypropylene films. Surface treatments of the polypropylene are necessary in order to achieve appropriate wetting characteristics. These generally wear off with time and a slow deterioration in the wettability could prove to be a limitation on the cycle as well as the shelf life of the battery. This deterioration can be accelerated in large size batteries due to higher operating temperatures.

The unavailability of suitable commercial separator materials for the nickel-zinc battery has prompted Gould in recent years to develop its own separators. In this area, Gould is supporting an outside program in addition to its own in-house efforts. The development is directed at both microporous organic membranes and inorganic/organic composite separators for applications requiring modest cycle life (up to 300 cycles) and long cycle life (e.g., 500 or more cycles). Low cost separators, possessing fine pores $(<0.01 \mu)$, low electrical resistivities ( $\sim 10 \mathrm{ohm}-\mathrm{cm}$ ), and which are stable to the cell environment, are being developed.

Progress has been made toward the development of materials that improve the cycle life of large cells. Although the attainment of about 500 useful cycles has yet to be accomplished in large cells, it is our opinion that the controlling factor will be the separator. Based on recent improvements in developing new separators, we feel that the probability of achieving around 500 cycles in large cells over the next few years is relatively high. To achieve cycle life in excess of 500 and up to 1000 cycles may require further advances in the separator in addition to improved zinc electrodes. The discussions that follow are given in support of our optimistic views.

A variety of microporous plastic membranes have been prepared, characterized, and evaluated. A low-cost PVC membrane has been developed that possesses the fine pores and low electrical resistivity needed to prevent dendrites and yield satisfactory performance characteristics. However, this membrane is not as stable as desired in the large cell environment. Despite this drawback, the PVC membrane, in combination with Celgard, delivered up to 270 useful cycles in the large 400 Ah cell (Figure II-1) which is better than any commercial material evaluated. These results suggest that a material stable to the cell environment and possessing the fine pore properties and low resistivity of the above membrane could possibly significantly increase the cycle life of the large cell while retaining a satisfactory energy density.

Progress is currently being made toward the development of such plastic membranes. For example, a nylon membrane has been developed that is potentially more stable than PVC in the large cell environment. Although this material did not possess the very fine pore measured with the PVC type, it has thus far performed satisfactorily in large 400 Ah cells (Figures II-5 and II-6). As for the PVC membrane, the nylon is combined with Celgard film in these cells. Recent process development efforts have produced a nylon membrane with finer pores which appears to prevent dendrites. Secondary electron images (SEI) of cross-sections of virgin films are shown in Figure II-7. 


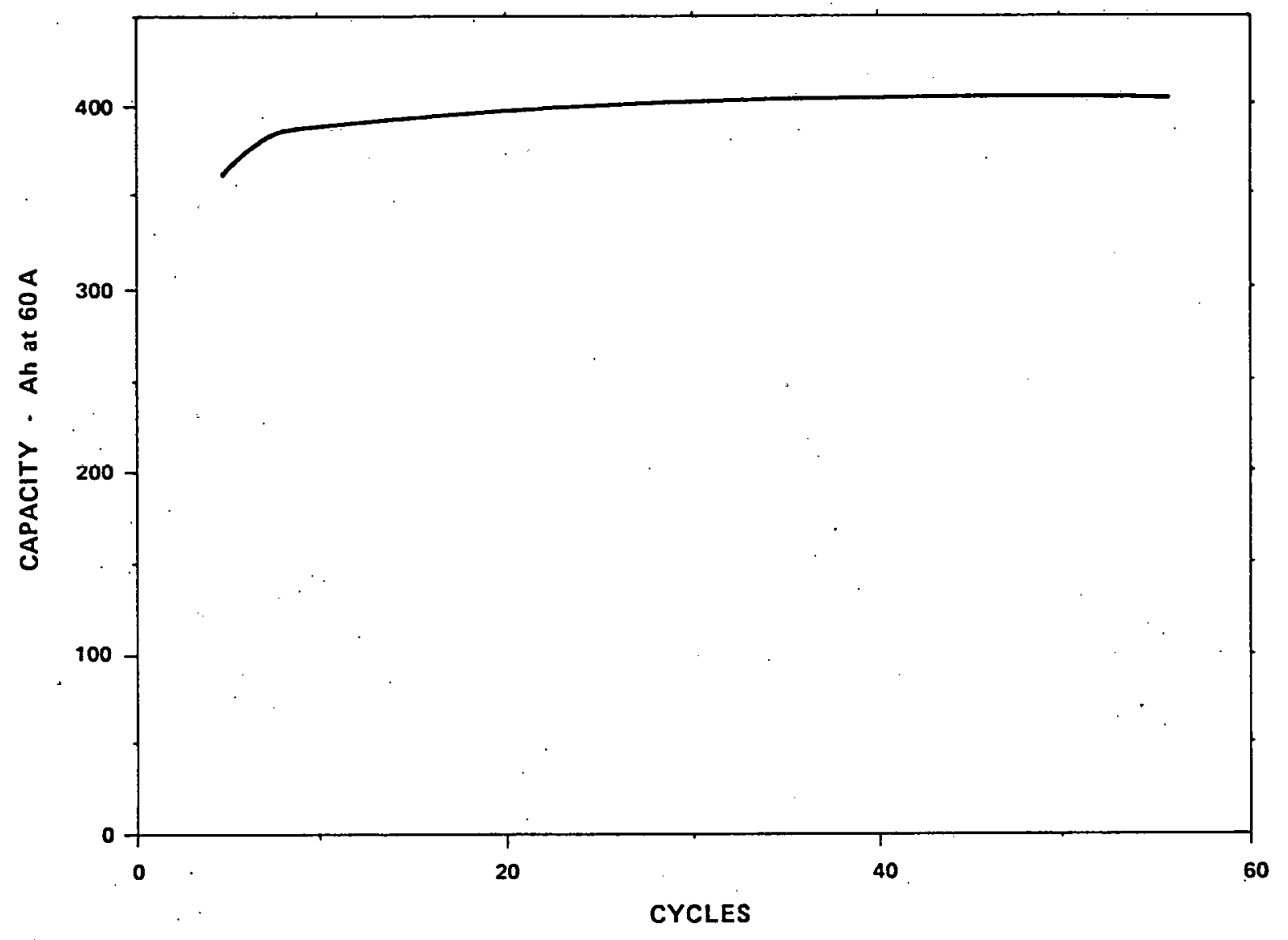

FIGURE 11-5:

CYCLE LIFE OF GENERATION I ( 400 Ah) CELL WITH NYLON-CELGARD SEPARATION. 


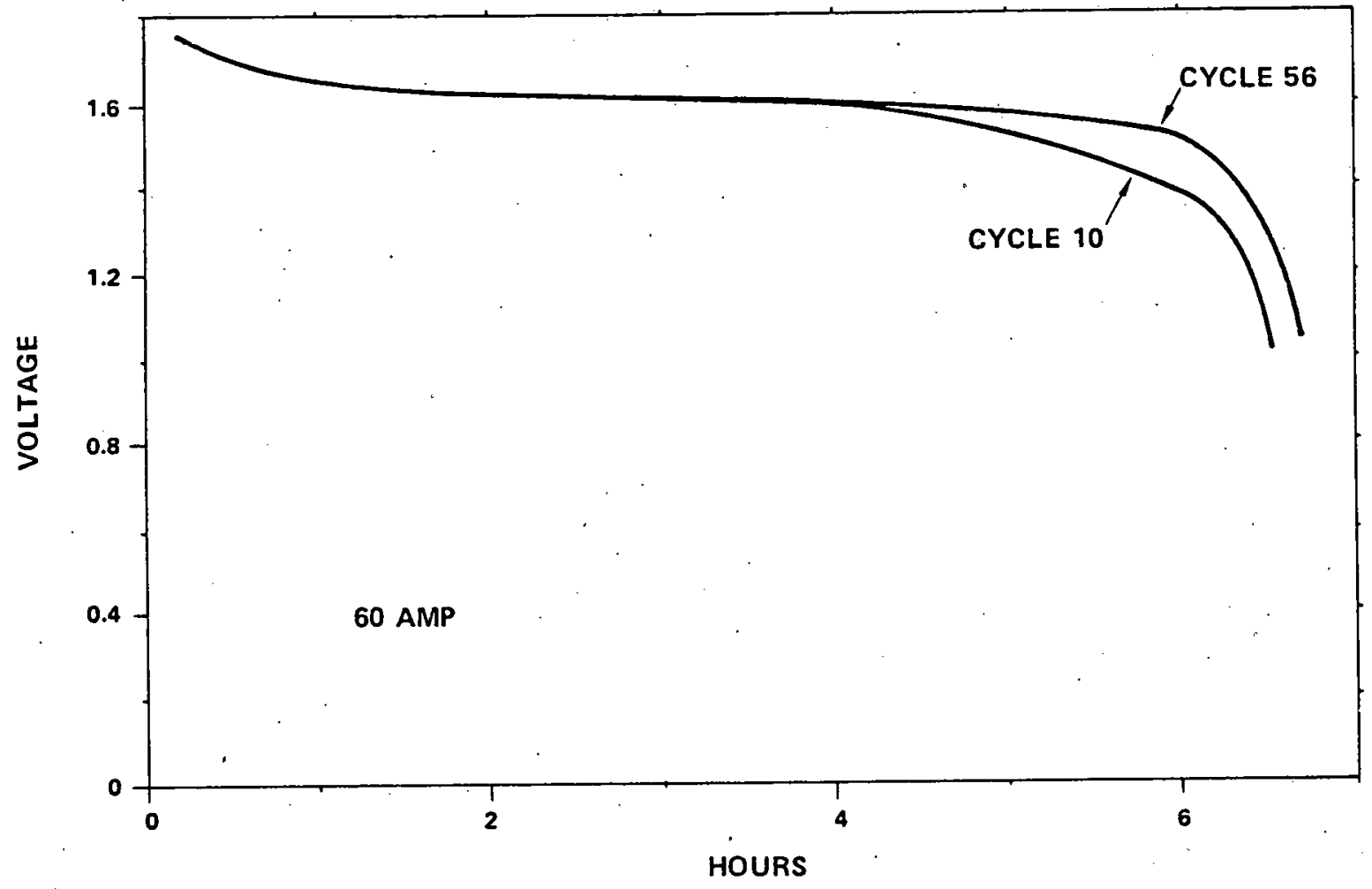

FIGURE 1I-6:

DISCHARGE CHARACTERISTIC OF GENERATION I ( 400 Ah) CELL WITH NYLON-CELGARD SEPARATION. 


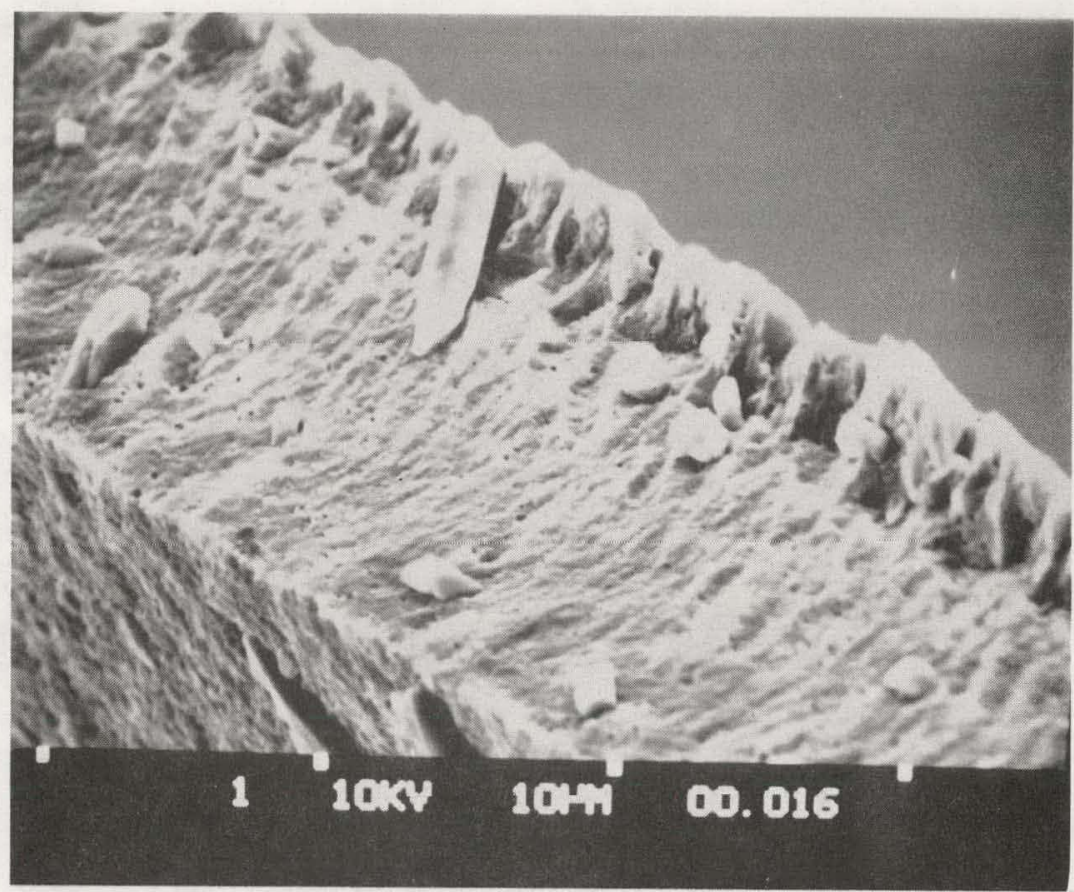

70-50

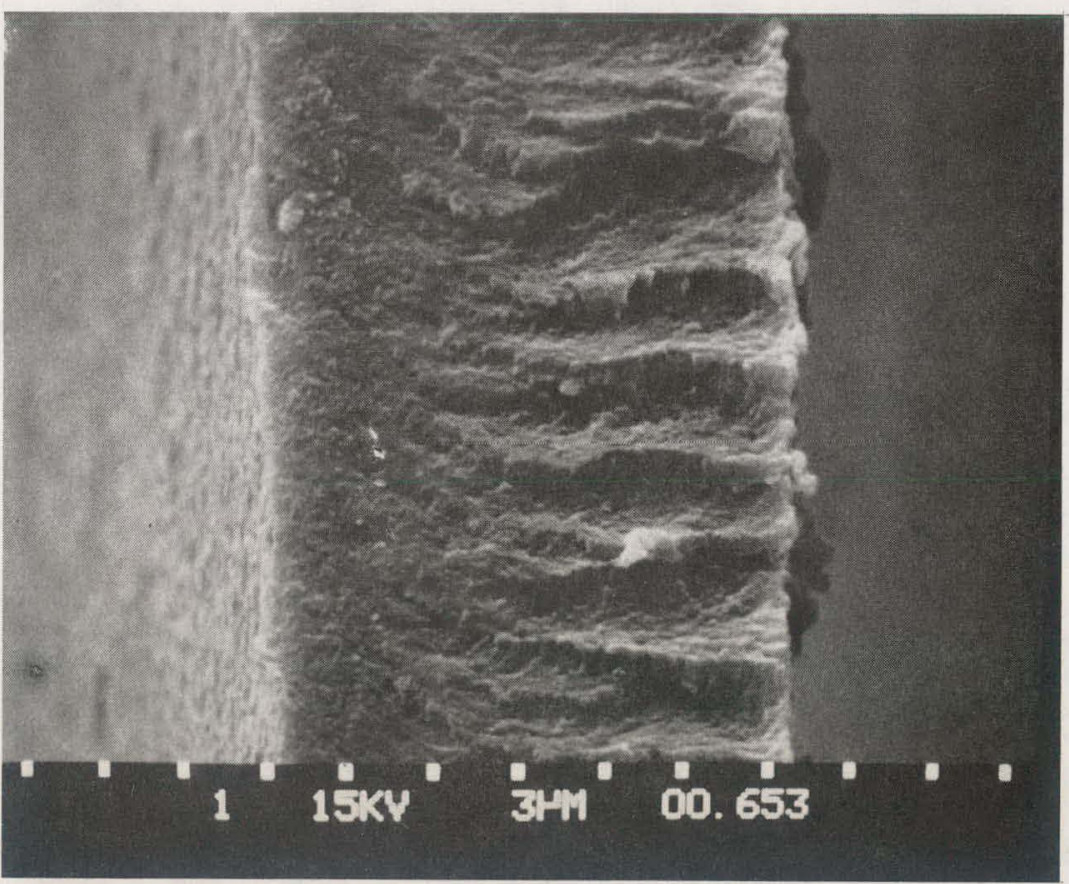

72-1
FIGURE II-7:

SECONDARY ELECTRON IMAGES OF CROSS-SECTIONS OF VIRGIN NYLON FILMS.

Recent Material With Finer Pores. $3000 \mathrm{X}$ 
As can be seen, the films are highly dense yet microporous. For comparison, the cross-section of a film of a totally different structure and representative of the earlier nylon films is shown in Figure II-8. Secondary electron images (SEI) of both sides of this film are also shown in Figure II-9. This type has an open sponge-like structure with a skin whose porosity differs from the bulk porosity and was not as effective as a barrier for dendrites. Figure II-10a is an SEI of the crosssection of a double layer of film of the sponge-like structure after tested in cell and Figure II-10b is an elemental $\mathrm{x}$-ray map of zinc. Penetration of zinc was through both layers. In contrast, the recent nylon film contained no zinc deposits after testing in cells (see Figures II-11a, 11b and 11c).

On the basis of the data contained herein, it can be concluded that materials can be produced with physical characteristics that are effective in preventing zinc dendrite growth, while performing satisfactorily as a separation. A drawback with the nylon film so far is its insufficient chemical stability in the cell environment.

In addition to the above mentioned materials, microporous films have been made recently from at least one other polymeric material that appear to be far more chemically stable than either PVC or nylon, beside possessing reasonably fine pores and low resistivity. The processes for producing this material on a larger scale required for evaluation in large cells are currently being developed.

In the area of composite separators (inorganic/organic types), Gould is developing low cost materials that provide the required porosity and conductivity, possess small pore size, are chemically stable, and mechanically strong. Such materials offer promise in terms of long calendar and cycle life. A first version of these materials which was produced continuously is currently being evaluated in $400 \mathrm{Ah}$ size cells, and 12 volts - $40 \mathrm{Ah}$ and $60 \mathrm{Ah}$ batteries. When used in combination with the Celgard polypropylene film, this composite material exhibits the performance characteristics shown in Figure II-12 for a 40 Ah, 12 volt battery. Most importantly, this separator has held up very well from a chemical and thermal stability standpoint in one hour rate testing to date.

Current development efforts on the composite separator are concentrated on refining the continuous processes to produce more uniform materials with finer pore size and lower resistivities.

The physical properties for typical commercial materials and key Gould developed materials are compared in Table II-1. The data shows that progress is being made toward developing separator materials with fine pores and low resistivities. Included for reference purposes are the recently reported data (Ref. 3 ) on NASA and ERC's separators. Gould will evaluate the NASA separator in the next few months as part of our submersible battery contract with the Navy Underwater Systems Command.

The chemical instability of the separator in the cell environment has to be rated as one of the more serious separation problems causing cell failure. However, premature cell failure due to shorting can generally be associated with flaws or non-uniformity in the separator. This problem 


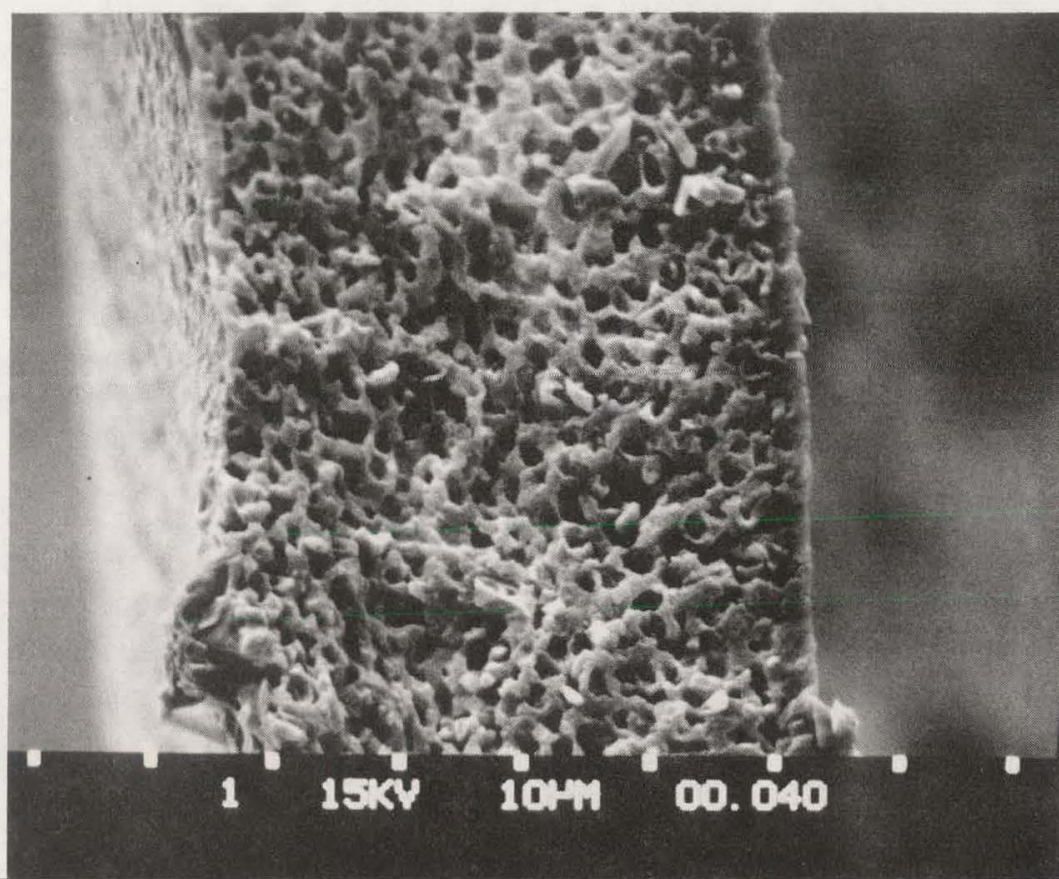

FIGURE II-8:

SECONDARY ELECTRON IMAGE OF CROSS-SECTION OF VIRGIN NYLON FILM.

Earlier Material With Larger Pores. $1400 \mathrm{X}$

$66-9$ 


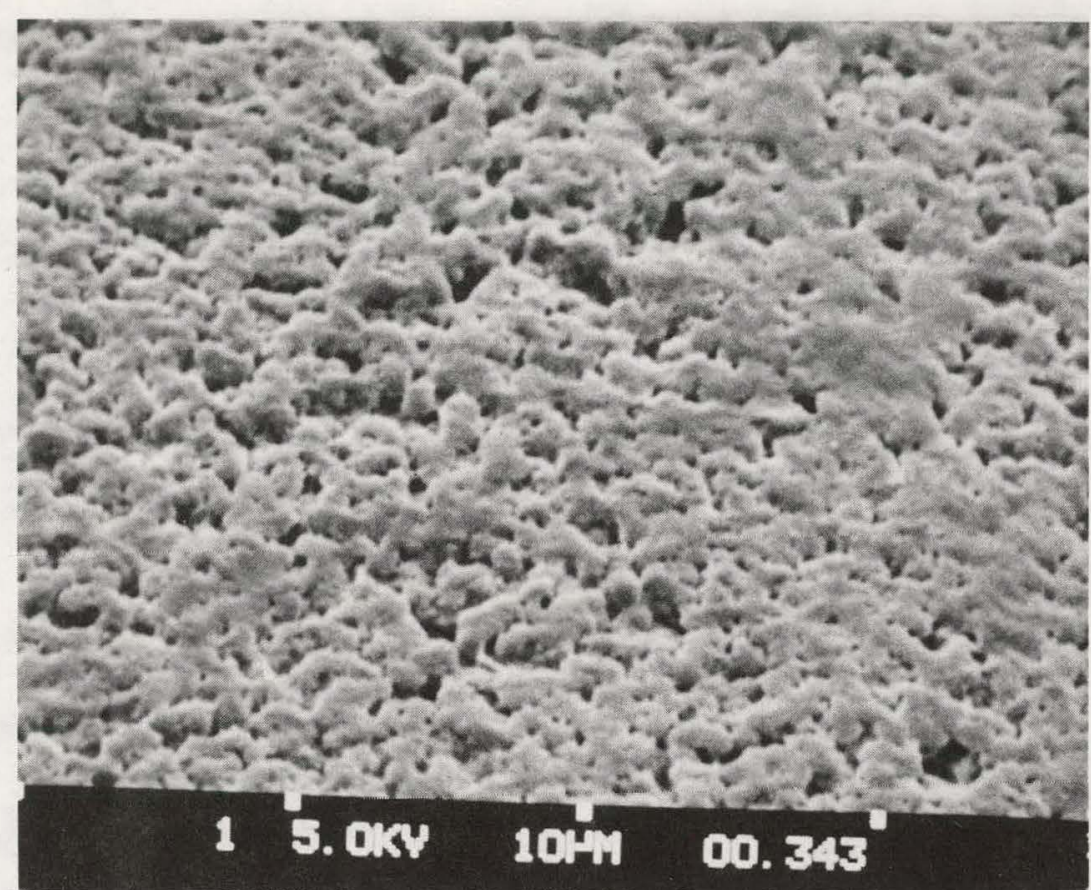

$66-9$
FIGURE II-9:

SECONDARY ELECTRON IMAGES OF VIRGIN NYLON FILM.

Earlier Material With Larger Pores. $3000 \mathrm{X}$

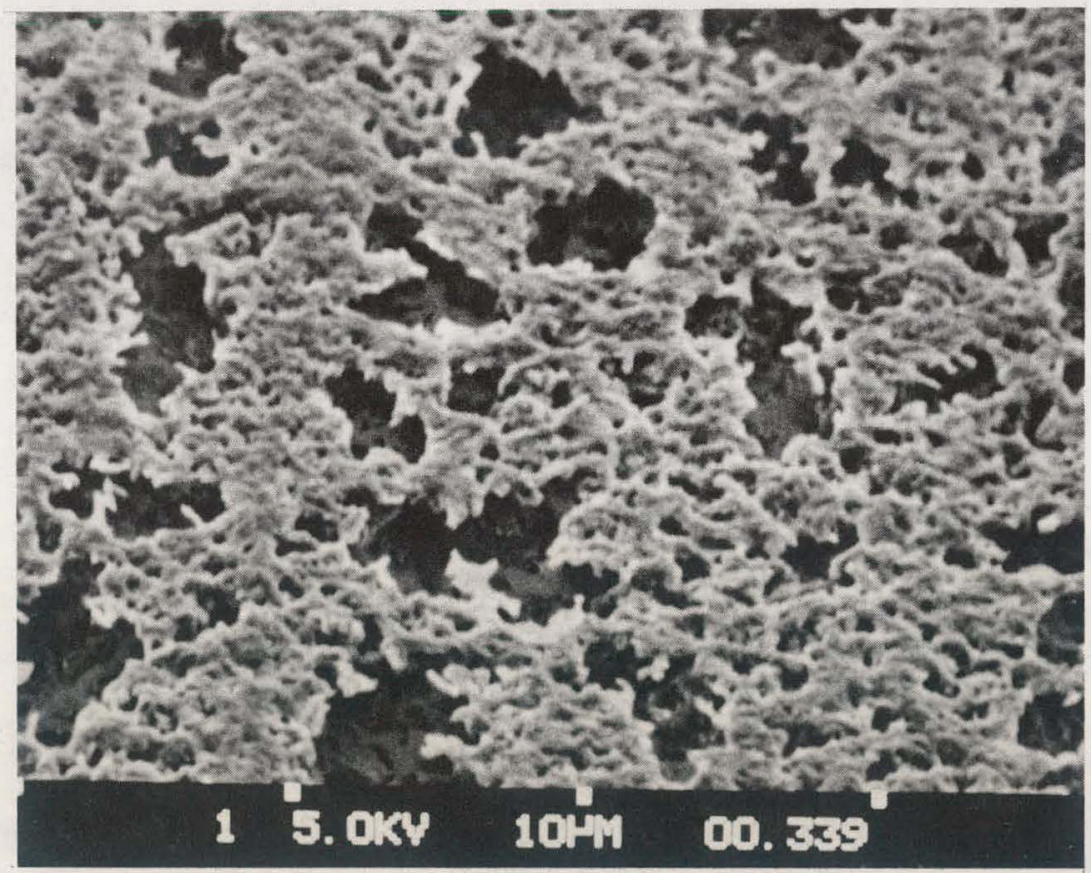

66-9 


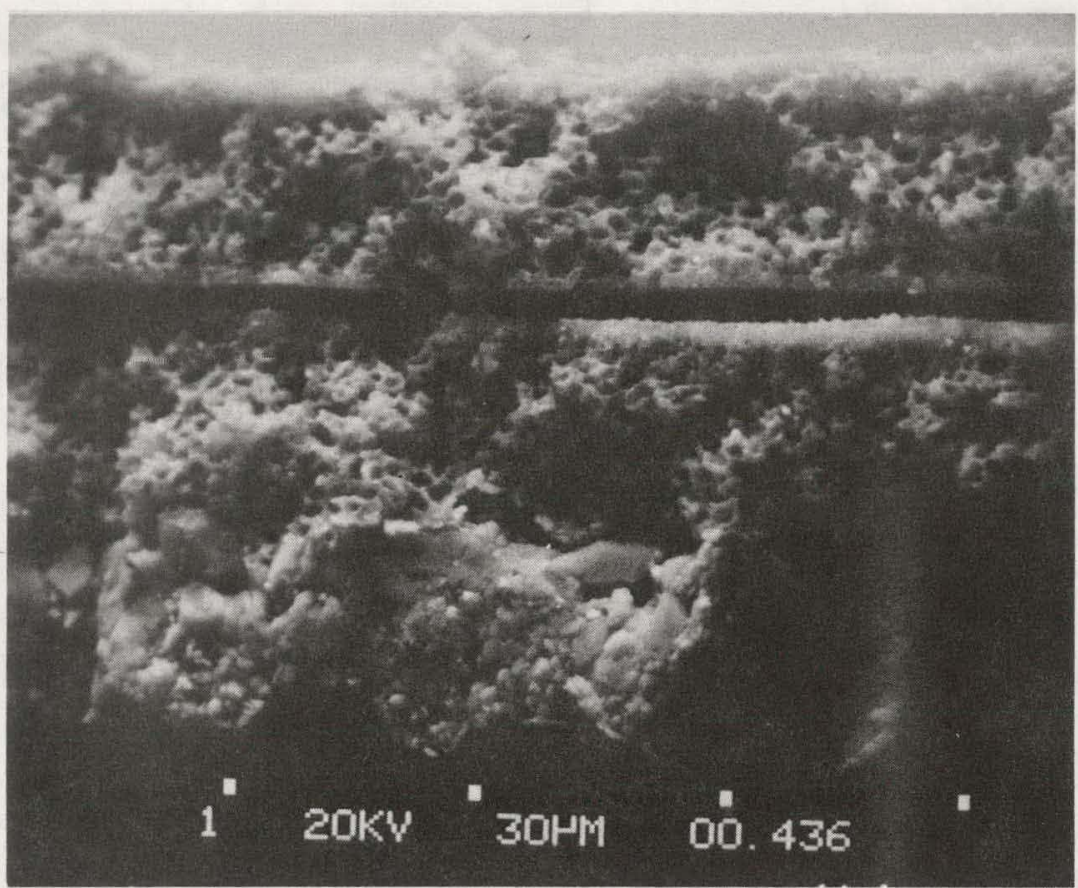

$\mathrm{Ni}$

FIGURE II-10a:

SECONDARY ELECTRON IMAGE OF NYLON FILM AFTER CELL TESTING. Earlier Material With Larger Pores. $850 \mathrm{X}$

Zn

66-15

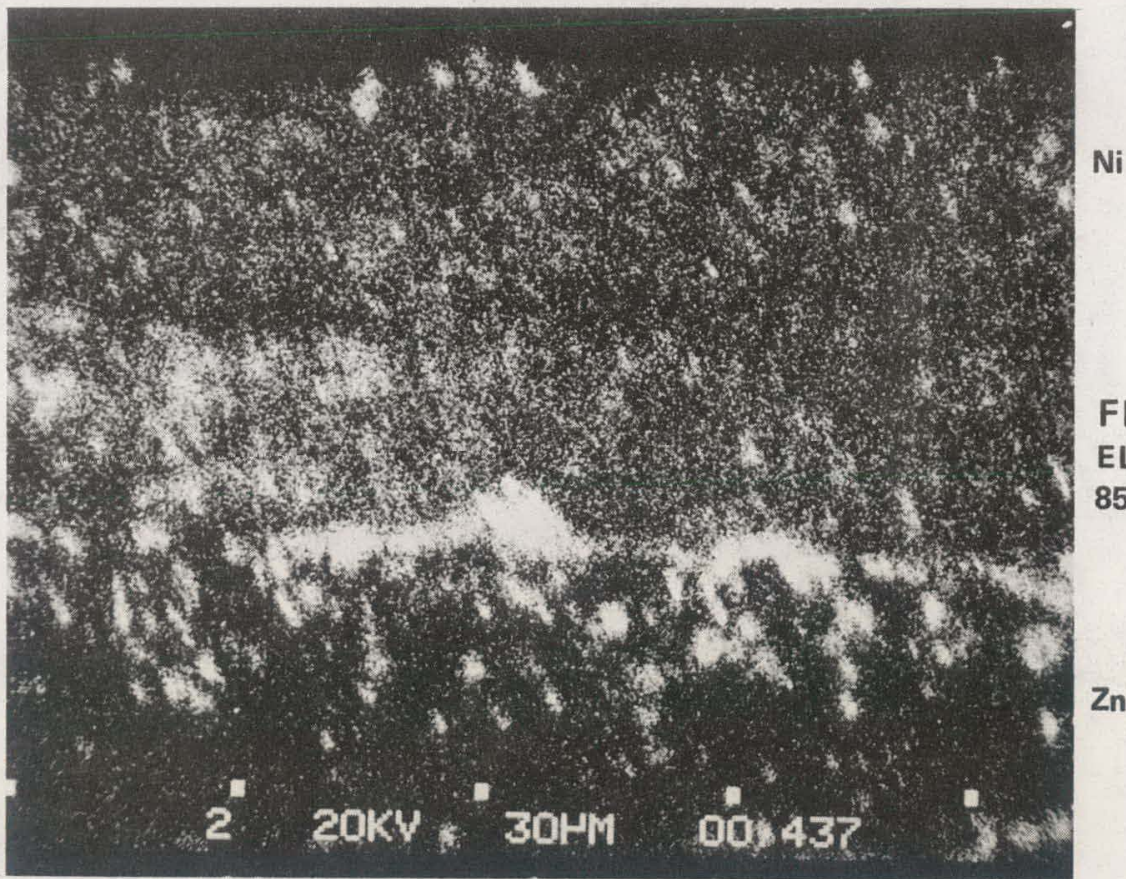

66-15

FIGURE II-10b:

ELEMENTAL X-RAY MAP OF $\mathrm{Zn}$. $850 X$

$\mathrm{Zn}$ 


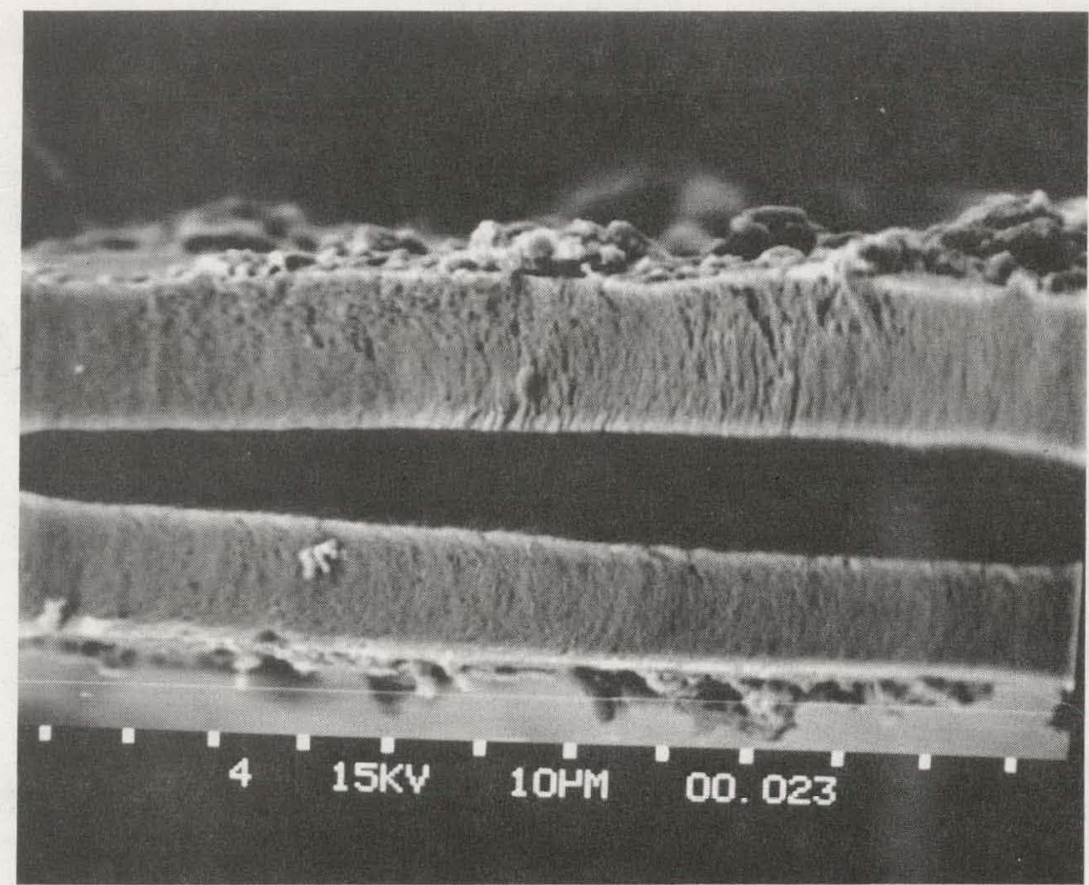

$\mathrm{Ni}$

FIGURE II-11a:

SECONDARY ELECTRON IMAGE OF NYLON FILM AFTER CELL TESTING.

Recent Material With Finer Pores. $1000 \mathrm{X}$

Zn

70-50

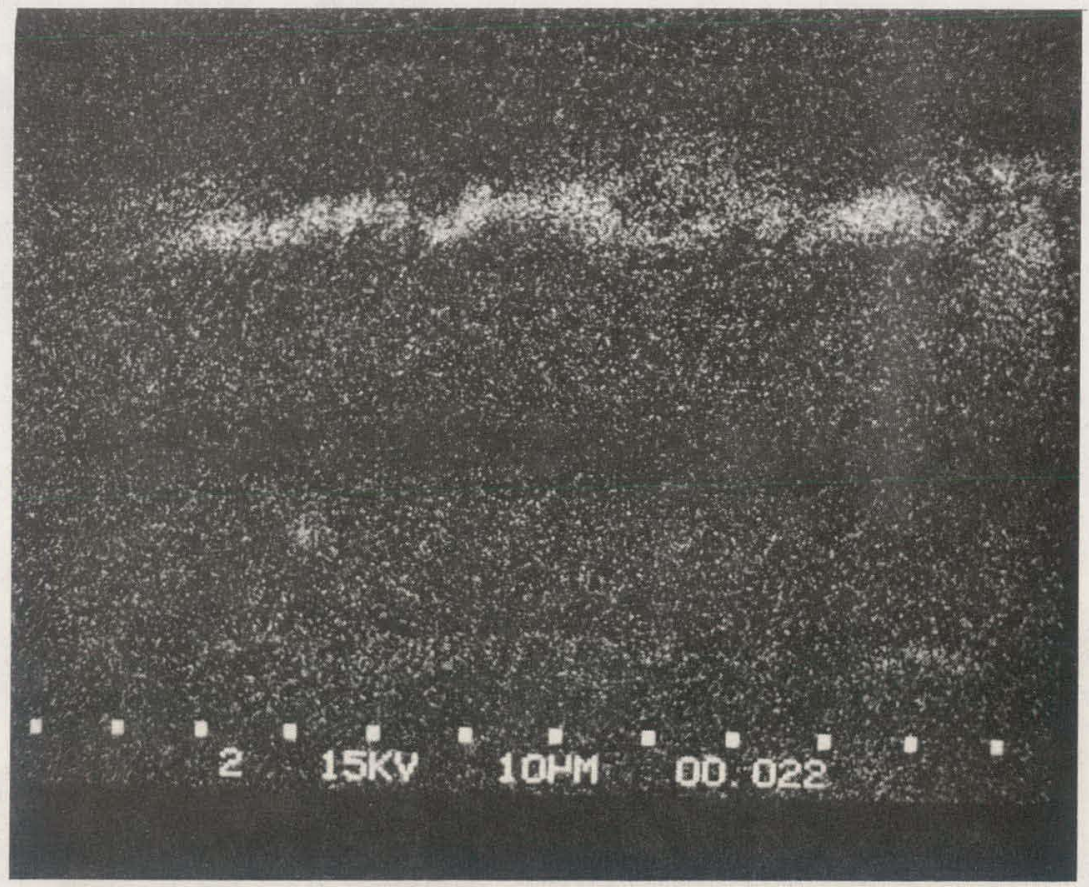

FIGURE II-11b:

ELEMENTAL X-RAY MAP OF $\mathrm{Zn}$. $1000 \mathrm{X}$ 


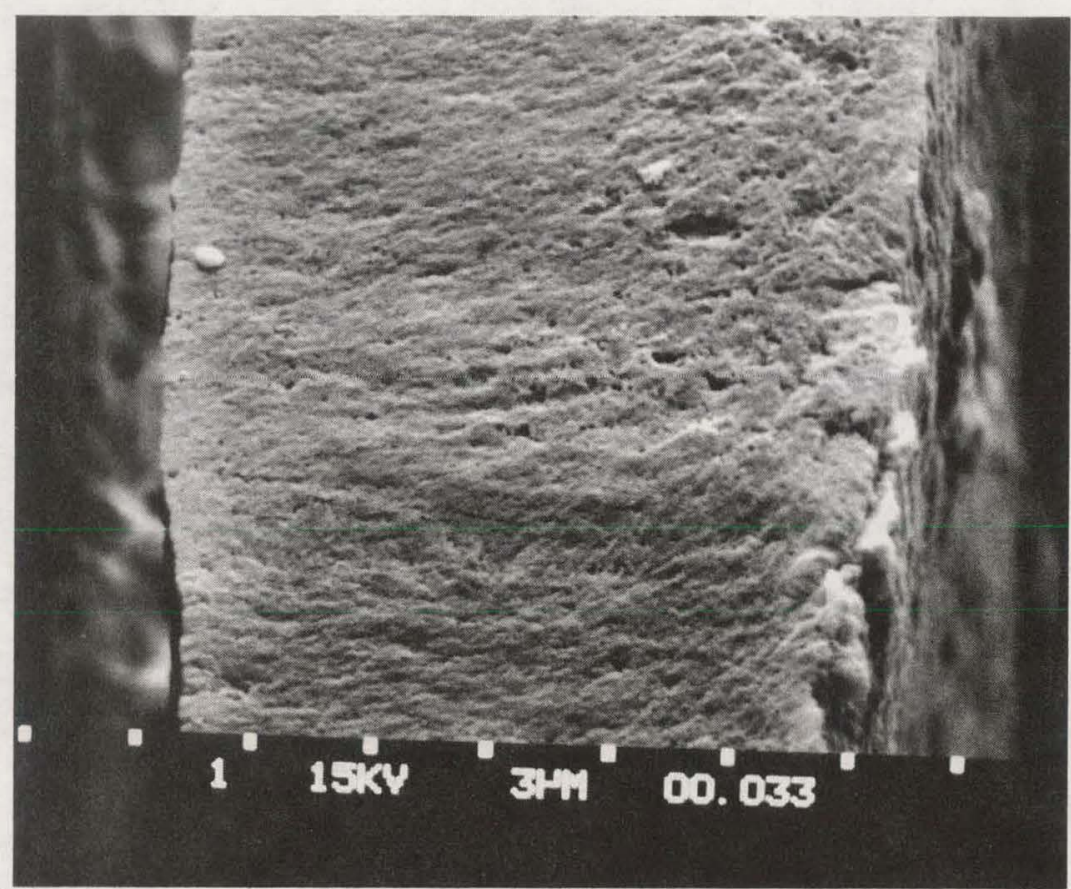

70-50
FIGURE II-11c:

SECONDARY ELECTRON IMAGE OF NYLON FILM AFTER CELL TESTING. Recent Material With Finer Pores. 4500X 


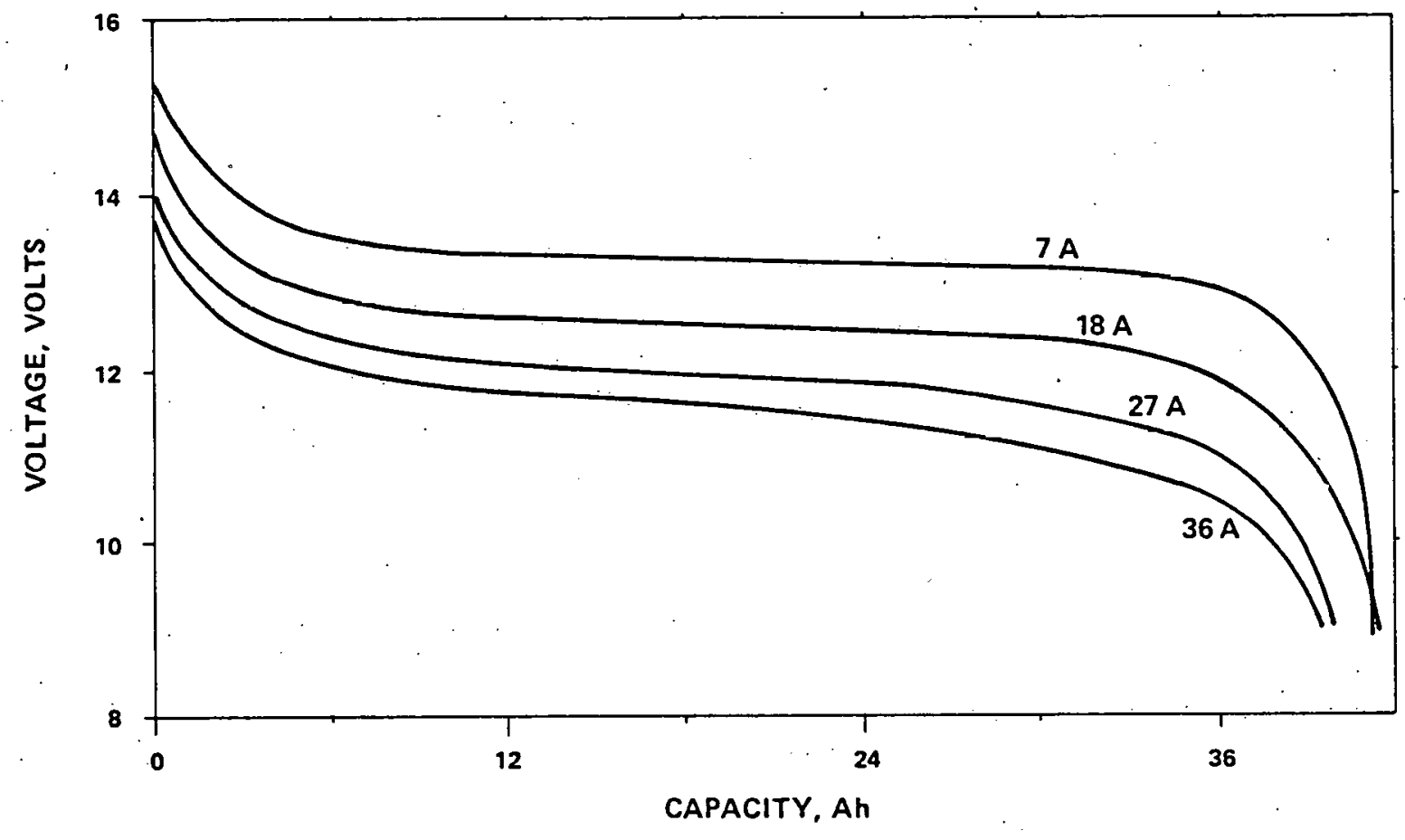

FIGURE 11-12:

PERFORMANCE OF 40 Ah, 12-VOLT BATTERY WITH GOULD

I/O-CELGARD SEPARATION. 
TABLE ||$-1$

PHYSICAL PROPERTIES OF TYPICAL SEPARATOR MATERIALS

MEAN PORE SIZE, MICRON

COMMERCIAL MATERIALS:

CELLOPHANE

VISKING

CELGARD $\ll 0.01$

$\ll 0.01$

0.11
REȘISTIVITY, ohm-cm

$8-14$

7

$1 \cdot 2723^{(1)}$

GOULD DEVELOPED MATERIALS:

MICROPOROUS PLASTICS:

PVC

NYLON TYPE A

NYLON TYPE B

RECENT MATERIALS

INORGANIC/ORGANIC COMPOSITE

$$
<0.01
$$

0.18

$0.005 \cdot 0.04$

0.0017

$0.010 \cdot 0.013$
$10 \cdot 30$

38

$4-34$

$12 \cdot 18$

$24 \cdot 68$

\section{OUTSIDE DEVELOPED MATERIALS:}

ERC 2002

$0.015^{*}$

$16 *$

NASA/LEWIS $1 / 0$

$0.045^{*}$

$16^{*}$

GOALS:

$<0.01$

$\sim 10$

(1) Typical 15 to 100 ; valves dependent on storage and soak time.

- See Reference 3. 
is also one that needs full attention. It is our opinion that this problem can be solved if given . sufficient time and effort to improve the process.

A great deal of emphasis has been placed on the separator problems in recent years. Based on our achievements to date, it seems reasonable to presume that the current separator programs will advance the state-of-the-art from both technical and economic standpoints.

\section{Zinc Electrode}

Although the separator plays, at present, a very key role in the life limitation of the nickelzinc system, the problems associated with the zinc electrode are of no lesser importance if a cycle life of up to 1000 cycles is to be achieved in large batteries in the years ahead, particularly at the kind of performance expected (e.g., 1000 cycles to about $70 \%$ of original capacity and initial energy density of about $35 \mathrm{Wh} / \mathrm{lb}$ ).

The two major problems, which are electrochemical in nature, are:

- Erosion of zinc electrode along the edges on cycling, e.g., shape change and its associated capacity loss (non-uniform re-distribution of zinc throughout the electrode).

- Dendritic growths through the separator of zinc species in the electrolyte; dendrites can form through either flaws in the separator or following deterioration of the separator in the cell environment.

In addition to the electrochemical problems, grid design also affects zinc electrode performance and should be studied further.

Problems associated with the manufacturing of zinc electrodes include the following:

- Mechanical strength of large electrodes for handling under manufacturing conditions.

- Grid design suitable for manufacture and handling while satisfying the requirements imposed in the operation of the battery - both electrical and electrochemical.

- Uniform distribution of zinc mass within any portion of the electrode.

- Use of binders in the zinc mass needed for strength and/or additives needed for cycle life that are acceptable from both cost and performance standpoints. Any additives, other than binders, must also be adaptable to the manufacturing process, both from economic and electrode strength standpoints. 
The life of the nickel-zinc system is often limited by the two electrochemical phenomena occuring on the zinc electrode - dendrite formation and shape change. Of these two problems, that associated with dendrites is probably the least serious in the present state of the technology and the more controllable one. A solution to the dendrite growth problem on recharge lies with the use of appropriate separation to prevent cell shorting. As discussed in Section II. B.1., Separator Materials, the probability of developing, in the next few years, a separator capable of at least 500 cycles in large cells is relatively high. By the same token, the development by Gould of a large zinc electrode capable of delivering that many cycles to about $70 \%$ of initial capacity in a large battery should, in our opinion, be available within the time period required to demonstrate the separator. It should be pointed out that, with the present state of technology on the zinc electrode, large nickel-zinc systems should be capable of 500 cycles to about $50 \%$ of original capacity, provided a separator with the same life expectancy was available. The following discussion is presented to substantiate the above statements.

The performance of full size electric vehicle electrodes is shown in Figures II-13 and II-14 for varying zinc masses. In these tri-electrode cell units, the capacity was limited by either the separator or the zinc electrode. As can be seen, a minimum of 500 cycles to about $50 \%$ of original capacity can be attained on large size electrodes. In these cells the capacity degradation was caused by shape change in the zinc electrode. Approximately 300 cycles to $80 \%$ of capacity was achieved with a later zinc electrode version before cell failure occurred due to separator degradation. The basic membrane separator in all cells was Celgard.

The data presented above show not only the progress made over a two year period, but gives an indication that a cycle life of about 500 cycles for large zinc electrodes (approximately one half a square foot) may be close at hand. Such electrodes, when used in the full size 400 Ah hardware, have been demonstrated to yield a cell with an energy density of 30 to $35 \mathrm{Wh} / \mathrm{lb}$ at the 6 hour rate. Since it is a known fact that the cycle life increases with increasing negative to positive capacity ratios (e.g., decreasing energy densities), it is highly probable that, at the expense of energy density, the large size cell could be built to achieve 500 useful cycles with present zinc electrode state of technology. Also, in much smaller size cells with small electrodes, the attainment of 500 cycles would be made easier at the lower energy densities. Consequently, any claims of 500 or more cycles in smaller cells can be misleading in terms of extropolation of the data to the large size cell whose energy density goal is greater than $30 \mathrm{Wh} / \mathrm{lb}$.

Shape change as it exists today can be attributed in part to some of the problems associated with the manufacture of large zinc electrode. As improved techniques are developed, a reduction in shape change and an increase in system life ( $>500$ cycles) are highly probable. This is not to say, however, that the solutions to the problems are simple. The need for practical and economical electrodes complicate the picture. 


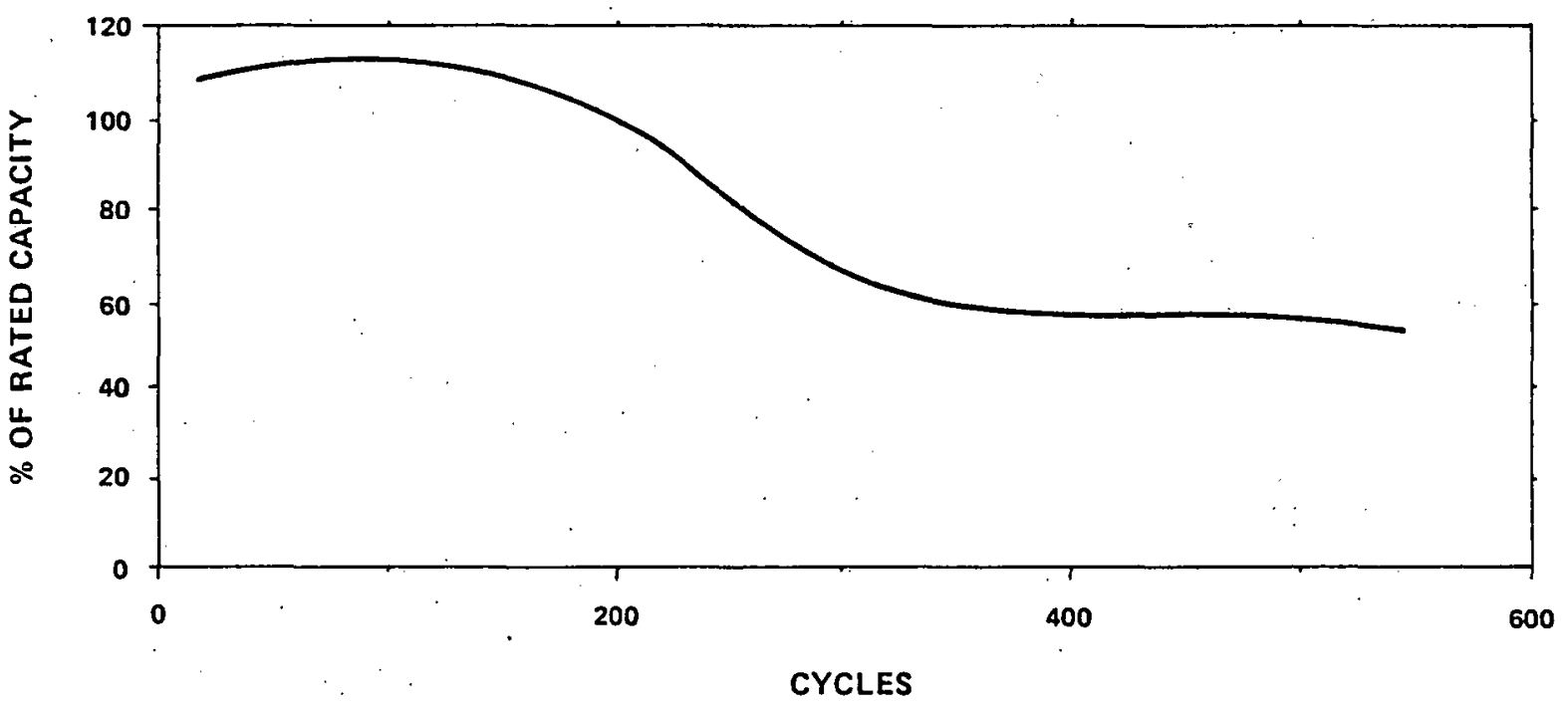

FIGURE 11-13:

50 Ah CELLS (400 Ah CELL COMPONENTS). 


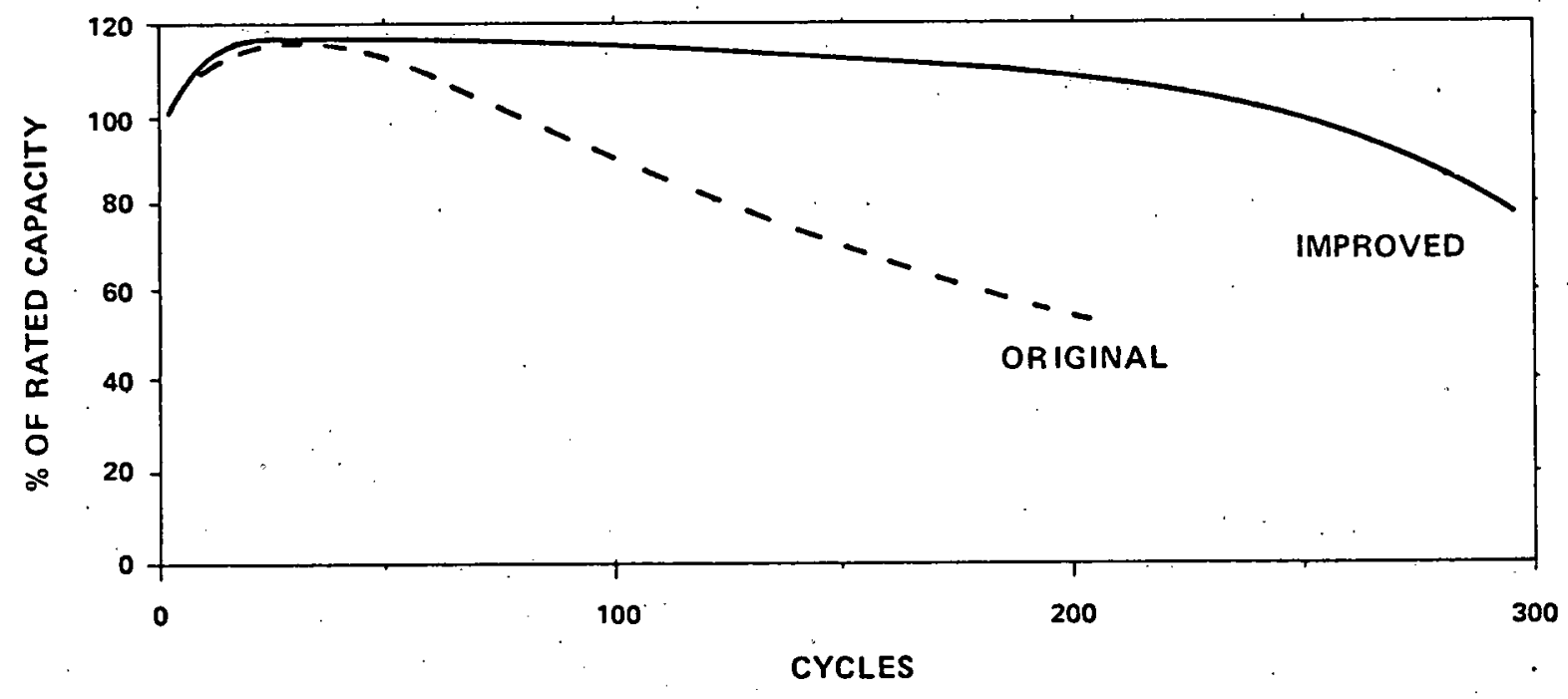

FIGURE II-14:

50 Ah CELLS (400 Ah CELL COMPONENTS) WITH ORIGINAL AND IMPROVED ZINC ELECTRODES. 


\section{Nickel Electrode}

Of the three key components, e.g., the separator, zinc electrode, and nickel electrode, the latter does not appear to be the life-limiting one in our nickel-zinc system. This has been primarily due to the fact that Gould employs a sintered rather than a non-sintered electrode. Gould's experience with both types goes back to the late 1950's. The past knowledge of the performance and manufacturing of these two basic electrodes has had much influence on our decision of recent years to emphasize our effort on a sintered nickel electrode. As a result, a reasonably low cost electrode was developed that, on an equivalent basis, is superior in performance to the non-sintered type. Furthermore, non-sintered electrodes equal in performance to the sintered type in every respect are not necessarily low cost.

While neither electrode types are free of problems, those associated with the sintered type are far less severe and generally pose little problems in the cell. On the other hand, the problems with the non-sintered electrode are numerous in a battery. This is particularly true when graphite instead of nickel powder is used as the conductive material. This has been known to Gould for quite some time (e.g., capacity loss presumably due to graphite oxidation in nickel-cadmium cells had been the subject of an extensive investigation by Gould researchers in the late fifties and into the sixties). Aside from this phenomenon, another well known difficulty is the severe swelling of the mass which occurs during cycling, resulting in loss of conductivity. In a recent publication (Ref. 3 ) by other workers engaged in the development of non-sintered electrodes for the nickel-zinc battery, the above two problems were identified as still existent.

Another problem connected with the non-sintered electrode which must be carefully analyzed is its inferior performance in the battery, particularly at higher discharge rates at end of discharge and at end of life. This situation becomes more prominent as the capacity per unit area of electrode increases (e.g., thicker electrodes) - a condition important in minimizing battery costs. Thicker electrodes and, consequently, smaller electrode area are permissible with the sintered type because of its higher efficiency at higher rates of discharge. This reduction in area makes possible the fabrication of a battery with a similarly smaller zinc electrode area in addition to less separation, all of which translate to lower material costs (e.g., positive and negative grids and separators) and lower labor costs in the manufacture of both electrodes and in the assembly of batteries. Furthermore, because of the more pronounced reduction in performance of the non-sintered type with increasing thickness, the total amount of mass significantly increases in relation to the sintered type if equivalent characteristics are to be achieved in the electric vehicle application.

Still another problem of concern with the performance of the nickel electrode, in particular the non-sintered type, is the cycle life in large batteries under the operating conditions of the electric vehicle. These conditions are more severe on the electrode and can accelerate their 
degradation. In view of its overall poorer performance, the non-sintered type would again be expected to have a life expectancy less than the sintered electrode, especially in a configuration that would be economical.

With respect to its manufacture, the non-sintered electrode may appear to involve simple and economical processes. However, many critical factors are often overlooked in analyzing the manufacturing of such electrodes. Among those is the preparation of efficient and economical active masses. Not only are commercial nickel hydroxide materials (one of the active mass ingredients) expensive, but they have been found in past studies at Gould to yield different performance results in an electrode, and were generally not comparable to materials produced by processes developed at Gould. Such processes can be complex and, consequently, can impose unreasonably high labor costs. Furthermore, the contribution of waste, scrap, and handling losses can turn out to be of major consequence.

Still another area that can pose greater problems with the non-sintered electrode is the probable higher scrap loss at the assembly line. Very minimal losses would be expected for the sintered electrode because of its high strength and ease of handling during assembly.

In the assessment of the problems with the nickel electrodes, it is thus essential to review them from the point of view of total system. Analyzing the differences between the two types, taking into consideration the costs, the manufacturing processes, and the total system parameters, can lead to the proper conclusion of the value of the sintered electrode over the non-sintered type.

It is our opinion that the Gould thick sintered electrode is cost competitive and superior to non-sintered types in the context of a total system. The data which was presented in Section I illustrated the good performance attained on our thick sintered electrode in large 400 Ah cells as well as in $40 \mathrm{Ah}, 12$ volt batteries. The cost analyses given in Section III reflect the type of electrodes used in the above cells and batteries, and also make projections based on other demonstrated data. 


\section{MANUFACTURING PROJECTIONS}

\section{A. Projected Manufacturing Processes \\ 1. Electrode Manufacturing Processes}

A key element of our nickel-zinc system is the use of relatively thick sintered nickel electrodes. Relative to conventional electrodes made from thin (e.g., 20 to $30 \mathrm{mil}$ ) sintered substrates, as used in sealed sintered-plate nickel-cadmium cells, the thick electrodes can be made with high capacity . per unit weight, and, more importantly, cost much less per unit of capacity. Values of 0.12 to 0.15 $\mathrm{Ah} / \mathrm{g}$ at up to $300 \mathrm{~A}$ (1.5 hour rate) have been demonstrated in actual cells ( $400 \mathrm{Ah}$ electric vehicle size). The energy density of the nickel electrode in the cell data shown in Table III-1 was $0.15 \mathrm{Ah} / \mathrm{g}$, based on actual cell measurements at $80 \mathrm{~A}$.

The development of our thick sintered nickel electrode for the nickel-zinc system has been based on the optimization of existing technology within Gould and the recognition of the opportunity which this presents. A great deal of the new technology was derived from experience in the development and manufacture of the thin sintered-plate electrodes for the nickel-cadmium cells going back to the late 1950 's.

The basic processes in the manufacture of our nickel electrode are shown in Figure III-1 in the form of a flow chart. Two specific operations are involved - one with the manufacture of a porous sintered nickel substrate and the other with the impregnation of the nickel substrate with active mass (nickel hydroxide and additive) formed directly in the pores of the substrate from a nickel solution that can be readily prepared from raw materials by simple economical techniques. Furthermore, the physical properties of the substrate (pore size and distribution, porosity, nickel powder content, strength) can be controlled, and porous substrates tailored to a particular requirement can be produced.

The labor cost to mass produce such a substrate and impregnate it with active mass is low in relation to cost, as shown in the cost breakdown analysis (Section III. C.). In contrast, the manufacture of non-sintered electrodes involves many steps which must be closely controlled - adding substantially to the labor cost. This is necessitated by the known facts that the physical properties of the nickel hydroxide, conductive material (e.g., graphite or nickel), capacity stabilizer additive (e.g., cobalt or the likes), and the binder can significantly influence the electrode characteristics, and that at least two of these products (nickel hydroxide and additive) must be manufactured under special conditions to meet the specifications. The manufacture of nickel hydroxide, for instance, can be quite complex, and can involve many process steps, some of which are given below to illustrate this point:

- Preparation of nickel salt from raw materials (this step is common to both types of electrodes) 
TABLE $\| I-1$

\section{GENERATION I ELECTRIC VEHICLE Ni-Zn CELL}

DESIGN A

CELLULOSIC SEPARATION (CELLOPHANE \& VISKING)

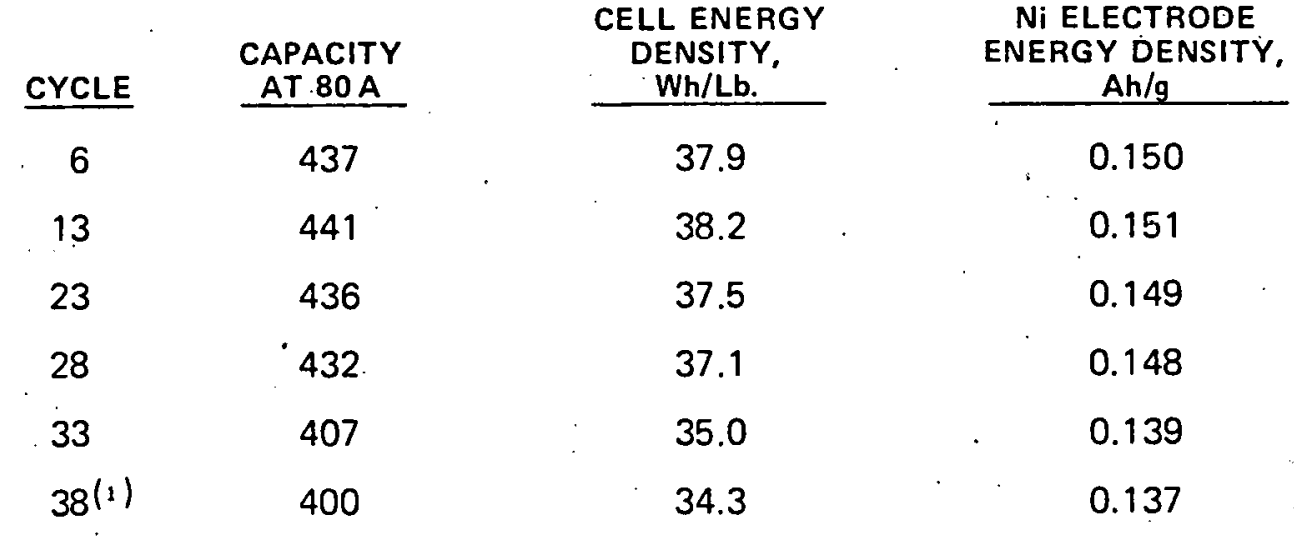

(1) Cycle life limited by degradation of cellulosic separators. 


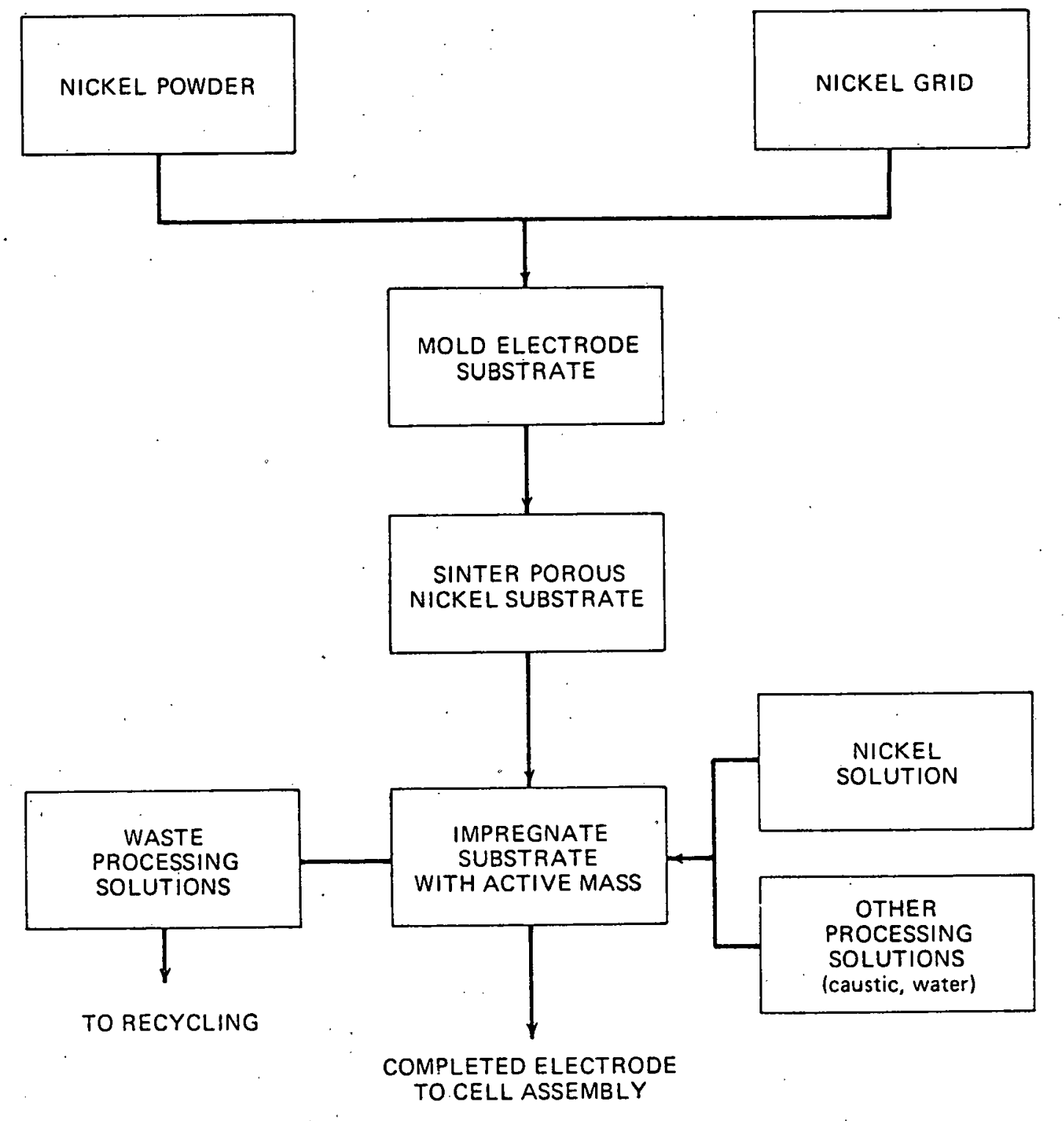

FIGURE III-1:

MANUFACTURE OF NIO ELECTRODE FOR ELECTRIC VEHICLE NICKELZINC BATTERY.

A. Basic Manufacturing Process. 
- Preparation of the nickel solution at the desired density, acidity, and temperature

- Precipitation of $\mathrm{Ni}(\mathrm{OH})_{2}$ (e.g., reacting the nickel solution with caustic)

- Filtration of the precipitated $\mathrm{Ni}(\mathrm{OH})_{2}$

- Washing free of caustic solution

- Drying of the $\mathrm{Ni}(\mathrm{OH})_{2}$

- Grinding of the $\mathrm{Ni}(\mathrm{OH})_{2}$

- Sizing of the $\mathrm{Ni}(\mathrm{OH})_{2}$

Although it is possible to co-precipitate the capacity stabilizer additive at the same time as the $\mathrm{Ni}(\mathrm{OH})_{2}$, it is uncertain that the best product would be produced. Consequently, it may be required to produce the additive separately by similar steps as described above. In either case; additional steps over and above those for the manufacture of these two ingredients are required and may include the following:

- Blending of $\mathrm{Ni}(\mathrm{OH})_{2}$, additive, and binder in precise amounts

- Possible pelletizing, grinding, and sizing

The production of finished electrodes is done by compacting this active material mixture onto a grid. This may be accomplished by rolling or pressing techniques in one or more steps, depending on a variety of parameters (e.g., grid and electrode designs).

Additional details on the manufacture of the nickel electrode are outlined in the flow charts in Figure III-2, Manufacture of Nickel Grid, Figure III-3, Manufacture of Nickel Solution, and Figure III-4, Recycling of Waste Processing Solutions. These processes are applicable to both electrode types.

The manufacture of the nickel solution has been done by Gould for quite some time in its nickel-cadmium battery plant and has been economically successful. The only deviation from the present operation is that the nickel from used nickel-zinc batteries would eventually become a significant factor in the source of nickel for the manufacture of the solution, at least after the population of the batteries in use has grown substantially.

The processes in the manufacture of our zinc electrode of the pressed type are shown in the flow diagram in Figure III-5. There are two basic operations, namely, the preparation of the zinc mass and the compaction of this mass onto a grid. The specific details in the preparation of the zinc mass may vary somewhat, depending on the form and proportion of the materials used. The 


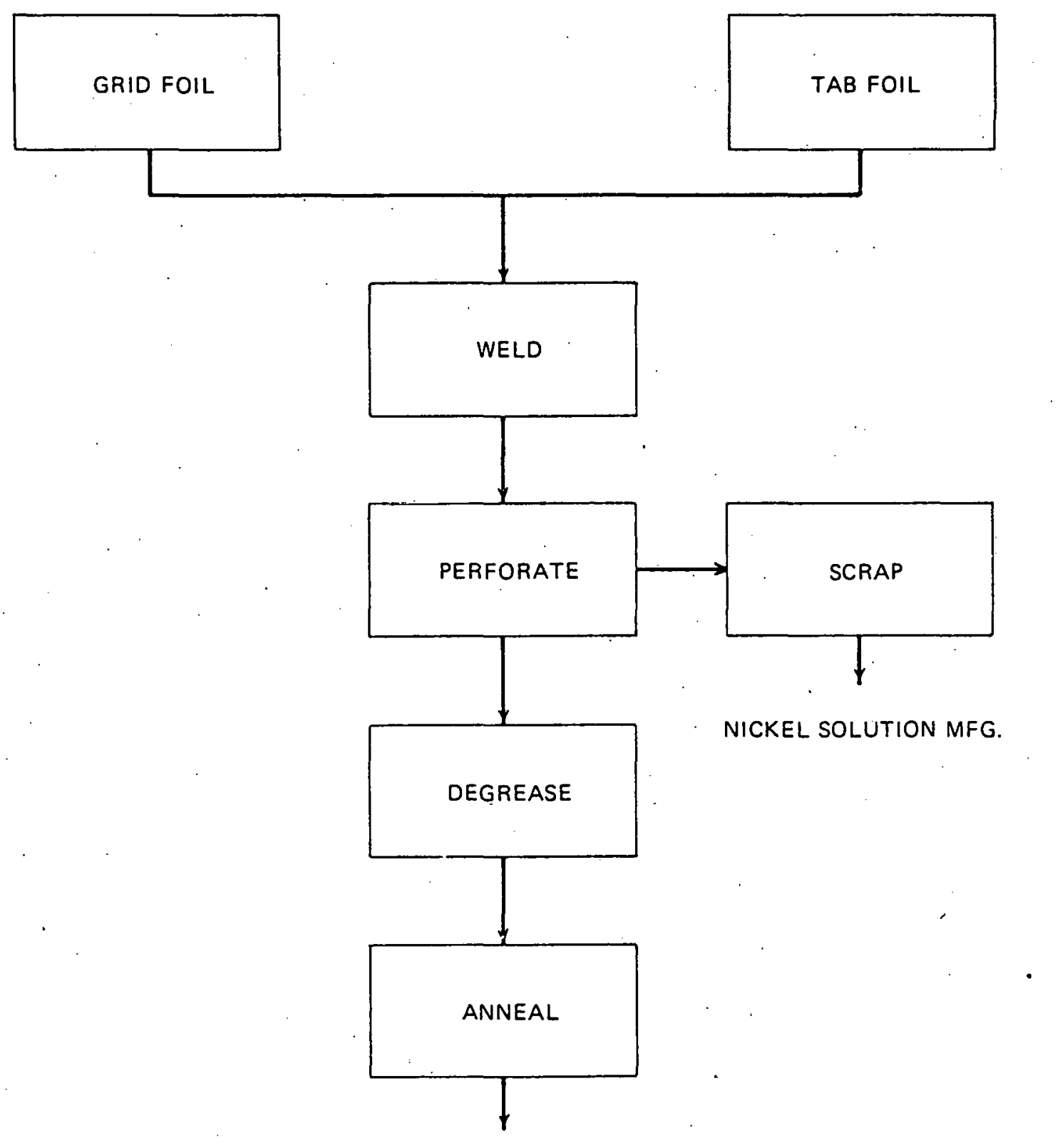

TO ELECTRODE MFG.

FIGURE III.2:

MANUFACTURE OF NIO ELECTRODE FOR ELECTRIC VEHICLE NICKEL. ZINC BATTERY.

B. Manufacture of Nickel Grid. 


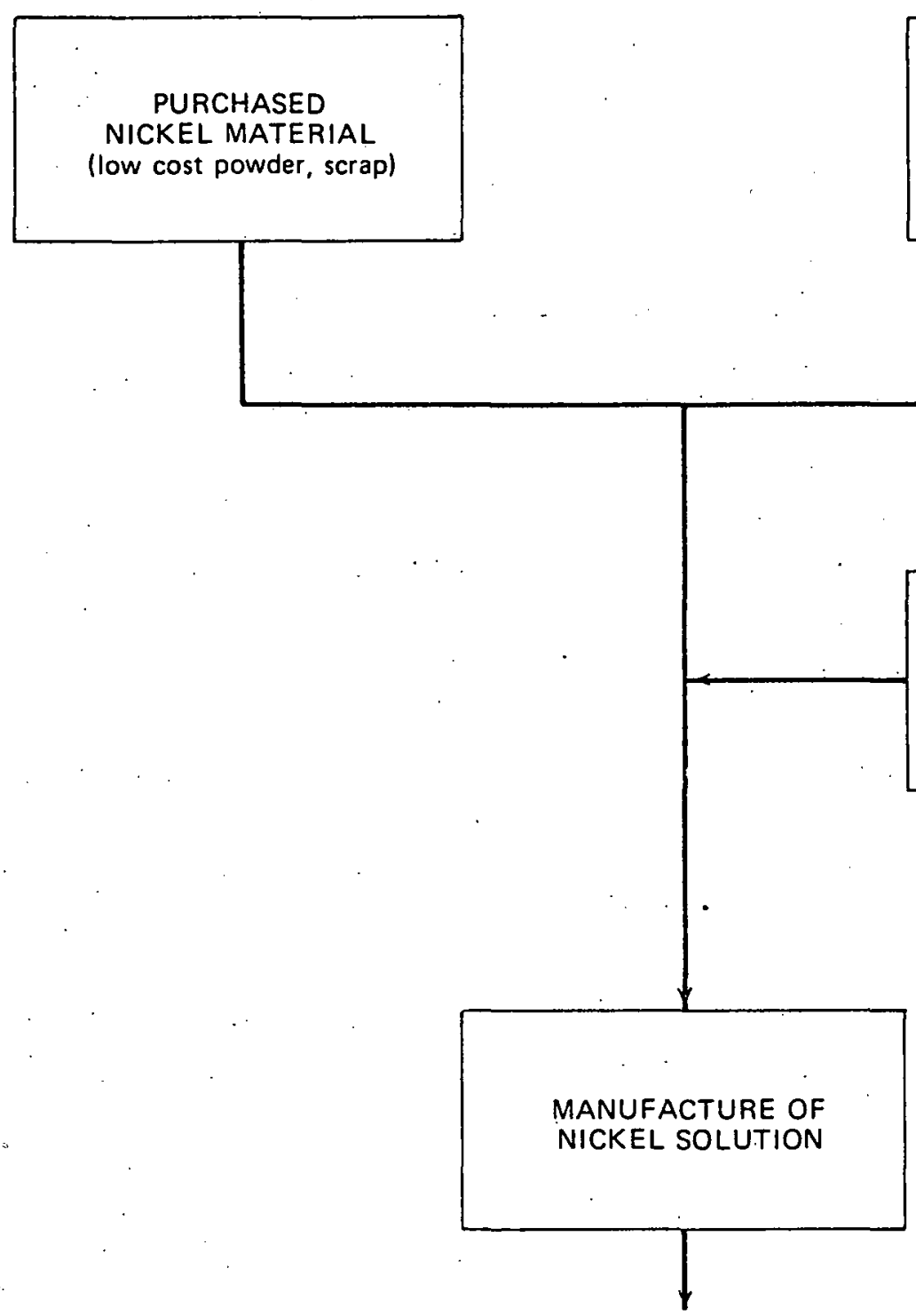

SCRAP MATERIAL FROM GRID MFG., i.e., PERFORATIONS

OTHER PROCESS SCRAPS:

- GRIDS (POS. \& NEG.)

- NICKEL POWDER

- SINTERED SUBSTRATES

- NIO ELECTRODES

TO IMPREGNATION PROCESS

FIGURE III-3:

MANUFACTURE OF NIO ELECTRODE FOR ELECTRIC VEHICLE NICKEL. ZINC BATTERY.

C. Manufacture of Nickel Solution. 


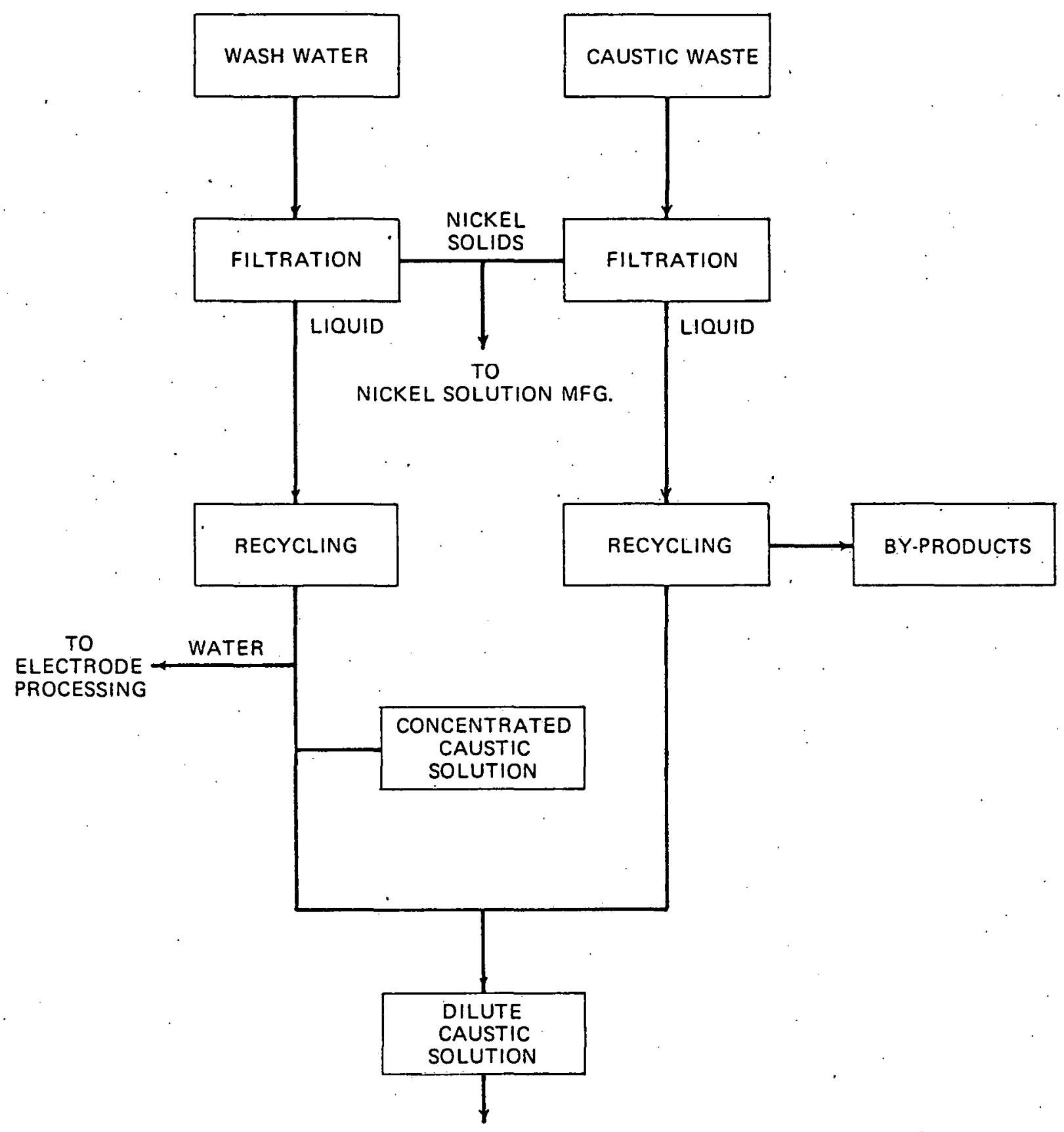

TO ELECTRODE PROCESSING

FIGURE III-4:

MANUFACTURE OF NIO ELECTRODE FOR ELECTRIC VEHICLE NICKEL. ZINC BATTERY.

D. Recycling of Waste Processing Solutions. 


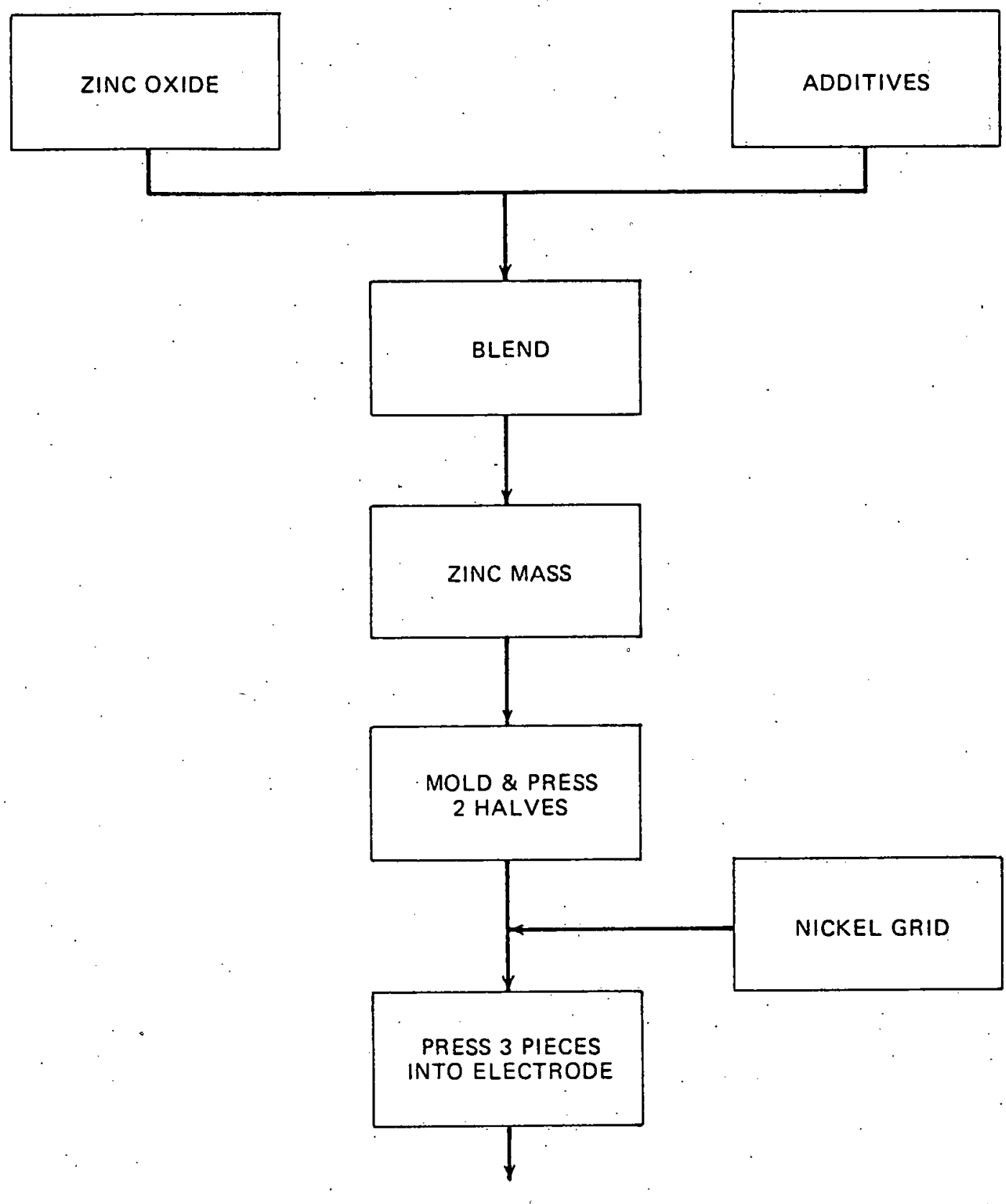

TO CELL ASSEMBLY

FIGURE 111-5:

MANUFACTURE OF ZINC ELECTRODE FOR ELECTRIC VEHICLE NICKEL-ZINC BATTERY.

A. Basic Manufacturing Processes. 
steps in the manufacture of the grid (Figure III-6) are essentially identical to those for the nickel electrode, with the exception, perhaps, in the design of the grid. The compaction of the zinc mass can be accomplished by a variety of approaches. The specific manufacturing techniques used depend largely on the electrode configuration desired. Generally, the compaction can be done on conventional equipment. In volume production, the operations of molding and pressing are envisioned at the present as being done by conventional high speed presses adapted for this use. Electrodes in the final configuration would be produced. The single electrode operation offers greater electrode design flexibility. These compaction steps would be automated to the point necessary for high volume and low cost production.

\section{Separator Manufacturing Processes}

As indicated earlier in this report, Gould has been developing new separators for the nickelzinc battery, including inorganic/organic composite types, which hopefully will be the material of first choice. The key steps in the manufacture of this material are given in Figure III-7. Specific details are withheld because of the possible proprietary aspect of certain processes in the manufacturing of these materials. As can be seen, however, the process involves relatively few simple operations, although certain steps (e.g., those in the materials formulation and handling) require that they be carried out precisely to achieve a quality product with the desired physical properties. The important point is that the application demands a uniform pore size separator to assure iong cycle life. The less complex the process, the more reliable the product should be in mass production.

\section{Manufacturing Assembly Processes}

The assembly of the nickel-zinc cell, in our opinion, poses no greater obstacles than other volume production batteries such as the automotive lead-acid type which can be manufactured at low cost. Highly automated assembly lines are possible for the nickel-zinc product. However, for both economical and technical reasons, a proper balance in the degree of automation will be necessary in the early years of production. Past battery manufacturing experience at Gould dictates this course of action. Only through volume production experience will highly automated assembly processes be developed that will be technically successful. Also, due to the nature of any new product, changes in the product are likely in the early years of production that could require modifications in the assembly techniques or new processes.

The selected electrode configurations, separator system, cell design, and hardware design will influence the assembly processes. While certain assembly processes cannot be well defined at present or are in the conceptual stage, it can be stated that many process approaches are derived to some extent from our knowledge of the manufacture of batteries in large volume (e.g., automotive and industrial lead-acid batteries) and to some degree from our manufacturing operations in the nickel-cadmium and silver-zinc cells. 


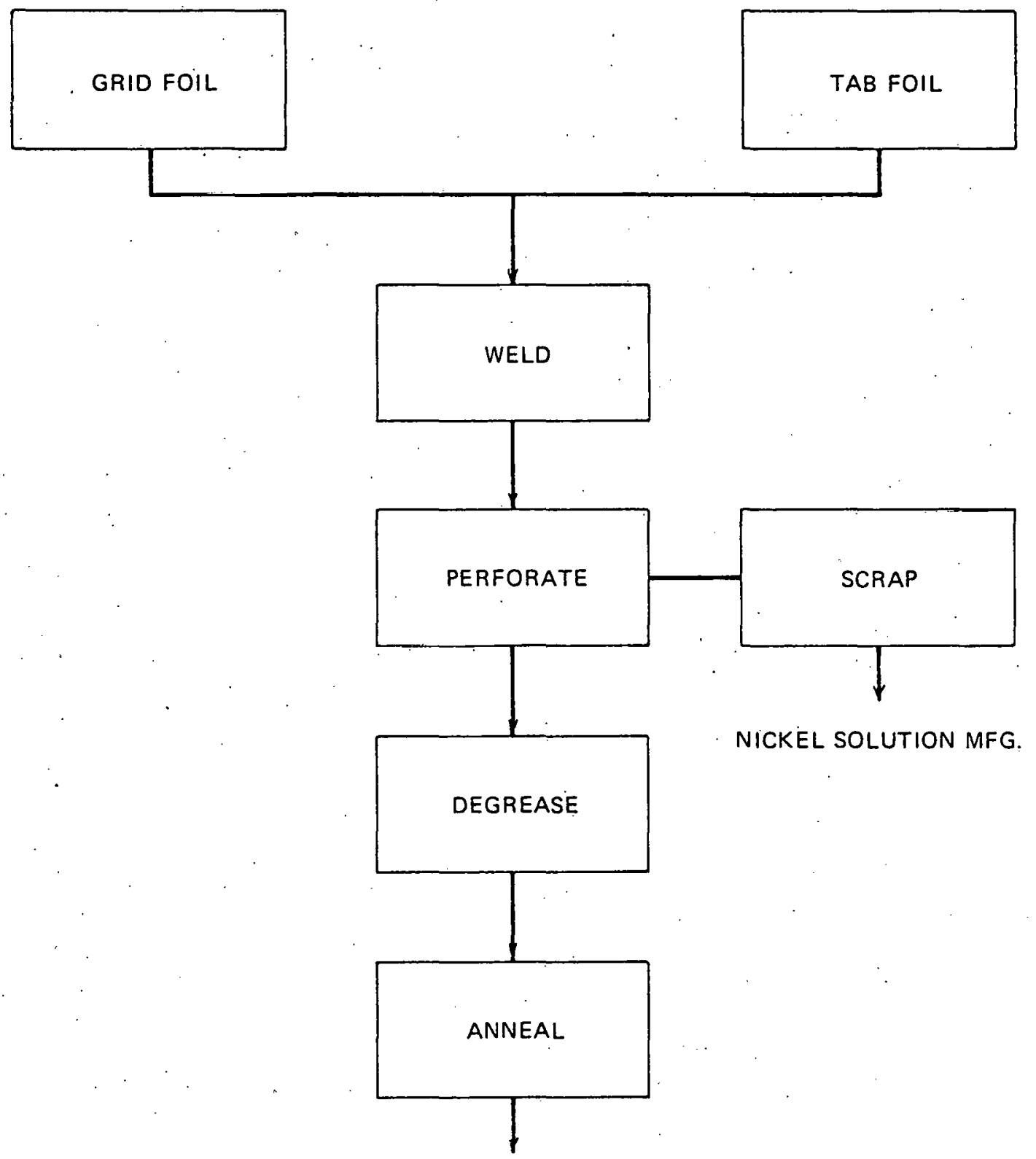

TO ÉLECTRODE MFG.

FIGURE III-6:

MANUFACTURE OF ZINC ELECTRODE FOR ELECTRIC VEHICLE NICKEL-ZINC BATTERY.

B. Manufacture of Nickel Grid. 


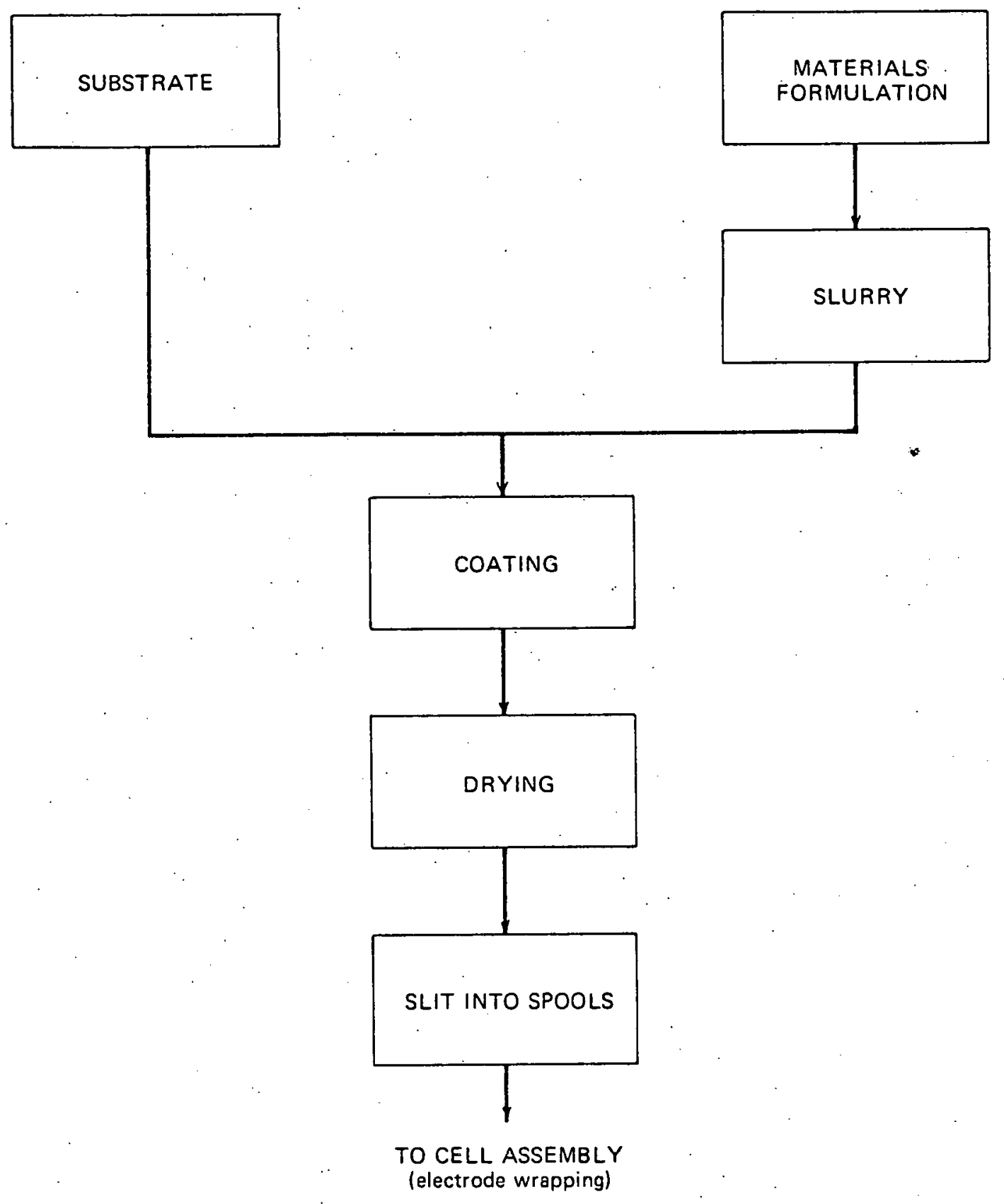

FIGURE 1II-7:

MANUFACTURE OF FLEXISLE INORGANIC/ORGANIC COMPOSITE SEPARATOR. 
The key process in the assembly of the cell as we see it now will be that connected with the separator system, e.g., the steps in the wrapping of electrodes with the separators. Again, the selected separator materials may dictate the process approach. With the development of flexible separators, we envision that this operation, the critical one in the assembly, will be automated for economical reasons. Such machines have been demonstrated recently in high rate production of lead-acid batteries where a flexible separator is used in place of the more rigid conventional separator. In this equipment, about 30 electrodes per minute are wrapped with the separator.

In our opinion, assembly processes are not going to be critical factors in determining the manufacturing economics. Also, for the reasons previously stated, the various assembly processes cannot be specifically detailed at this stage in the development, nor can some be divulged because of their probable proprietary nature. Consequently, only the general processes are outlined in Figure III-8.

\section{Processes in Recycling of Used Batteries}

Gould believes that recycling of the materials in the battery will have considerable potential impact on the cost of manufacturing the nickel-zinc battery. A major process would involve the recovery of the nickel values to produce the nickel solution used in the manufacture of the nickel electrode. The manufacture of the nickel solution has been discussed previously (Section III. A.1.). An alternative, which may also be possible, is the recycling of electrodes whole after a simple cleaning operation. This might be made possible because of the sintered type electrode. The economics of recycling the electrodes whole versus the production of the nickel solution must be weighed carefully, however, in view of the higher initial cost of a longer cycle life electrode and its impact on the cost increase of the battery. These two recycling processes are shown in Figures III-9 and III-10.

Appropriate processing schemes could presumably also be worked out for the separate recovery of the zinc mass. Likewise, it is conceivable that the plastic materials (case and cover) could be recycled. Metallic components other than the nickel grids and tabs, namely, terminals, cell and battery connectors, and battery module packaging hardware could be salvaged.

\section{B. Battery Manufacturing Cost Estimates}

The projected manufacturing cost estimates for the electric vehicle cells show materials plus labor at $\$ 33.91 / \mathrm{kWh}$, based on a production volume of $326,000 \mathrm{kWh}$ per year (equivalent to $13,040-25 \mathrm{kWh}$ batteries or $400,000-500 \mathrm{Ah}$ cells). The cost is somewhat sensitive to the cell design as well as capacity size. For instance, a cell with a capacity about half of that given above (approximately $250 \mathrm{Ah}$ ) would be about $12 \%$ higher in cost.

The manufacturing costs for the nickel-zinc cells are particularly sensitive to the prices assumed for certain materials (e.g., nickel and nickel products, zinc oxide, additives, and binders) and to a lesser degree to the hourly labor rate and the overhead factor. The costs are also based on the 

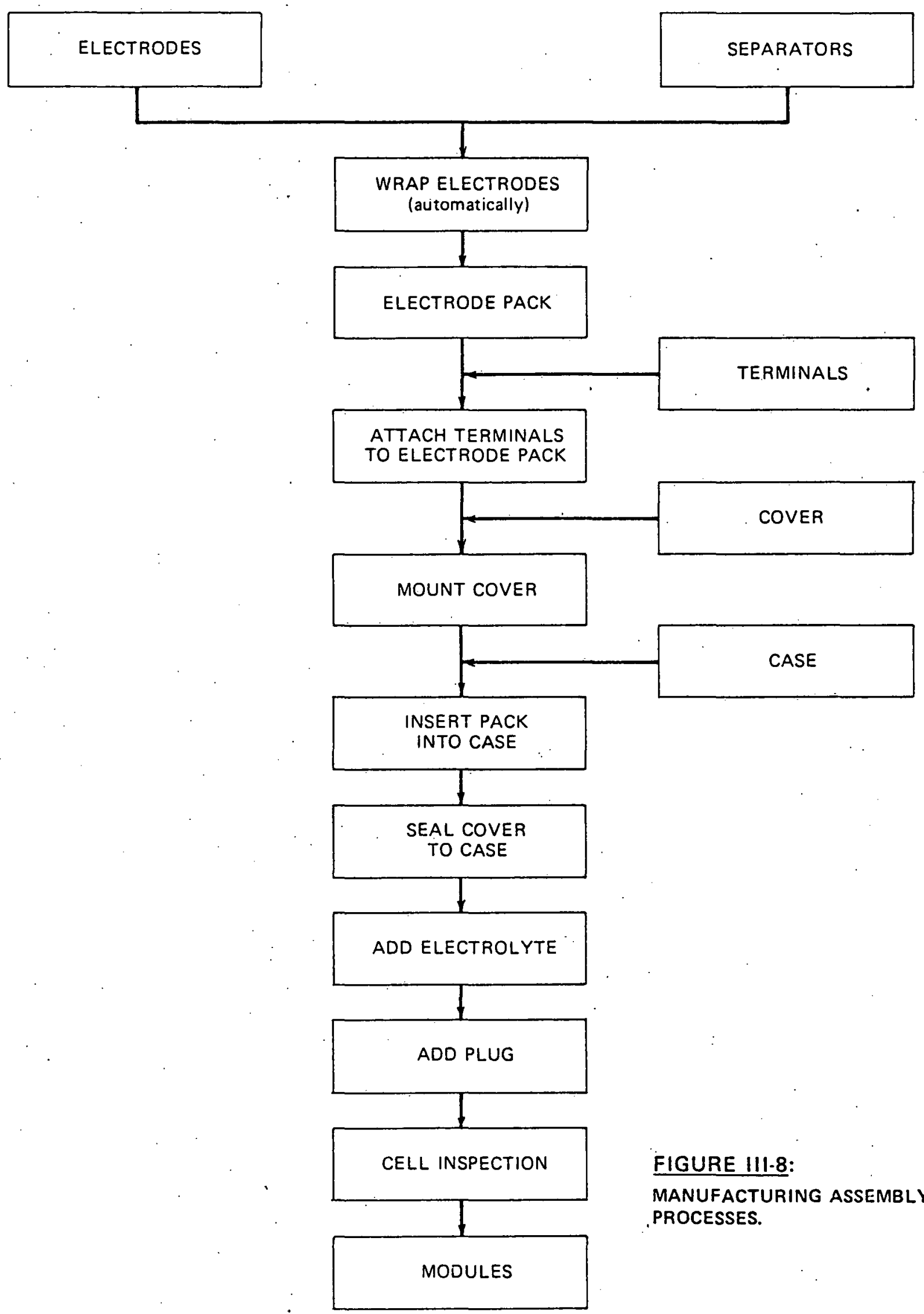

FIGURE 111-8:

MANUFACTURING ASSEMBLY PROCESSES. 


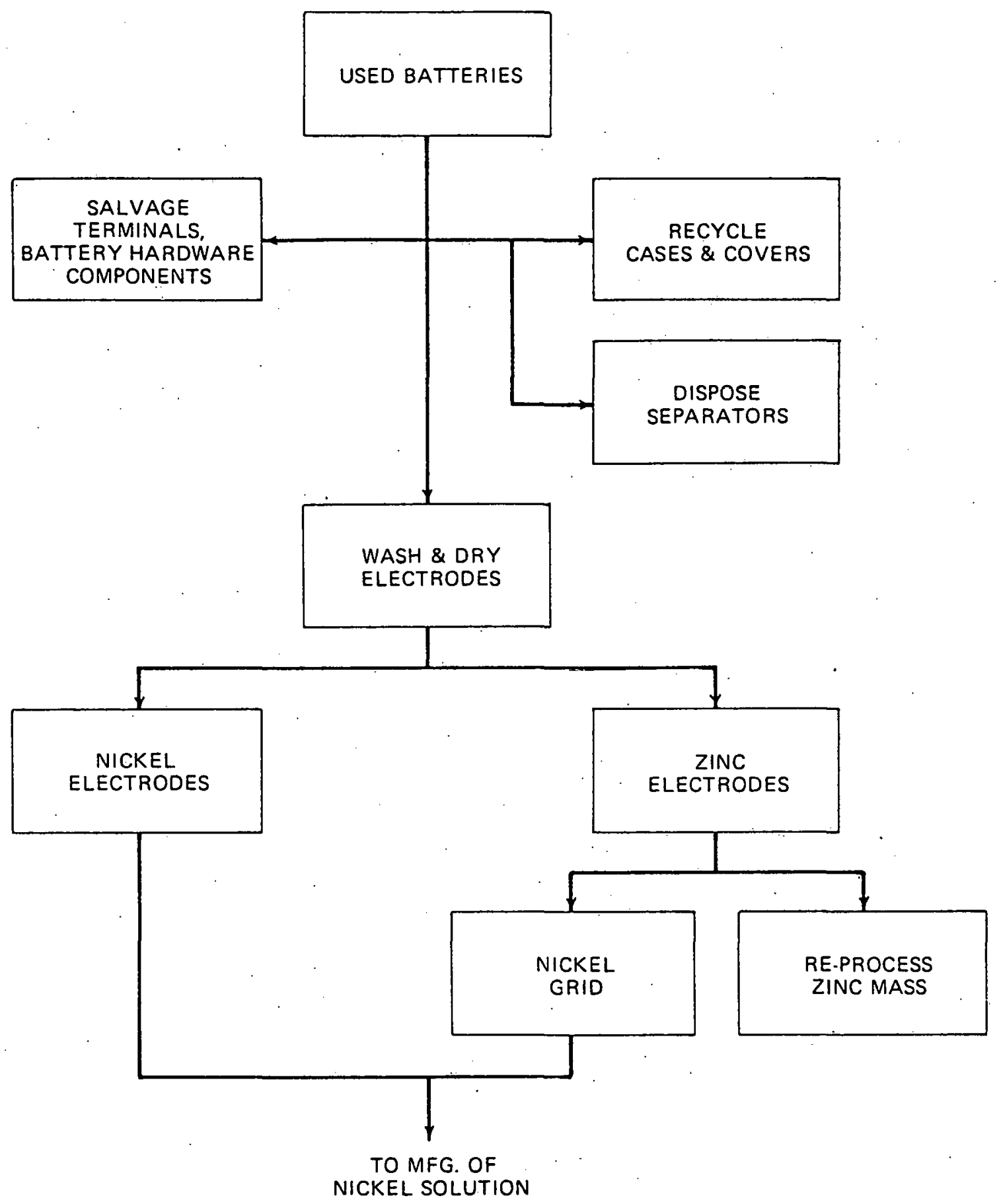

FIGURE III-9:

RECYCLING OF USED NICKEL-ZINC BATTERIES.

A. Presently Proposed Process. 


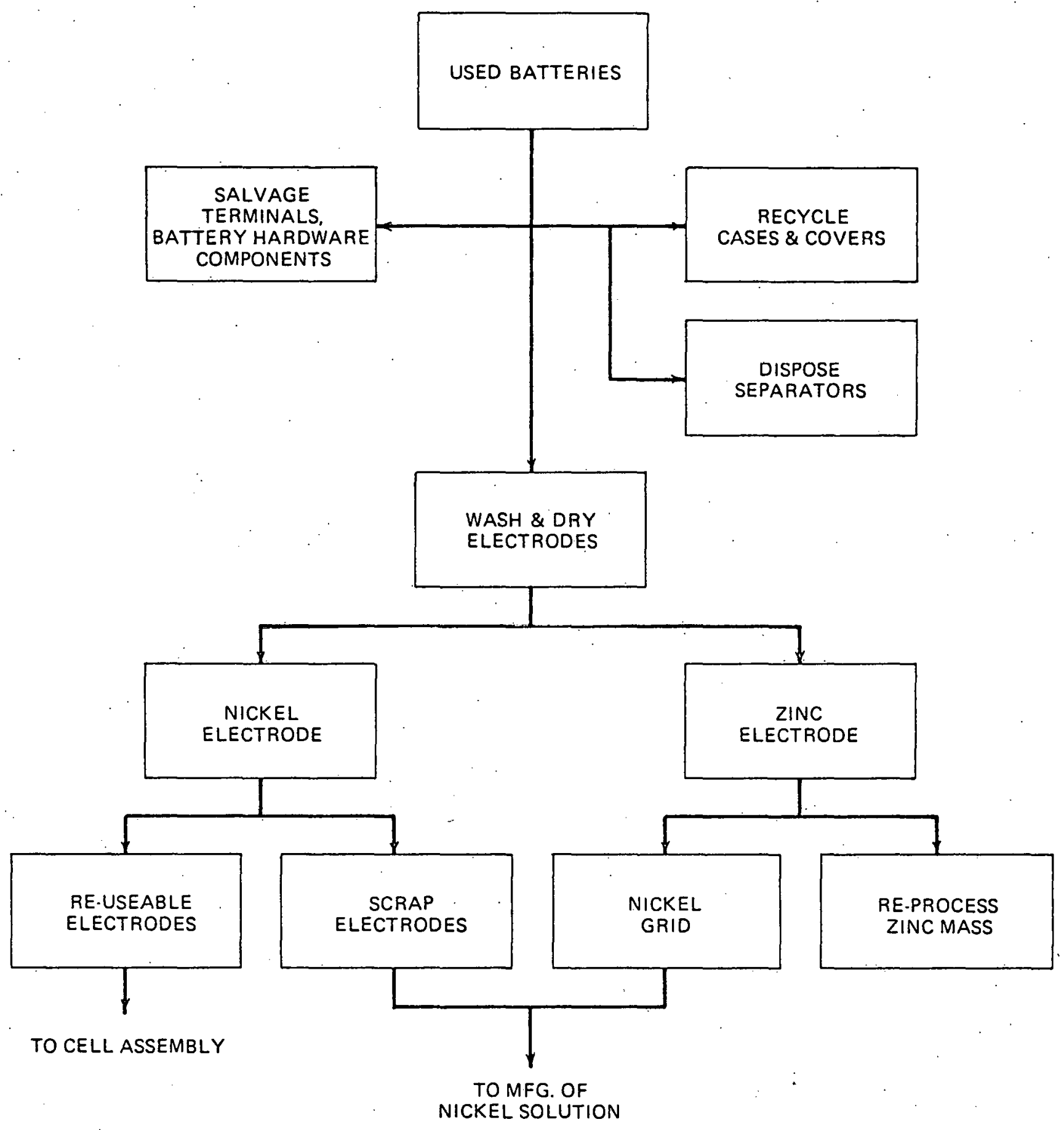

FIGURE III-10:

RECYCLING OF USED NICKEL-ZINC BATTERIES.

B. Alternate Process Approach. 
assumption that a suitable low cost separator is developed. Failure to accomplish this would result in a possible substantial increase in manufacturing costs.

The prices used for the different purchased materials were taken from quotations in August and September of 1976 . The above cost estimates make no allowance for the potential savings which would accrue from the recycling of the batteries, and either lower than $100 \%$ capacity utilization or higher than normal labor needed in the early phases of production.

The projected manufacturing cost estimates at the pilot plant level (e.g., 1,304 - $25 \mathrm{kWh}$ batteries per year) can vary depending on the capital investment made. For a reasonably advanced operation, the cost would be around $\$ 58.72 / \mathrm{kWh}$. For a 6,520 battery plant, a cost of $\$ 45.71 / \mathrm{kWh}$ is estimated.

Production process costs are summarized in Tables III- 2 for pilot (1,304 and 6,520 batteries) and production (13,040 and 130,400 batteries) levels, Tables III-3 through III-5 summarize the costs by components. A detailed cost breakdown is given in Tables III-6a through III-6d for the production level of 13,040 batteries per year. A breakdown for the larger production facility (i.e., 130,400 batteries) is not included since the only difference in cost is in a very slight reduction in the conversion rates (equipment and labor). However, this does not imply that further cost reductions are not possible. For instance, the larger material volumes may make it possible to negotiate lower prices with the raw material suppliers.

\section{Capital Investment Estimates}

The capital requirements for pilot facilities capable of producing 1,304 and $6,520-25 \mathrm{kWh}$ batteries per year, and for production facilities capable of producing 13,040 and 130,400-25 kWh batteries per year, are summarized in Table III-7. The values assume the cost for plant buildings (including the cost for adapting the plants to our needs), and for process equipment. They do not include the requirements for special test equipment associated with manufacturing of batteries and quality control provisions.

A breakdown of the capital requirements, by major operations, is given in Table III-8 for pilot and production facilities.

\section{Materials Requirements}

Requirements for the major materials are shown in Table III-9 for pilot and production facilities. The requirements for nickel, cobalt, zinc, copper, and polypropylene for a nickel-zinc production facility of $130,400-25 \mathrm{kWh}$ batteries per year are compared in Table III-10 against 1975 world productions. The requirements for battery production of this size would have no significant impact on the supply of materials. Nickel shipments through 1985 are projected to continue growing at $6.5 \%$ per year.

\section{E. Pilot Production Facility Layout}

A sketch/floor plan of Gould's Nickel-Zinc Battery Process Demonstration Facility (Pilot Plant) is shown in Figure III-11. The installation of this facility is expected to be completed during calendar year 1977. 
TABLE $\quad \| I-2$

SUMMARY OF MANUFACTURING COST ESTIMATES ${ }^{(1)}$

ELECTRIC VEHICLE Ni-Zn CELL

500 Ah (815 Wh) AT 3 to 6 HOUR RATE

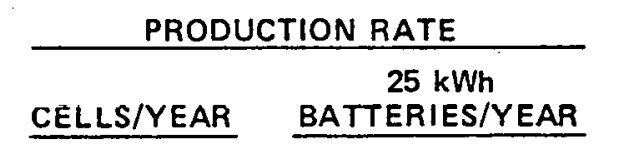

\section{PILOT}

40,000

200,000

PRODUCTION

400,000

$4,000,000$
13,040

130,400
1,304

6,520

6,520

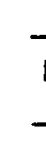

\begin{tabular}{|c|c|c|c|}
\hline $\begin{array}{c}\text { MATERIALS } \\
\$\end{array}$ & $\begin{array}{c}\text { CONVERSION }^{(2)} \\
\$\end{array}$ & $\begin{array}{c}\text { TOTAL } \\
\$ \\
\end{array}$ & $\begin{array}{c}\cos T / k W h \\
\$\end{array}$ \\
\hline 26.72 & 21.14 & 47.86 & 58.72 \\
\hline 26.18 & 11.08 & 37.26 & 45.71 \\
\hline 21.64 & 6.24 & 27.88 & 34.20 \\
\hline $21.64^{(3)}$ & 6.00 & $27.64^{(3)}$ & $33.91^{(3)}$ \\
\hline
\end{tabular}

COST PER CELL

\begin{tabular}{|c|c|c|c|}
\hline \multicolumn{3}{|c|}{ COST PER CELL } & \multirow[b]{2}{*}{$\begin{array}{c}\cos T / k W h \\
\$\end{array}$} \\
\hline $\begin{array}{c}\text { MATERIALS } \\
\$ \\
\end{array}$ & $\begin{array}{c}\text { CONVERSION } \\
\$ \\
\end{array}$ & $\begin{array}{c}\text { TOTAL } \\
\$ \\
\end{array}$ & \\
\hline 26.72 & 21.14 & 47.86 & 58.72 \\
\hline 26.18 & 11.08 & 37.26 & 45.71 \\
\hline 21.64 & 6.24 & 27.88 & 34.20 \\
\hline $2.1 .64^{(3)}$ & 6.00 & $27.64^{(3)}$ & $33.91^{(3)}$ \\
\hline
\end{tabular}

(1) Costs Before Battery Recycling

(2) Costs For Equipment and Labor

(3) Does Not Reflect Possible Lower Material Costs in Higher Volumes Than 13,040 Batteries 
TABLE III-3

MANUFACTURING COST ESTIMATE

400,000 - 500 Ah CELLS PER YEAR

(13,040 - 25 kWh BATTERIES)

\begin{tabular}{|c|c|c|c|}
\hline . & $\begin{array}{c}\text { MATERIALS } \\
\$\end{array}$ & $\begin{array}{c}\text { CONVERSION } \\
S\end{array}$ & $\begin{array}{l}\text { TOTAL } \\
\$ \\
\end{array}$ \\
\hline NICKEL ELECTRODE & 14.38 & 3.27 & 17.65 \\
\hline ZINC ELECTRODE & 4.00 & 1.96 & 5.96 \\
\hline HARDWARE & 2.04 & --- & 2.04 \\
\hline SEPARATOR & 1.00 & 0.24 & 1.24 \\
\hline ELECTROLYTE & 0.22 & --- & 0.22 \\
\hline ASSEMBLY & $\div-$ & 0.77 & 0.77 \\
\hline \$/CELL: & 21.64 & 6.24 & 27.88 \\
\hline$\$ / \mathrm{kWh}:$ & 26.55 & 7.65 & 34.20 \\
\hline
\end{tabular}


TABLE $\| i \mid .4$

MANUFACTURING COST ESTIMATE

$$
\begin{gathered}
200,000-500 \text { Ah CELLS PER YEAR } \\
(6,520-25 \text { kWh BATTERIES })
\end{gathered}
$$

\begin{tabular}{|c|c|c|c|}
\hline & $\begin{array}{c}\text { MATERIALS } \\
\$ \\
\end{array}$ & $\begin{array}{c}\text { CONVERSION } \\
\mathrm{S}\end{array}$ & $\begin{array}{c}\text { TOTAL } \\
\$ \\
\end{array}$ \\
\hline NICKEL ELECTRODE & 16.39 & 7.03 & 23.42 \\
\hline ZINC ELECTRODE & 6.37 & 2.27 & 8.64 \\
\hline HARDWARE & 2.20 & $-\infty$ & 2.20 \\
\hline SEPARATOR & 1.00 & 0.24 & 1.24 \\
\hline ELECTROLYTE & 0.22 & $-\infty$ & 0.22 \\
\hline ASSEMBLY & -- & 1.54 & 1.54 \\
\hline \$/CELL: & 26.18 & 11.08 & 37.26 \\
\hline$\$ / \mathrm{kWh}:$ & 32.12 & 13.59 & 45.71 \\
\hline
\end{tabular}


TABLE III-5

MANUFACTURING COST ESTIMATE

$$
\begin{aligned}
& \text { 40,000 - } 500 \text { Ah CELLS PER YEAR } \\
& (1,304-25 \text { kWh BATTERIES })
\end{aligned}
$$

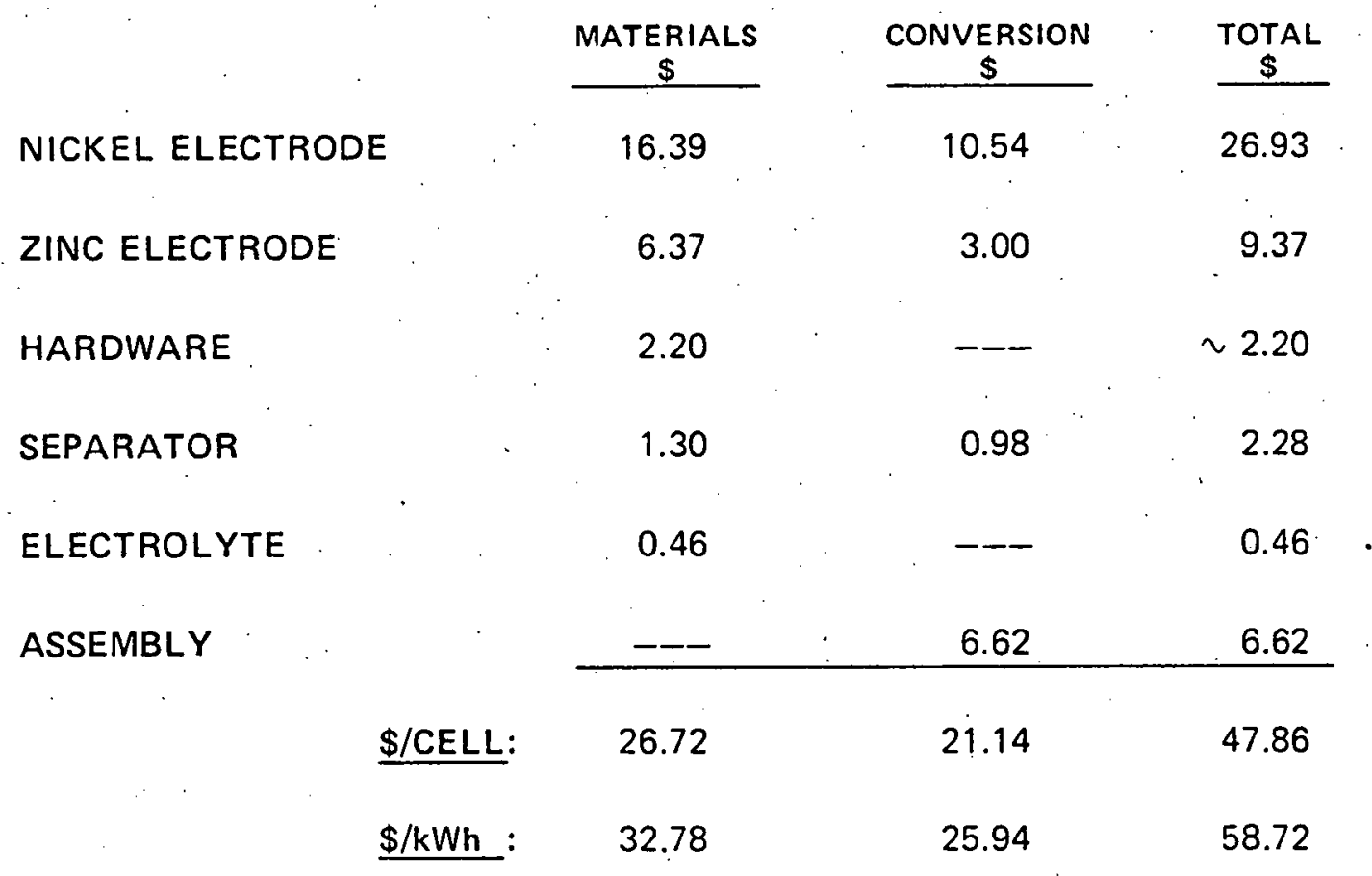


TABLE $111.6 a$

\section{DETAILED COST BREAKDOWN*}

13,040 - 25 kWh BATTERIES PER YEAR

(400,000 - 500 Ah CELLS)

MATERIALS REQUIREMENT - PER CELL

PROCESSES

NICKEL ELECTRODE MFG.

Ni SUBSTRATE FABRICATION

TAB PREPARATION
GRID PREPARATION
WELD TAB TO GRID
NI POWDER BLEND
MOLD SUBSTRATE
SINTER SUBSTRATE
COIN SUBSTRATE

IMPREGNATION

Ni SOLUTION

(Including Additive)

PROCESS SOLUTIONS

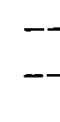

$\begin{array}{cc}0.1120 & 0.0186 \\ 0.1624 & 0.0033 \\ -- & -- \\ 3.0000 & 0.0456 \\ -- & --- \\ -- & -- \\ -- & ---\end{array}$

0.1306
0.1657
---
3.0456
---
---
---

4.90

9.00

$---$

2.55

$--$

$--$

mat

$\begin{array}{r}\text { TOTAL } \\ \text { MATERIA } \\ \hline\end{array}$

$\$ / 16$

MATER
CONVERSION - \$/CELL

EQUIP. LABOR $\begin{gathered}\text { TOTAL } \\ \text { CONVERSION }\end{gathered}$

\section{EQUIPMENT \& LABOR}

Eff., $80 \%$

\begin{tabular}{|c|c|c|c|c|c|c|}
\hline$--\dot{-}$ & 9.65 & 0.40 & $\begin{array}{l}3.86 \\
0.20\end{array}$ & 0.1435 & 0.3643 & 0.5078 \\
\hline & & & & 1.4031 & 1.2461 & 2.6492 \\
\hline--- & --- & -- & $\ldots$ & 0.2806 & 0.2492 & 0.5298 \\
\hline & & & $13.96^{\circ}$ & 1.6837 & 1.4953 & 3.1790 \\
\hline--- & - - & $-\therefore-$ & 0.42 & 0.0505 & 0.0448 & 0.0953 \\
\hline & --- & -- & 14.38 & 1.7342 & 1.5401 & 3.2743 \\
\hline
\end{tabular}

TOTAL COST:

* See Appendix A, Partial Listing of Material Suppliers and Equipment Manufacturers.

* Chosen arbitrarily at $3 \%$, actual will vary significantly depending on total Gould Ni-Zn battery business and the nature of industry which evolves. 


\section{TABLE $111-6 b$}

\section{DETAILED COST BREAKDOWN *}

13,040 - 25 kWh BATTERIES PER YEAR

(400,000 - 500 Ah CELLS)

\section{PROCESSES}

ZINC ELECTRODE MFG.

MATERIALS PREPARATION

TAB PREPARATION

GRID PREPARATION

WELD TAB TO GRID

MASS BLEND

$$
\mathrm{ZnO}
$$

BINDER

§

\section{MATERIALS REQUIREMENT - PER CELL}

\begin{tabular}{|c|c|c|c|c|}
\hline lb/CELL & $\underset{\mathbf{l b}}{\operatorname{SCRAP}}$ & $\underset{\mathbf{I b}}{\text { TOTAL }}$ & $\begin{array}{c}\text { PRICE } \\
\$ / / \mathbf{b}\end{array}$ & $\begin{array}{l}\text { TOTAL } \\
\text { MATERIALS }\end{array}$ \\
\hline
\end{tabular}

CONVERSION - \$/CELL

EQUIP. LABOR CONVERSION

\section{COMPACTION}

MOLD/PRESS HALVES PRESS ELECTRODE

$\begin{array}{lccccccc}0.1260 & 0.0209 & 0.1469 & 4.90 & 0.72 & 0.0173 & 0.0332 & 0.0505 \\ 0.1880 & 0.0039 & 0.1919 & 9.00 & 1.73 & 0.0393 & 0.0283 & 0.0676 \\ -- & --- & --- & --- & -- & 0.0185 & 0.0811 & 0.0996 \\ & & & & & & & \\ 2.8480 & 0.0432 & 2.8912 & 0.44 & 1.26 \\ 0.7120 & 0.0108 & 0.7228 & 0.25 & 0.18\end{array}$

EQUIPMENT \& LABOR Eff., $80 \%$

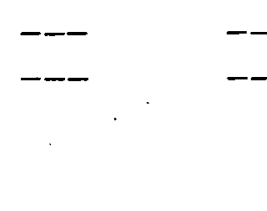

AS \& $E, 3 \%$ **

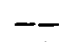

TOTAL COST:
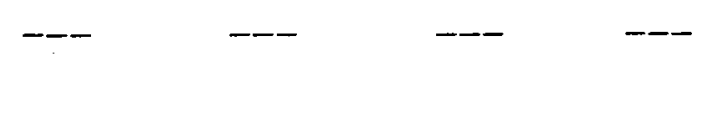

$\underline{0.11}$

$\underline{0.0116}$

$\underline{0.0454}$

$\underline{0.0570}$

4.00

0.3996

1.5610

1.9606

* See Appendix A, Partial Listing of Material Suppliers and Equipment Manufacturers.

* Chosen arbitrarily at $3 \%$, actual will vary significantly depending on total Gould Ni-Zn battery business and the nature of industry which evolves. 
TABLE III-6C DETAILED COST BREAKDOWN *

13,040 - $25 \mathrm{kWh}$ BATTERIES PER YEAR (400,000 - 500 Ah CELLS)

PROCESSES

㝏

\section{SEPARATORS MFG.}

I/O MIX

SUBSTRATE

EQUIPMENT \& LABOR Eff., $80 \%$

AS \& $E, 3 \%$ **

TOTAL COST:
MATERIALS REQUIREMENT - PER CELL

\begin{tabular}{|c|c|c|c|}
\hline |b/CELL & $\underset{\mathbf{I b}}{\text { SCRAP }}$ & $\underset{\text { Ib }}{\text { TOTAL }}$ & $\begin{array}{c}\text { PRICE } \\
\$ / I b\end{array}$ \\
\hline
\end{tabular}
TOTAL
ATERIALS S CONVERSION - \$/CELL EQUIP. LABOR $\begin{gathered}\text { TOTAL } \\ \text { CONVERSION }\end{gathered}$
0.950

1.062

0.118

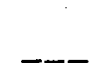

1.00

1.18

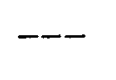

\subsection{8}

0.50

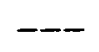

$\left.\begin{array}{l}0.38 \\ 0.59\end{array}\right\}$

0.0600

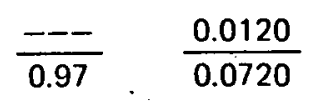

$\frac{0.0265}{0.1600}$

0.0385

(

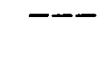

$--\quad \quad--$

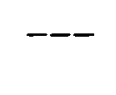




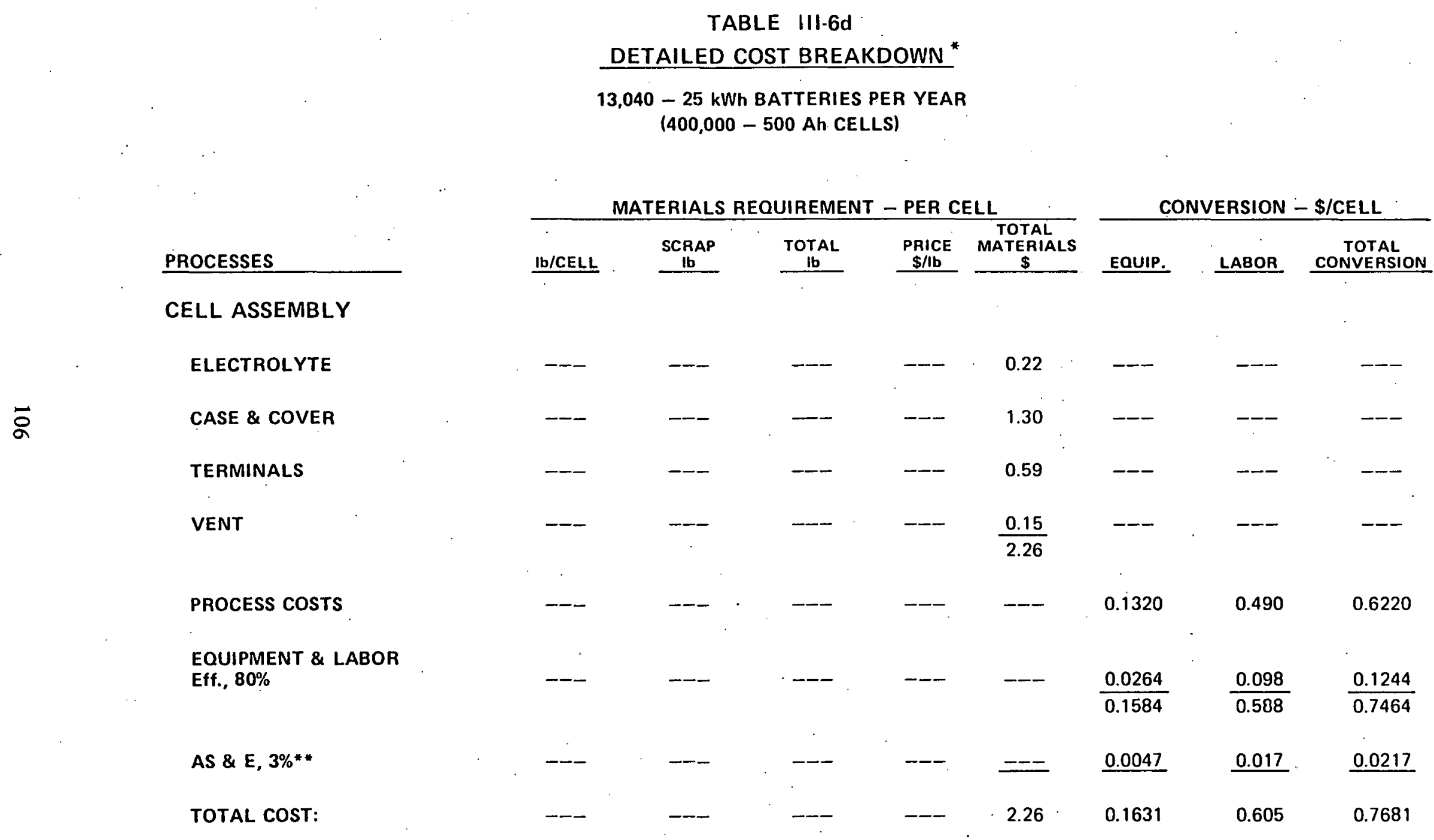

- See Appendix A, Partial Listing of Material Suppliers and Equipment Manufacturers.

* " Chosen arbitrarily at 3\%, actual will vary significantly depending on total Gould Ni-Zn battery business and the nature of industry which evolves. 
TABLE $\quad$ III-7

CAPITAL INVESTMENT ESTIMATES

(25 - kWh BATTERIES PER YEAR)

PRODUCTION RATE

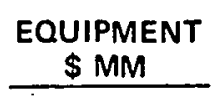

0.955

1.354

3.517

28,000 \begin{tabular}{c} 
FACILITY \\
\$MM \\
\hline
\end{tabular}

0.800

1.755

1.200

2.554

PRODUCTION

13,040

130,400
TOTAL

$\$ M M$
PILOT

6,520

(n)

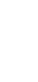

.


TABLE $\quad 111.8$

\section{CAPITAL EQUIPMENT BREAKDOWN}

\section{EQUIPMENT}

ELECTRODE PROCESSING

GENERAL EOUIPMENT:

TAB BLANK PRESS

DEGREASER

SHEAR

WELDER

BLENDER

ANNEALING FURNACE

NIO PROCESS EQUIPMENT:

MOLDING EQUIPMENT

CARRIERS

SINTERING FURNACE

ATMOSPHERE GENERATOR

GAS STORAGE

COIN \& BLANK PRESS

IMPREGNATION EQUIPMENT

Zn PROCESS EQUIPMENT:

HYDRAULIC PRESS

MOLDING EQUIPMENT

SEPARATOR MACHINE

ASSEMBLY EQUIPMENT

CONTINGENCY
PILOT FACILITY, \$MM

BATTERIES PER YEAR

1,304

6,520
PRODUCTION FACILITY, SMM

BATTERIES PER YEAR

13,040

130,400
0.103
0.103
0.168
0.620

0.562

0.771

2.174

18.380

0.115

0.230

0.575

5.500

0.025

0.050

0.100

0.800

0.050

0.100

0.300

2.400

0.100

0.100

TOTAL:

0.955

1.354

0.200

0.500

3.517

28.200 
TABLE $\quad 11 / .9$

MATERIALS REQUIREMENT PER YEAR *

MATERIALS - TONS PER YEAR

\begin{tabular}{|c|c|c|c|c|c|c|c|}
\hline \multirow[b]{2}{*}{$\begin{array}{l}\text { No. of } 25 \mathrm{kWh} \\
\text { BATTERIES/YEAR }\end{array}$} & \multicolumn{2}{|c|}{ Ni ELECTRODE } & \multicolumn{2}{|c|}{ Zn ELECTRODE } & \multirow[b]{2}{*}{$\begin{array}{c}\text { COPPER } \\
\text { (2) } \\
\end{array}$} & \multirow[b]{2}{*}{$\begin{array}{c}\text { PLASTIC CASE } \\
\& \text { COVER } \\
(3) \\
\end{array}$} & \multirow[b]{2}{*}{ SEPARATORS } \\
\hline & $\begin{array}{l}\text { NICKEL } \\
\text { METAL }\end{array}$ & $\begin{array}{c}\text { COBALT } \\
\text { METAL } \\
\text { (1) } \\
\end{array}$ & $\mathrm{ZnO}$ & BINDER & & & \\
\hline PILOT & & & & & & & \\
\hline 1,304 & 118 & 2.0 & 156.6 & 14.5 & 3.08 & 24.84 & 43.6 \\
\hline 6,520 & 590 & 10.0 & 783.0 & 72.5 & 15.4 & 124.2 & 218.0 \\
\hline \multicolumn{8}{|l|}{ PRODUCTION } \\
\hline 13,040 & 1,180 & 20.0 & 578.0 & 145.0 & 30.8 & 248.4 & 436.0 \\
\hline 130,400 & 11,800 & 200.0 & 5780.0 & 1450.0 & 308.0 & $2,484.0$ & $4,360.0$ \\
\hline
\end{tabular}

- See Appendix A, Partial Listing of Material Suppliers, and Equipment Manufacturers.

(1) Ni Tabs and Grids (Pos. and Neg.). Ni Powder (Pos. Substrate), Ni Plating For Cell Terminals and Connectors, and Positive Active Mass as Metallic Ni

(2) Ni Plated Copper Terminals and Connectors

(3) Calculated From Values on Gould's 400 Ah Polypropylene Case and Cover 


\begin{tabular}{|c|c|c|c|}
\hline & $\begin{array}{c}\text { REQUIREMENTS FOR } \\
130,400-25 \mathrm{kWh} \\
\text { BATTERIES/YEAR, } \\
\text { TONS }\end{array}$ & $\begin{array}{c}1975 \text { WORLD } \\
\text { PRODUCTION, } \\
\text { TONS } \\
\end{array}$ & $\begin{array}{l}\text { \% OF WORLD, } \\
\text { PRODUCTION }\end{array}$ \\
\hline NICKEL & 11,800 & $641,500^{(2)}$ & 1.84 \\
\hline ZINC & 4,624 & $5,519,000$ & 0.084 \\
\hline COPPER & 308 & $7,064,600$ & .004 \\
\hline COBALT & 200 & 30,900 & .65 \\
\hline POLYPROPYLENE & 2,624 & $1,300,000$ & .19 \\
\hline
\end{tabular}

(1) Metal Statistics, 1976.

(2) Nickel Reserve: 59.5 Million Tons (ore), Estimate From U.S. Bureau of Mines, 1976. 
NICKEL-ZINC BATTERY PROCESS DEMONSTRATION FACILITY

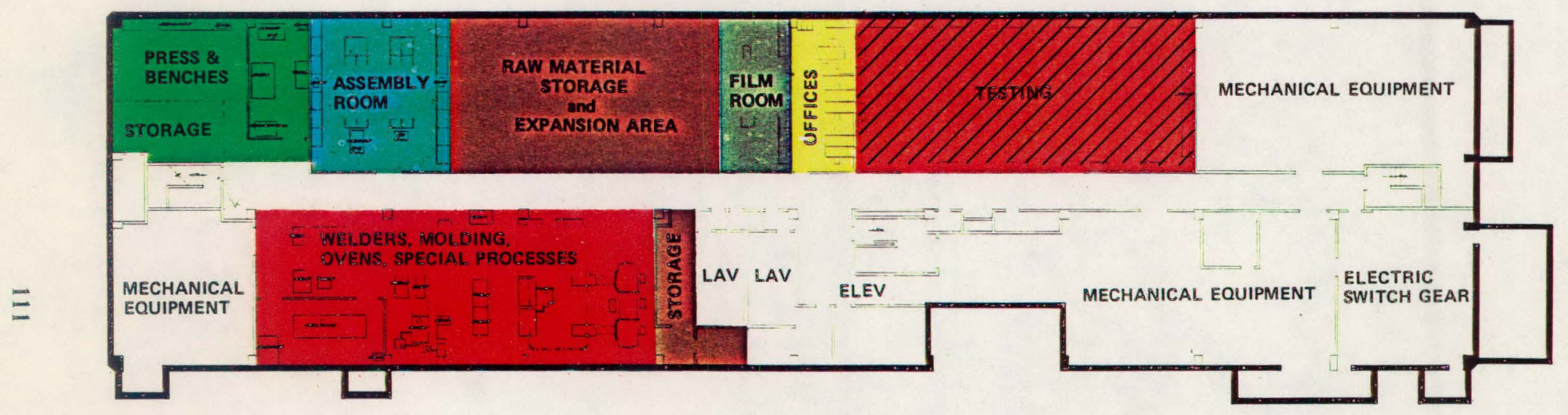

NICKEL ELECTRODE FAB.

ZINC ELECTRODE FAB.

CELL ASSEMBLY
Z2Д BAtTERY TEsting

SEPARATOR FABRICATION

PLANNED PROCESS EXPANSION \& STORAGE AREAS 
IV. PRELIMINARY TEST PROCEDURES, TEST STANDARDS, AND SAFETY

A. Test Procedures and Standards

1. General Requirements

a. Condition for Shipment - Batteries shall be shipped filled with electrolyte and fully discharged.

b. Markings - Batteries shall be marked with at least the following:

- Name of manufacturer

- Manufacturer's type designation

- Ah capacity and hour rating

- Month and year of manufacture

c. Instructions - Complete instructions for the maintenance of the battery, including charge and discharge characteristics, whall be included.

d. Terminal Markings Polarity - Polarity of positive and negative terminals shall be permanently marked "POS" or "+" and "NEG" or "-", respectively.

e. Posts and Intercell and Interbattery Connectors - Posts and connectors shall be adequate to carry, without excessive heating or voltage drop, the current incident to the service application. Copper components, when used, shall be protected against corrosion (e.g., nickel plated).

f. Cell Containers - Containers shall be of a plastic compound (e.g., polypropylene) and of a single cell or multi-cell construction.

g. Vent Caps - In the case of vented cells, a suitable baffling shall be provided to avoid spraying and to minimize creepage of electrolyte while at the same time permitting gas to escape. In the case of "sealed" or "maintenance-free" cells, a fail-safe device shall be provided to avoid excessive pressure build-up.

$h$. Seal - The joint between the cover and container, and all covered openings other than vents, shall be gas-tight and effectively sealed against creepage of electrolyte.

$i$. Impact Resistance of Containers - The containers shall be of the high impact type and, when tested in accordance with ASTM D-530, shall have an impact resistance of _ $*$ inch pounds min.

\section{Performance Requirements and Quality Assurance Provisions}

a. Capacity Ratings - Individual cells or batteries shall deliver a minimum of $90 \%$ of its rated 6-hour Ah capacity on or before the sixth cycle and a minimum of $100 \%$ on or before the ninth cycle. The cells or batteries shall deliver a minimum of $85 \%$ of their rated 6-hour capacity at the 1 -hour rate on or before the tenth cycle.

To determine conformance with the above, the cells and batteries shall be subjected to the following test cycles:

- Cycles 1 to 3 (conditioning cycles) - Discharge at the 6 -hour rate at $77^{\circ} \mathrm{F},(+10,-5)$ to an end voltage of 1.30 volts per cell after charging in accordance with the instructions furnished by the manufacturer.

* to be determined 
- Cycles 4 to 6 - Charge at the 6 to 10-hour rate. The total charge shall not exceed $110 \%$ of its rated 6 -hour capacity.

Discharge at the 6-hour rate at $77^{\circ} \mathrm{F}(+10,-5)$ to an end voltage of 1.30 volts per cell to determine capacity (minimum $90 \%$ of rated on sixth cycle). Constant current or constant potential charging instructions shall be furnished by the manufacturer.

- Cycles 7 to 9 - Charge at the 6 to 10-hour rate. The total charge shall not exceed $110 \%$ of its rated 6 -hour capacity. Discharge at the 6 -hour rate at $77^{\circ} \mathrm{F}(+10,-5)$ to an end voltage of 1.30 volts per cell to determine full capacity (minimum of $100 \%$ of rated on ninth cycle.).

- Cycle 10 - Charge at the 6 to 10 -hour rate. The total charge shall not exceed $110 \%$ of its rated 6-hour capacity.

Discharge at the 1 -hour rate $\left(0.85 \times 6\right.$-hour rated capacity in amperes) at $77^{\circ} \mathrm{F}$ $(+10,-5)$ to an end voltage of 1.15 volts per cell to determine capacity (minimum $85 \%$ of 6 -hour rated capacity).

b. Retention of Charge - Cells or batteries shall not lose in excess of $20 \%$ of rated capacity in 14 days when tested as described below:

Cells or batteries shall be charged in an ambient temperature of $72^{\circ}$ to $87^{\circ} \mathrm{F}$, then allowed to stand on open circuit in an ambient temperature of $72^{\circ}$ to $87^{\circ} \mathrm{F}$ for a period of 14 days, and discharged at the 6-hour rate to 1.30 volts per cell.

c. Cycle Life - A battery shall deliver at least 500 cycles to $80 \%$ of its rated 6-hour capacity or at least 1000 cycles to $80 \%$ of its rated 6-hour capacity when tested as described below:

- 500 Cycle Test - Discharge for 4 hours at a rate equivalent to removing $80 \%$ of the 6-hour capacity rating. Recharge in 8 hours. The total charge shall not exceed $110 \%$ of the capacity discharged. One complete capacity test at the 6-hour rate shall be made once a month. Ex. for 500 Ah cell: $400 \mathrm{Ah}$ out $(80 \%)$ at $100 \mathrm{~A}$ for 4 hours $400 \mathrm{Ah}$ in $(110 \%)$ at $55 \mathrm{~A}$ for 8 hours

- 1000 Cycle Test - Discharge for 2.5 hours at a rate equivalent to removing $50 \%$ of the 6-hour capacity rating. Recharge in 5 hours. The total charge shall not exceed $110 \%$ of the capacity discharged. One complete capacity test at the 6-hour rate shall be made once a month. Ex. for 500 Ah capacity:

$250 \mathrm{Ah}$ out $(50 \%)$ at $100 \mathrm{~A}$ for 2.5 hours

$275 \mathrm{Ah}$ in $(110 \%)$ at $55 \mathrm{~A}$ for 5.0 hours

The temperature shall be maintained between $72^{\circ}$ and $87^{\circ} \mathrm{F}$ throughout the test.

\section{Test Equipment}

All equipment shall be clean, in good working order, and calibrated. The conditions described in NEMA Standards Publication Nr 1B2-1974, Section 1B2-1.03, Part H shall apply. 


\section{Pre and Post Test Examination Procedures}

a. Pre-Test Inspection - The cell or battery shall undergo visual inspection for defects and conformance to an acceptable quality level. The following shall be included in the inspection:

- Electrolyte leaks at the joint between the cover and container and terminals

- Mechanical defects such as loose posts and connectors, legibilty of terminal marking polarities, container cracks, and loose vent plugs

The open circuit voltage shall be measured to check for possible shorts. Following the third conditioning charge, the cell or battery shall be allowed to stand on open circuit for a minimum of 24 hours and the voltage shall equal at least $\ldots$ volts per cell.

b. Post-Test Examination - The cell or battery shall undergo the visual inspection for defects as outlined in the Pre-Test Inspection.

Each component of the cell shall be examined and the findings recorded. Where appropriate, physical analytical methods shall be used to determine the conditions of the components. The methods of analysis for the separator and electrodes would consist of the following:

- Separator - Physical structure determination,e.g., pore size, pore size distribution, etc., high resolution scanning electron microscopy, qualitative and quantitative presence of elements by electron microprobe techniques, elemental x-ray mapping to determine location of elements, infra-red spectroscopy to determine the chemical degradation mechanism(s), and $x$-ray photoelectron spectrometer for analyzing the oxidation state of the elements detected in the separator.

- Electrodes - General physical appearance of the electrode, alteration in morphology and microstructure (surface) using scanning electron microscopy, and x-ray diffraction techniques, surface compositional and oxidation state of analysis employing auger electron spectroscopy and $\mathrm{x}$-ray photoelectron spectroscopy.

\section{B. Special Safety Considerations}

Proper ventilation of gases is necessary to safeguard against possible hazards (explosion). No sparks, flames, or the like should be permitted near the battery compartment during charge.

Battery maintenance (e.g., water addition, connector servicing, etc.) should not be done while battery is in operation.

Precautions should be taken against leaving metal tools in the area of the connectors, causing accidental shorts.

Explosion-proof vents should be used.

Precautions should be taken in operating a battery with partially shorted cells ("hot spot" which could create a fire hazard) or loose connectors resulting in localized high resistance.

*to be determined 


\section{REFERENCES}

1. Port, F.J., "Energy Sources”, Fourth International Electric Vehicle Symposium, Dusseldorf, Germany, August 31, 1976.

2. Sheibley, D.W., "Separators for Nickel-Zinc Batteries", The Electrochemistry Society 149th Meeting, Washington, D.C., May 5, 1976.

3. Charkey, A., "Development of Large Size Nickel-Zinc Cells for Electric Vehicles", Fourth International Electric Vehicle Symposium, Dusseldorf, Germany, Sept. 1, 1976.

4. Charkey, A., "Advances in Component Technology for Nickel-Zinc Cells for Electric Vehicles", Eleventh Intersociety Conversion Engineering Conference, State Line, Nevada, September 14, 1976.

5. Yew, M.C. and McCulloch, D. E., "Small Electric Vehicle Considerations in View of Performance and Energy Usage", Eleventh Intersociety Energy Conversion Engineering Conference, State Line, Nevada, September 13, 1976. 


\title{
APPENDIX A \\ PARTIAL LISTING OF MATERIALS SUPPLIERS AND EQUIPMENT MANUFACTURERS
}

The prices shown in the manufacturing cost breakdown for the key materials were obtained recently from our supplicrs. Costs of the various equipment are from actual quotations. Some of the organizations contacted are listed below.

\author{
International Nickel Company, \\ New York \\ The New Jersey Zinc Company, \\ Bethlehem, Pennsylvania \\ Dupont, \\ Wilmington, Delaware \\ BTU Engineering Corporation, \\ Massachusetts \\ Littleford Brothers, Inc., \\ Cincinnati, Ohio \\ Pfizer Metals \& Composit Products \\ Wallingford, Connecticut \\ Anderson Crane Company, \\ Minneapolis, Minnesota \\ Richards - Wilcox \\ Aurora, Illinois \\ Abar Corporation, \\ Feasterville, Pennsylvania \\ Brew, Inc., \\ Concord, New Hampshire \\ Bushman Equipment Company, \\ Milwaukee, Wisconsin
}


Air Technical Industries Mentor, Ohio

Drever Company

Huntingdon, Pennsylvania

GCA/Vacuum Industries

Somerville, Massachusetts

WSF Industries, Inc.,

Tonawanda, New York

Alpine American Corporation, Natick, Massachusetts

Sweco, Inc.,

Florence, Kentucky

Sturtevant Mill Company,

Boston, Massachusetts

Airco Speer Carbon - Graphite

St. Mary's, Pennsylvania

Union Carbide Corporation, Chicago, Illinois

Becker Brothers Carbon Corporation, Cicero, Illinois

Patterson - Kelly Company

East Stroudsburg, Pennsylvania

Blue M Electric Company, Blue Island, Illinois

Grieve Corporation

Round Lake, Illinois

Pasadena Presses

City of Industry, California

Rucker PHI,

Brea, California

Dairy .Craft, Inc.,

St. Cloud, Minnesota 
Peter D. Warner Company, Minneapolis, Minnesota

Edelmann \& Associates, Inc., Minneapolis, Minnesota

Goodin Company,

St. Paul, Minnesota

Vibra-Screw,

Totawa, New Jersey

Systems Engineering \& Manufacturing Company, Houston, Texas 
Peter D. Warner Company, Minneapolis, Minnesota

Edelmann \& Associates, Inc., Minneapolis, Minnesota

Goodin Company,

St. Paul, Minnesota

Vibra-Screw,

Totawa, New Jersey

Systems Engineering \& Manufacturing Company, Houston, Texas 